\title{
Isoperimetric inequalities in Riemann surfaces of infinite type
}

\author{
Venancio Alvarez, Domingo Pestana and José M. Rodríguez
}

\section{Introduction.}

By $\mathcal{S}$ we denote a hyperbolic Riemann surface, i.e. a (open and connected) Riemann surface whose universal covering space is the unit disk $\mathbb{D}=\{z \in \mathbb{C}:|z|<1\}$, endowed with its Poincaré metric (also called the hyperbolic metric), i.e. the metric obtained by projecting the Poincaré metric of the unit disk

$$
d s=\frac{2|d z|}{1-|z|^{2}} .
$$

With this metric, $\mathcal{S}$ is a complete Riemannian manifold with constant curvature -1 . The only Riemann surfaces which are left out are the sphere, the plane, the punctured plane and the tori.

It is convenient to remark that this definition of hyperbolic Riemann surface is not universally accepted, since sometimes the word hyperbolic refers to the existence of Green's function.

We say that $\mathcal{S}$ satisfies the hyperbolic isoperimetric inequality (HII) if $\mathcal{S}$ is a hyperbolic Riemann surface and there exists a constant $h>0$ such that for every relatively compact domain (an open and connected set) $G$ with smooth boundary one has that

$$
A_{\mathcal{S}}(G) \leq h L_{\mathcal{S}}(\partial G)
$$

where $A_{\mathcal{S}}(G)$ denotes the (hyperbolic) area of $G$ and $L_{\mathcal{S}}(\partial G)$ the (hyperbolic) length of its boundary. An approximation argument gives 
that if $\mathcal{S}$ satisfies HII, then (1.1) is also true for domains with finite area. We denote by $h(\mathcal{S})$ the best constant in $(1.1)$

It is clear that a finite area hyperbolic Riemann surface does not satisfy HII.

A Riemann surface $\mathcal{S}$ is said to be of finite type if its fundamental group $\Pi_{1}(p, \mathcal{S}), p \in \mathcal{S}$, is finitely generated. In other case we say that $\mathcal{S}$ is of infinite type. It is well known that every Riemann surface of finite type can be obtained from a compact Riemann surface by deleting $p$ points (the punctures of $\mathcal{S}$ ) and $n$ closed disks (whose boundaries represent the ideal boundaries of $\mathcal{S}$ ). It is also a known fact that a Riemann surface of finite type has HII if and only if $n>0$ or, equivalently, if $\mathcal{S}$ has infinite area. Therefore, in spite of most of our results are true independently of the type of the considered Riemann surface, we will be interested in Riemann surfaces of infinite type.

There are a number of natural questions concerning the HII-property of Riemann surfaces. Particularly interesting are the stability under quasiconformal maps, its relation with other conformal invariants and its characterization for plane domains. Here the word conformal refers to holomorphic homeomorphisms.

Concerning the study of the stability of HII, in [FR, Theorem 1] it was proved that if two Riemann surfaces are quasiconformally equivalent and one has HII, the other has too.

One of the conformal invariants related with the HII-property is the bottom of the spectrum of the Laplace-Beltrami operator, $b(\mathcal{S})$, which can be defined in terms of Rayleigh's quotients as

$$
b(\mathcal{S})=\inf _{\varphi \in C_{c}^{\infty}(\mathcal{S})} \frac{\iint\|\nabla \varphi\|^{2} d w}{\iint \varphi^{2} d w},
$$

where $\|\cdot\|, \nabla$ and $d w$ refer to the Poincaré metric of $\mathcal{S}$.

The number $b(\mathcal{S})$ belongs to $[0,1 / 4]$ and a celebrated theorem of Elstrodt, Patterson and Sullivan [Su, p. 333] relates it with other important conformal invariant of $\mathcal{S}$, its exponent of convergence $\delta(\mathcal{S})$ (see e.g. [N, p. 21] for basic background), which can be defined as

$$
\delta(\mathcal{S}):=\inf \left\{t: U_{t}(p)<\infty, \text { for some } p \in \mathcal{S}\right\},
$$

where

$$
U_{t}(p):=\sum_{[\gamma] \in \Pi_{1}(p, \mathcal{S})} \exp \left(-t L_{\mathcal{S}}([\gamma])\right)
$$


and

$$
L_{\mathcal{S}}([\gamma]):=\inf \left\{L_{\mathcal{S}}(g):[\gamma]=[g]\right\} .
$$

It is easy to check that if $U_{t}(p)<\infty$ for some $p \in \mathcal{S}$, then $U_{t}(q)<\infty$ for all $q \in \mathcal{S}$.

It is a well known fact that $0 \leq \delta(\mathcal{S}) \leq 1$ (

The theorem of Elstrodt, Patterson and Sullivan asserts that

$$
b(\mathcal{S})= \begin{cases}\frac{1}{4}, & \text { if } 0 \leq \delta(\mathcal{S}) \leq \frac{1}{2} \\ \delta(\mathcal{S})(1-\delta(\mathcal{S})), & \text { if } \frac{1}{2} \leq \delta(\mathcal{S}) \leq 1\end{cases}
$$

It is also well known (see e.g., [Ch1, p. 95], [Che], [FR, Theorem 2]) that

$$
\frac{1}{4} \leq b(\mathcal{S}) h(\mathcal{S})^{2} \quad \text { and } \quad b(\mathcal{S}) h(\mathcal{S}) \leq C<\frac{3}{2},
$$

where $C$ is an absolute constant.

Therefore $\mathcal{S}$ has the HII-property if and only if $b(\mathcal{S})>0$ or, equivalently, $\delta(\mathcal{S})<1$.

A theorem of Myrberg [T, p. 522] states that if $\delta(\mathcal{S})<1$ then $\mathcal{S}$ has Green's function, or equivalently, that it possesses non-constant positive superharmonic functions (see [AS, p. 204] or [T, p. 434]). Therefore if $\mathcal{S}$ has finite genus, $\mathcal{S}$ has non-constant harmonic functions with finite Dirichlet integral [AS, p. 208], [SN, p. 332]. In the general case, the conclusion is also true with additional hypothesis [Ro1]. However, there exists a Riemann surface $\mathcal{S}_{0}$ having infinite genus and HII such that the constants are the unique positive harmonic functions in $\mathcal{S}_{0}$ [Ro2]. Recall that if there exists a non-constant harmonic function with finite Dirichlet integral, then there exists a non-constant positive (in fact, bounded) harmonic function.

It is also known that $\delta(\mathcal{S})$ coincides with the Hausdorff dimension of the conical limit set of the covering group of $\mathcal{S}$ (see e.g. [N, p. 154]). This says us that the HII-property must be also related with the size of the "boundary" of $\mathcal{S}$.

At the moment no characterization of the HII-property is known for hyperbolic plane domains (i.e. subsets of the Riemann sphere whose boundary has at least three points) in euclidean terms of the size of its boundary. In [FR, Theorems 3 and 4] a sufficient condition and a necessary condition were obtained so that a hyperbolic plane domain satisfies HII, but none of them constitutes a characterization of the HII-property, although these conditions are quite close. 
As an example of the difficulties involving the problem, recall that a plane domain $\Omega$ has Green function if and only if its boundary has positive logarithmic capacity (see [AS, p. 249], [T, p. 440] or [SN, p. 332-333]). But, for example, $\mathbb{D} \backslash\{0\} \backslash\left\{1 / 2^{n}\right\}_{n=1}^{\infty}$ has not HII, while $\mathbb{D} \backslash\left\{1-1 / 2^{n}\right\}_{n=0}^{\infty}$ has it (these facts are consequence of [FR, Theorems 3 and 4] or Theorem 1 below). Hence, this shows that the problem of deciding whether a hyperbolic plane domain has the HII-property or not is delicate. Observe that if $\Omega$ is a hyperbolic plane domain and $\partial \Omega$ has zero logarithmic capacity, then $\Omega$ has not HII.

The main results of this paper are Theorems 1, 3, 5, 9 and 10. Theorem 1 shows that for any hyperbolic Riemann surface the HII-property is preserved by removing a sufficiently separated set. Theorem 3 relates simple euclidean conditions with the HII-property in Denjoy domains. Theorem 5 gives an euclidean characterization of Denjoy domains satisfying the HII-property. Finally, Theorems 9 and 10 give localization results for the HII-property in general planar domains.

In the next section we give some definitions needed to state our results.

\section{The main results.}

We say that a domain $G \subset \hat{\mathbb{C}}$ is modulated if there is an upper bound for the modulus of every doubly connected domain contained in $G$ which separates the boundary of $G$. In particular, every simply connected domain is modulated (since in this case there are not such doubly connected domains). Also, if the boundary of $G$ consists of a finite number of continua, $G$ is modulated. On the other hand, if the boundary of $G$ has an isolated point, $G$ is not modulated.

These are the domains in the plane that as far as Function Theory is concerned behave almost like simply connected domains (see for example $[\mathrm{BP}]$ and the references therein).

In $[\mathrm{FR}$, Theorem 3] it was proved that if $G \subset \hat{\mathbb{C}}$ is modulated (and therefore $G$ has HII) then $H=G \backslash\left\{a_{n}\right\}$ has also HII if the sequence $\left\{a_{n}\right\}$ is uniformly separated in the hyperbolic metric of $G$, i.e. if there exists a positive constant $c$ such that

$$
d_{G}\left(a_{n}, a_{m}\right)>c, \quad \text { for all } n \neq m
$$

where $d_{G}$ denotes the hyperbolic distance in $G$. This result is not true if $G$ is not modulated (see Theorem 1 below). Obviously, every finite 
sequence is uniformly separated, and a sequence converging to a point of $G$ is not uniformly separated.

Conversely, also in [FR, Theorem 4], it was proved that if $H \subset \hat{\mathbb{C}}$ has HII, and $G=H \cup I$, where $I$ is the set of isolated points of $\partial H$, then $I$ is uniformly separated in the hyperbolic metric of $G$.

In this work we reduce the study of the HII-property of $H$ to that of $G$, not only for hyperbolic plane domains, but for general hyperbolic Riemann surfaces.

To state our result, we need a previous definition.

Definition. A subset I of a hyperbolic Riemann surface $\mathcal{S}$ is strongly uniformly separated in $\mathcal{S}$, if there exists a positive constant $r_{0}$ such that the hyperbolic balls $B_{\mathcal{S}}\left(p, r_{0}\right)$, where $p \in I$, are simply connected and pairwise disjoint.

Theorem 1. Let $\mathcal{S}$ be a hyperbolic Riemann surface, let I be a closed and countable subset of $\mathcal{S}$ and $\mathcal{R}=\mathcal{S} \backslash I$. Then, $\mathcal{R}$ has HII if and only if $\mathcal{S}$ has HII and $I$ is strongly uniformly separated in $\mathcal{S}$.

We also have obtained a relationship between the isoperimetric constants on $\mathcal{R}$ and $\mathcal{S}$ (see Section 3 below).

We want to remark that Theorem 1 is a new result even in the case of plane domains.

Corollary 1. Let $\mathcal{S}$ be a hyperbolic Riemann surface, let I be a closed and countable subset of $\mathcal{S}$ and let $\mathcal{R}=\mathcal{S} \backslash I$. If I has an accumulation point in $\mathcal{S}$, then $\mathcal{R}$ has not HII.

Observe that Theorem 1 and [FR, Theorem 3] give that every discrete set which is uniformly separated in a modulated domain $G$ is also strongly uniformly separated in $G$.

As we mentioned above, at the moment no characterization of the HII-property is known for hyperbolic plane domains in euclidean terms of the size of its boundary. In [FR] it was obtained a necessary condition and a sufficient condition so that a hyperbolic plane domain has HII, but we know that none of them is, in fact, a characterization of the HIIproperty for this type of Riemann surfaces. In this paper we obtain a characterization of the HII-property for the case of Denjoy domains, i.e. hyperbolic plane domains whose boundary is contained in $\hat{\mathbb{R}}$, in euclidean terms of the size of their boundaries. 
Since the HII-property is a quasiconformal invariant between general Riemann surfaces [FR, Theorem 1] our results characterize the HIIproperty for subsets of $\hat{\mathbb{C}}$ whose boundary is contained in a quasicircle. In fact we can prove a more general result (see Section 7).

Definition. Let $\Omega$ be a plane domain, let $I$ be the set of isolated points of $\partial \Omega$ and $\Omega_{0}=\Omega \cup I$. We say that $\Omega$ is admissible if $\Omega_{0}$ is a hyperbolic plane domain and $I$ is strongly uniformly separated in $\Omega_{0}$.

Observe that if $\Omega$ is admissible, then there are no isolated points in $\partial \Omega_{0}$; therefore $\partial \Omega_{0}$ has infinitely many points and $\Omega$ has infinite area.

Now we can restate Corollary 1 for hyperbolic plane domains.

Corollary 2. If a hyperbolic plane domain is not admissible, then it has not HII.

In what follows $\Omega \subset \hat{\mathbb{C}}$ will usually be a Denjoy domain. In order to establish our characterization of the HII-property for Denjoy domains (Theorem 5) we need some preliminary background.

For $\alpha<\beta,(\beta, \alpha)$ denotes the set $\{x \in \mathbb{R}: x<\alpha$ or $x>\beta\} \cup\{\infty\}$. Also we mean that $(\infty, \alpha)=\{x \in \mathbb{R}: x<\alpha\}$ and as usual $(\alpha, \infty)=$ $\{x \in \mathbb{R}: x>\alpha\}$. Along the paper we mean that the point at infinity is the greatest of the numbers in $\hat{\mathbb{R}}$.

Definition. We say that a finite subset $A=\left\{a_{1}, \ldots, a_{2 n}\right\}(n \geq 2)$ of points of $\partial \Omega \subset \hat{\mathbb{R}}$ is a border set of $\partial \Omega$ if $A$ verifies the following two conditions:

i) $A$ is "ordered" in $\hat{\mathbb{R}}$, i.e. there exists $j \in \mathbb{Z}_{2 n}=\mathbb{Z} /(2 n \mathbb{Z})$ such that $a_{j+1}<\cdots<a_{j+2 n}$, where the subscripts belong to $\mathbb{Z}_{2 n}$.

ii) The open set $\cup_{k=1}^{n}\left(a_{2 k-1}, a_{2 k}\right)$ is contained in $\Omega$.

Obviously every finite subset $A=\left\{a_{1}, \ldots, a_{2 n}\right\}$ of $\hat{\mathbb{R}}$ can be "ordered" in such a way that the condition i) is satisfied. So ii) is the significant condition in the definition above.

ExAmple. Let us consider the Denjoy domain $\Omega$ whose boundary is $\partial \Omega=\{\infty\} \cup\left(\cup_{n=1}^{\infty}[2 n-1,2 n]\right)$. It is clear that the ordered sets $\{2,3,6,7,10,11\}$ and $\{4,5, \infty, 1\}$ are border sets of $\partial \Omega$, but $\{1,4,5, \infty\}$ is not. In fact, the ordered set of real numbers $\left\{a_{1}, \ldots, a_{2 n}\right\}$ is a border set if and only if $a_{2 k}=1+a_{2 k-1}$ and $a_{2 k-1} \in 2 \mathbb{Z}$ for $k=1, \ldots, n$. On 
the other hand, the ordered set $\left\{a_{1}, \ldots, a_{2 n-1}, \infty\right\}$ never is a border set. The ordered set $\left\{a_{1}, \ldots, a_{2 n-2}, \infty, a_{2 n}\right\}$, with $n \geq 3$ and $a_{1}<$ $\cdots<a_{2 n-2}$ is a border set if and only if $\left\{a_{1}, \ldots, a_{2 n-2}\right\}$ is a border set and $a_{2 n}=1$.

Observe that the set of four "consecutive points", $\left\{a_{2 k-1}, a_{2 k}\right.$, $\left.a_{2 k+1}, a_{2 k+2}\right\}$ with $k \in \mathbb{Z}_{2 n}$, of a border set of $\Omega$ is also a border set of $\Omega$. Besides, observe that if $\partial \Omega$ has not any border set, then $\Omega$ is some of the three following trivial domains (up conformal equivalence): $\mathbb{C} \backslash\{0,1\}$ (which has not HII), $\mathbb{C} \backslash[0,1]$ (which has HII), $\hat{\mathbb{C}} \backslash[0,1]$ (which has HII).

If $B=\left\{b_{1}, b_{2}, b_{3}, b_{4}\right\}$, we denote by $r(B)$ the cross ratio

$$
r(B)=\frac{\left(b_{2}-b_{1}\right)\left(b_{4}-b_{3}\right)}{\left(b_{3}-b_{2}\right)\left(b_{4}-b_{1}\right)} .
$$

In the following by $\Phi_{1}, \Phi_{2}:(0, \infty) \longrightarrow(0, \infty)$ we denote any fixed continuous functions satisfying the following properties:

a) $\Phi_{1}(r) \asymp \Phi_{2}(r) \asymp \frac{1}{\log r}$ as $r \longrightarrow \infty$,

b) $\Phi_{1}(r) \asymp \log \frac{1}{r}$ and $\Phi_{2}(r) \asymp \log \log \frac{1}{r}$ as $r \longrightarrow 0$.

After these preliminaries we can state the following partial result which gives a necessary condition and a sufficient condition for the HIIproperty of a Denjoy domain $\Omega$. In many cases these conditions give an answer to the question of whether or not $\Omega$ has HII, since they are very close.

Theorem 3. Let $\Omega$ be an admissible Denjoy domain, let $I$ be the set of isolated points of $\partial \Omega$ and $\Omega_{0}=\Omega \cup I$.

a) If $\Omega$ has HII, then there exists a positive constant $c$ such that for any border set of $\partial \Omega_{0}, B=\left\{b_{1}, \ldots, b_{2 n}\right\}$ with $n \geq 3$, we have that

$$
\frac{1}{n} \sum_{j=1}^{n} \Phi_{1}\left(r\left(\left\{b_{2 j-1}, b_{2 j}, b_{2 j+1}, b_{2 j+2}\right\}\right)\right)>c .
$$

b) If there exists a positive constant $c$ such that for any border set of $\partial \Omega_{0}, B=\left\{b_{1}, \ldots, b_{2 n}\right\}$ with $n \geq 3$, we have that

$$
\frac{1}{n} \sum_{j=1}^{n} \Phi_{2}\left(r\left(\left\{b_{2 j-1}, b_{2 j}, b_{2 j+1}, b_{2 j+2}\right\}\right)\right)>c,
$$


then $\Omega$ has HII.

Besides, we have a characterization of the Denjoy domains with HII in euclidean terms of the size of their boundaries. This characterization has the disadvantage that the function which appears instead of $\Phi_{1}$ and $\Phi_{2}$ (in Theorem 3) is more complicated and depends on the domain.

Theorem 5. Let $\Omega$ be a Denjoy domain, let I be the set of isolated points of $\partial \Omega$ and let $\Omega_{0}=\Omega \cup I$. Then, $\Omega$ has HII if and only if $\Omega$ is admissible and there exists a positive constant $c$ such that for any border set of $\partial \Omega_{0}, B=\left\{b_{1}, \ldots, b_{2 n}\right\}$ with $n \geq 3$, we have that

$$
\frac{1}{n} \sum_{j=1}^{n} \Psi_{\Omega_{0}}\left(\left\{b_{2 j-1}, b_{2 j}, b_{2 j+1}, b_{2 j+2}\right\}\right)>c,
$$

where $\Psi_{\Omega_{0}}$ is the function appearing in Theorem 4 (see Section 5).

Roughly speaking, this function $\Psi_{\Omega_{0}}$ "counts" in some sense the number of annuli which intersect $\partial \Omega_{0}$.

If $\mathcal{S}_{2}$ is a hyperbolic Riemann surface, we will consider (open and connected) subsurfaces $\mathcal{S}_{1} \subset \mathcal{S}_{2}$, endowed with its own hyperbolic metric (recall that any subsurface of a hyperbolic Riemann surface is also hyperbolic). Of course, with this metric $\mathcal{S}_{1}$ is a complete Riemannian manifold.

As a direct consequence of Corollary 7 (see Section 7 below) we obtain two localization theorems.

Theorem 9. Given a closed subset $E$ of $\hat{\mathbb{C}}$ with infinitely many points, the following conditions are equivalent:

1) $\hat{\mathbb{C}} \backslash E$ satisfies HII.

2) $\Omega \backslash E$ satisfies HII, for any subdomain $\Omega$ of $\hat{\mathbb{C}}$ of finite type such that $E$ is contained in $\Omega$.

3) $\Omega \backslash E$ satisfies HII, for some subdomain $\Omega$ of $\hat{\mathbb{C}}$ of finite type such that $E$ is contained in $\Omega$.

Theorem 10. Let $E_{1}, \ldots, E_{n}$ be pairwise disjoint closed subsets in $\hat{\mathbb{C}}$ with infinitely many points such that $\Omega=\hat{\mathbb{C}} \backslash \cup_{k} E_{k}$ is connected. Then, we have that $\Omega$ satisfies HII if and only if $\hat{\mathbb{C}} \backslash E_{k}$ satisfies HII for $k=1, \ldots, n$. 
In fact, we prove in Section 7 a general version of theorems 9 and 10 about Riemann surfaces (see Theorem 7). We should remark that we have also obtained other results on localization (see for example Lemmas 3.1 and 7.1 or Corollary 5).

\subsection{Notations and background.}

As usual, $\mathbb{R}$ and $\hat{\mathbb{R}}$ will denote the real line and the extended real line. Similarly, $\mathbb{C}$ and $\hat{\mathbb{C}}$ will denote, respectively, the complex plane and the Riemann sphere. The simbol $A \backslash B$ denotes the difference of the sets $A$ and $B$. The expression $A(r) \asymp B(r)$ will mean that there exists a positive constant $C$ such that

$$
C^{-1} \leq \frac{A(r)}{B(r)} \leq C,
$$

for the values of $r$ indicated in each case. We denote by $[x]$ the greatest natural number which is less or equal than $x$.

By $d_{\mathcal{S}}$ and $B_{\mathcal{S}}$ we shall denote, respectively, the distance and the balls in the Poincaré metric of $\mathcal{S}$. By $d$ and $B$ we shall denote, respectively, the distance and the balls in the euclidean metric of $\mathbb{C}$. $B_{\mathcal{S}}^{*}$ and $B^{*}$ will denote the corresponding balls without its centers. If $\Omega$ is a hyperbolic plane domain, $\delta_{\Omega}(z)$ will be the euclidean distance of $z$ to the boundary of $\Omega$. By $\lambda_{\Omega}$ we shall denote the conformal density of the Poincaré metric in $\Omega$, i.e. the function such that $d s=\lambda_{\Omega}(z)|d z|$ is the Poincaré metric in $\Omega$. For $\alpha<\beta,(\beta, \alpha)$ denotes the set $\{x \in \mathbb{R}: x<\alpha$ or $x>\beta\} \cup\{\infty\}$. Also we mean that $(\infty, \alpha)=\{x \in \mathbb{R}: x<\alpha\}$ and as usual $(\alpha, \infty)=\{x \in \mathbb{R}: x>\alpha\}$. We define the corresponding closed intervals in a similar way. Along the paper we mean that the point at infinity is the greatest of the numbers in $\hat{\mathbb{R}}$.

Finally, we denote by $c$ positive constants which can assume different values from line to line and even in the same line. On the other hand, the constants $t_{0}$ and $r_{0}$ will have always the same value.

In order to prove our results we shall need some well known facts concerning the Poincaré metric:

1) A conformal map between two hyperbolic Riemann surfaces is an isometry.

2) If $\mathcal{S}_{1}$ is a subsurface of the hyperbolic Riemann surface $\mathcal{S}_{2}$, then $d_{\mathcal{S}_{1}}(p, q) \geq d_{\mathcal{S}_{2}}(p, q)$, for all $p, q \in \mathcal{S}_{1}$. 
3) Let $\mathcal{S}_{1}$ be a subsurface of the hyperbolic Riemann surface $\mathcal{S}_{2}$ and let $\sigma$ be a simple closed curve in $\mathcal{S}_{1}$. Denote by $\gamma_{j}$ the simple closed geodesic (if exists) freely homotopic to $\sigma$ in $\mathcal{S}_{j}(j=1,2)$. Then $L_{\mathcal{S}_{1}}\left(\gamma_{1}\right) \geq L_{\mathcal{S}_{2}}\left(\gamma_{2}\right)$.

4) If $\Omega$ is a hyperbolic plane domain, $\Omega \subset \mathbb{C}$, then $\lambda_{\Omega}(z) \leq 2 / \delta_{\Omega}(z)$, for all $z \in \Omega$ (recall that $\delta_{\Omega}(z)$ denotes the euclidean distance of $z$ to the boundary of $\Omega$ ).

5) A hyperbolic plane domain $\Omega, \Omega \subset \mathbb{C}$, is modulated if and only $\lambda_{\Omega}(z) \delta_{\Omega}(z) \asymp 1$, for $z \in \Omega$ (see [BP, Corollary 1]). The constant in $\asymp$ depends on $\Omega$.

6) For $\Omega \subset \mathbb{C}$, define $\beta_{\Omega}(z)$ as the function

$$
\beta_{\Omega}(z)=\inf \left\{|\log | \frac{z-a}{b-a}||: a, b \in \partial \Omega,|z-a|=\delta_{\Omega}(z)\right\} .
$$

In $[\mathrm{BP}$, Theorem 1] it was proved that

$$
\lambda_{\Omega}(z) \delta_{\Omega}(z)\left(1+\beta_{\Omega}(z)\right) \asymp 1, \quad \text { for } z \in \Omega,
$$

up to universal constants. See (6.1) below for a precise estimate.

7) If $F: \mathbb{D} \longrightarrow \Omega$ is a universal covering map, then we have

$$
\lambda_{\Omega}(F(z))\left|F^{\prime}(z)\right|=\lambda_{\mathbb{D}}(z), \quad \text { for all } z \in \mathbb{D} .
$$

The organization of the paper is as follows. In sections 3 and 4 we prove, respectively, theorems 1 and 3 . Theorems 4 and 5 will be proved in Section 5. Section 6 contains a proposition relating balls and collars of punctures. In Section 7 we develop some useful technology to prove theorems 9 and 10 and other further results. In Section 8 we discuss the relationship between the HII-property, polarization and circular symmetrization. Finally we discuss about the possibility to improve Theorem 5 in sections 9 and 10.

\section{Proof of Theorem 1.}

Theorem 1. Let $\mathcal{S}$ be a hyperbolic Riemann surface, let $I$ be a closed and countable subset of $\mathcal{S}$ and $\mathcal{R}=\mathcal{S} \backslash I$. Then, $\mathcal{R}$ has HII if and only if $\mathcal{S}$ has HII and $I$ is strongly uniformly separated in $\mathcal{S}$. 
More precisely, if $r_{0}$ is a positive number such that $\left\{B_{\mathcal{S}}\left(p, r_{0}\right)\right\}_{p \in I}$ is a family of pairwise disjoint and simply connected balls in $\mathcal{S}$, then we have that

$$
h(\mathcal{R}) \leq \frac{h(\mathcal{S})}{\tanh ^{2}\left(\frac{r_{0}}{4}\right)}+\frac{2 \pi}{r_{0} \log \frac{\tanh r_{0}}{\tanh \left(\frac{r_{0}}{4}\right)}} .
$$

The difficult implication in this theorem is to prove that $\mathcal{R}$ has HII. Our proof of this consists of finding a relationship between the Poincaré metrics of $\mathcal{R}$ and $\mathcal{S}$. Far from the points in $I$ both metrics are comparable (see Lemma 3.1 below). Close to these isolated points they are not comparable but, in fact, there exists a very precise relation between the $\mathcal{S}$-balls centered at points in $I$ and its corresponding collars in $\mathcal{R}$ (see Proposition 1 in Section 6).

We start by studying the relationship between the Poincaré metrics of $\mathcal{R}$ and $\mathcal{S}$.

Lemma 3.1. Let $\mathcal{S}$ be a hyperbolic Riemann surface, let $C$ be a closed non-empty subset of $\mathcal{S}$ and $\mathcal{S}^{*}=\mathcal{S} \backslash C$. Let us consider a positive number $\varepsilon$. Then we have that

$$
\tanh \frac{\varepsilon}{2}<\frac{L_{\mathcal{S}}(\gamma)}{L_{\mathcal{S}^{*}}(\gamma)}<1
$$

for every curve $\gamma \subset \mathcal{S}$ with finite length in $\mathcal{S}$ such that $d_{\mathcal{S}}(\gamma, C) \geq \varepsilon$, and

$$
\left(\tanh \frac{\varepsilon}{2}\right)^{2}<\frac{A_{\mathcal{S}}(D)}{A_{\mathcal{S}^{*}}(D)}<1
$$

for every domain $D \subset \mathcal{S}$ with finite area in $\mathcal{S}$ such that $d_{\mathcal{S}}(D, C) \geq \varepsilon$.

ProOf. We prove Lemma 3.1 in local coordinates.

Let us fix $p \in \mathcal{S}$ with $d_{\mathcal{S}}(p, C) \geq \varepsilon$ and let us consider a local chart $\phi: V \longrightarrow \mathbb{C}$ with $\phi(p)=0$.

Let $F: \mathbb{D} \longrightarrow \mathcal{S}$ be a universal covering map with $F(0)=p$. The set $C^{\prime}=F^{-1}(C)$ is a closed subset of the unit disk. Obviously the euclidean ball $B(0, \tanh (\varepsilon / 2))=B_{\mathbb{D}}(0, \varepsilon)$ is a connected component of $F^{-1}\left(B_{\mathcal{S}}(p, \varepsilon)\right)$; it is contained in $\mathbb{D} \backslash C^{\prime}$ and the mapping $F: \mathbb{D} \backslash C^{\prime} \longrightarrow$ $\mathcal{S}^{*}$ is a covering map with $F(0)=p$. Let $G: \mathbb{D} \longrightarrow \mathbb{D} \backslash C^{\prime}$ be a 
universal covering map with $G(0)=0$. We have that $F \circ G: \mathbb{D} \longrightarrow \mathcal{S}^{*}$ is a universal covering map with $(F \circ G)(0)=p$.

Let us consider the Poincaré metrics $\lambda_{\mathcal{S}}(z)|d z|$ and $\lambda_{\mathcal{S}^{*}}(z)|d z|$ in local coordinates $(z \in \phi(V))$. Then

$$
\begin{gathered}
\lambda_{\mathcal{S}}((\phi \circ F)(0))\left|(\phi \circ F)^{\prime}(0)\right|=\lambda_{\mathbb{D}}(0), \\
\lambda_{\mathcal{S}^{*}}((\phi \circ F \circ G)(0))\left|(\phi \circ F \circ G)^{\prime}(0)\right|=\lambda_{\mathbb{D}}(0),
\end{gathered}
$$

and this gives

$$
\lambda_{\mathcal{S}}(0)\left|(\phi \circ F)^{\prime}(0)\right|=2, \quad \lambda_{\mathcal{S}^{*}}(0)\left|(\phi \circ F)^{\prime}(0)\right|\left|G^{\prime}(0)\right|=2 .
$$

These last equalities give Lemma 3.1 if we prove that $\tanh (\varepsilon / 2)<$ $\left|G^{\prime}(0)\right|<1$ since this is the infinitesimal version of (3.1) and (3.2).

Observe that $G: \mathbb{D} \longrightarrow \mathbb{D}$ satisfies $G(0)=0$. Schwarz's Lemma gives the inequality $\left|G^{\prime}(0)\right|<1$.

Recall that the simply connected set $B(0, \tanh (\varepsilon / 2))$ is contained in $\mathbb{D} \backslash C^{\prime}$. Therefore, there exists a well defined local inverse $G^{-1}$ : $B(0, \tanh (\varepsilon / 2)) \longrightarrow \mathbb{D}$ verifying $G^{-1}(0)=0$. Using again Schwarz's Lemma we obtain that

$$
\left|\left(G^{-1}\right)^{\prime}(0)\right|=\frac{1}{\left|G^{\prime}(0)\right|}<\operatorname{cotanh}\left(\frac{\varepsilon}{2}\right) .
$$

This finishes the proof of Lemma 3.1.

Proof of Theorem 1 . We begin with the proof that if $\mathcal{R}$ has HII then $\mathcal{S}$ has it and $I$ is strongly uniformly separated in $\mathcal{S}$.

We shall prove first that $I$ is a discrete set. In fact, if this is not the case, then $I$ is not strongly uniformly separated and, as we shall see, this implies that $\mathcal{R}$ has not HII, a contradiction.

Let us assume that $I$ is not a discrete set. Let $F: \mathbb{D} \longrightarrow \mathcal{S}$ be a universal covering map and let $J$ be the preimage of $I$ by $F$. Then $F: \mathbb{D} \backslash J \longrightarrow \mathcal{R}$ is a covering map. Therefore, $\delta(\mathbb{D} \backslash J) \leq \delta(\mathcal{R})$ (see, for example [FR, p. 181]). Obviously, $J$ is a closed, countable and non discrete subset of $\mathbb{D}$. Let $z_{0}$ be an accumulation point of $J$ in $\mathbb{D}$. Then, we have that $B\left(z_{0}, r\right) \cap \partial(\mathbb{D} \backslash J)=B\left(z_{0}, r\right) \cap J$ is countable, for $0<r<1-\left|z_{0}\right|$, and therefore it has zero logarithmic capacity. [FR, Theorem 4] implies that $1=\delta(\mathbb{D} \backslash J) \leq \delta(\mathcal{R}) \leq 1$. But, if $\delta(\mathcal{R})=1$, a fortiori, $\mathcal{R}$ has not HII. 
A theorem of Patterson [P2, Theorem 4.1] gives that $\delta(\mathcal{S}) \leq \delta(\mathcal{R})$, since $I$ is discrete. Therefore $\delta(\mathcal{S})<1$ and $\mathcal{S}$ has HII.

Suppose that the discrete set $I$ is not strongly uniformly separated. Let us see that, then, $\mathcal{R}$ has not HII, a contradiction. Denote again by $F: \mathbb{D} \longrightarrow \mathcal{S}$ a universal covering map and by $J$ the preimage of $I$ by $F$. As before $F: \mathbb{D} \backslash J \longrightarrow \mathcal{R}$ is a covering map and therefore, $\delta(\mathbb{D} \backslash J) \leq \delta(\mathcal{R})$ (see, for example again [FR, p. 181]). We have that for each $\varepsilon>0$, there exist points $p, q \in I$ such that either $d_{\mathcal{S}}(p, q)<\varepsilon$ or $B_{\mathcal{S}}(p, \varepsilon)$ is not simply connected. This implies that there exist $z, w \in J$ such that $d_{\mathbb{D}}(z, w)<\varepsilon$, i.e. that $J$ is not uniformly separated in $\mathbb{D}$. $[\mathrm{FR}$, Theorem 4] implies again that $\delta(\mathcal{R}) \geq \delta(\mathbb{D} \backslash J)=1$.

Let us assume now that $\mathcal{S}$ has HII and $I$ is strongly uniformly separated in $\mathcal{S}$. We want to prove that then $\mathcal{R}$ has also HII.

Let $\mathcal{D}$ be an open subset of $\mathcal{R}$ with finite area. In order to check (1.1) for $\mathcal{D}$, we can assume without loss of generality that $\mathcal{D}$ is not simply or doubly connected since this particular type of subsets always satisfy HII with constant 1 [FR, Lemma 1.1]. We can also suppose that $\partial \mathcal{D}=\gamma_{1} \cup \gamma_{2} \cup \cdots \cup \gamma_{k}$ where the simple closed curves $\gamma_{j}$ are not homotopic to the trivial loop and does not "surround" only a puncture. In fact, if this would be the case for $\gamma_{j}$, say, we could join to $\mathcal{D}$ the simply or doubly connected open set whose boundary is $\gamma_{j}$, obtaining by this way a new domain with greater area and whose boundary had less length.

Let us consider a positive number $r_{0}$ such that the balls $B_{\mathcal{S}}\left(p, r_{0}\right)$ with $p \in I$ are simply connected and pairwise disjoint. Let $\tilde{\mathcal{S}}$ be the subset of $\mathcal{R}$ given by $\tilde{\mathcal{S}}=\mathcal{S} \backslash \cup_{p \in I} B_{\mathcal{S}}\left(p, r_{0} / 2\right)$. Let $J, J_{1}, J_{2}$, be the subsets of $I$ defined by

$$
\begin{aligned}
& J=\left\{p \in I: \mathcal{D} \cap B_{\mathcal{S}}\left(p, r_{0} / 2\right)^{*} \neq \varnothing\right\}, \\
& J_{1}=\left\{p \in J: B_{\mathcal{S}}\left(p, r_{0} / 2\right)^{*} \subset \mathcal{D}\right\}, \\
& J_{2}=\left\{p \in J: \partial \mathcal{D} \cap B_{\mathcal{S}}\left(p, r_{0} / 2\right)^{*} \neq \varnothing\right\} .
\end{aligned}
$$

It is obvious that $\left\{J_{1}, J_{2}\right\}$ is a partition of $J$.

First of all we remark that

$$
L_{\mathcal{S}}\left(\partial \mathcal{D} \cap B_{\mathcal{S}}\left(p, r_{0}\right)\right) \geq r_{0}, \quad \text { for all } p \in J_{2} .
$$

To see this, suppose that $L_{\mathcal{S}}\left(\partial \mathcal{D} \cap B_{\mathcal{S}}\left(p, r_{0}\right)\right)<r_{0}$ for some $p \in J_{2}$. Then, we have that there exists a boundary curve $\gamma_{j}$ with $L_{\mathcal{S}}\left(\gamma_{j}\right)<r_{0}$; such a curve must verify that $\gamma_{j} \subset B_{\mathcal{S}}\left(p, r_{0}\right)$ since

$$
d_{\mathcal{S}}\left(\partial B_{\mathcal{S}}\left(p, r_{0}\right), \partial B_{\mathcal{S}}\left(p, \frac{r_{0}}{2}\right)\right)=\frac{r_{0}}{2} .
$$


But, if $\gamma_{j} \subset B_{\mathcal{S}}\left(p, r_{0}\right)$, then $\gamma_{j}$ is homotopic in $\mathcal{R}$ to zero or to $p$, and this is not possible.

Claim. There exists a constant $c$, which only depends on $r_{0}$ and neither on $\mathcal{S}$ nor $I$, such that

$$
A_{\mathcal{R}}\left(B_{\mathcal{S}}\left(p, \frac{r_{0}}{2}\right)^{*}\right) \leq c, \quad \text { for every } p \in I .
$$

Then we have that

$$
A_{\mathcal{R}}\left(B_{\mathcal{S}}\left(p, \frac{r_{0}}{2}\right)^{*}\right) \leq \frac{c}{4 \pi \sinh ^{2}\left(\frac{r_{0}}{4}\right)} A_{\mathcal{S}}\left(B_{\mathcal{S}}\left(p, \frac{r_{0}}{2}\right)\right),
$$

for every $p \in J_{1}$; since $B_{\mathcal{S}}\left(p, r_{0} / 2\right)$ is simply connected and then

$$
A_{\mathcal{S}}\left(B_{\mathcal{S}}\left(p, \frac{r_{0}}{2}\right)\right)=A_{\mathbb{D}}\left(B_{\mathbb{D}}\left(0, \frac{r_{0}}{2}\right)\right)=4 \pi \sinh ^{2}\left(\frac{r_{0}}{4}\right),
$$

and by $(3.3)$

$$
A_{\mathcal{R}}\left(B_{\mathcal{S}}\left(p, \frac{r_{0}}{2}\right)^{*}\right) \leq \frac{c}{r_{0}} L_{\mathcal{S}}\left(\partial \mathcal{D} \cap B_{S}\left(p, r_{0}\right)\right),
$$

for every $p \in J_{2}$. Using (3.2), (3.5) and (3.6), we have that

$$
\begin{aligned}
A_{\mathcal{R}}(\mathcal{D}) \leq & A_{\mathcal{R}}(\mathcal{D} \cap \tilde{\mathcal{S}})+\sum_{p \in J_{1}} A_{\mathcal{R}}\left(B_{\mathcal{S}}\left(p, \frac{r_{0}}{2}\right)^{*}\right)+\sum_{p \in J_{2}} A_{\mathcal{R}}\left(B_{\mathcal{S}}\left(p, \frac{r_{0}}{2}\right)^{*}\right) \\
\leq & \frac{1}{\tanh ^{2}\left(\frac{r_{0}}{4}\right)} A_{\mathcal{S}}(\mathcal{D} \cap \tilde{\mathcal{S}}) \\
& +\frac{c}{4 \pi \sinh ^{2}\left(\frac{r_{0}}{4}\right)} \sum_{p \in J_{1}} A_{\mathcal{S}}\left(B_{\mathcal{S}}\left(p, \frac{r_{0}}{2}\right)\right)+\frac{c}{r_{0}} L_{\mathcal{S}}(\partial \mathcal{D}) .
\end{aligned}
$$

Let $H$ be

$$
H=\max \left\{\frac{1}{\tanh ^{2}\left(\frac{r_{0}}{4}\right)}, \frac{c}{4 \pi \sinh ^{2}\left(\frac{r_{0}}{4}\right)}\right\} .
$$

Therefore

$$
\begin{aligned}
A_{\mathcal{R}}(\mathcal{D}) & \leq H\left(A_{\mathcal{S}}(\mathcal{D} \cap \tilde{\mathcal{S}})+\sum_{p \in J_{1}} A_{\mathcal{S}}\left(B \mathcal{S}\left(p, \frac{r_{0}}{2}\right)\right)\right)+\frac{c}{r_{0}} L_{\mathcal{S}}(\partial \mathcal{D}) \\
& \leq H A_{\mathcal{S}}(\mathcal{D})+\frac{c}{r_{0}} L_{\mathcal{S}}(\partial \mathcal{D}) \\
& \leq H h(\mathcal{S}) L_{\mathcal{S}}(\partial \mathcal{D})+\frac{c}{r_{0}} L_{\mathcal{S}}(\partial \mathcal{D}) \\
& \leq\left(H h(\mathcal{S})+\frac{c}{r_{0}}\right) L_{\mathcal{R}}(\partial \mathcal{D})
\end{aligned}
$$


Then we have that $\mathcal{R}$ has HII with constant

$$
h(\mathcal{R}) \leq H h(\mathcal{S})+\frac{c}{r_{0}} .
$$

To finish the proof of Theorem 1 we only need to prove (3.4) with

$$
c=\frac{2 \pi}{\log \frac{\tanh r_{0}}{\tanh \left(\frac{r_{0}}{4}\right)}},
$$

since we will see below that, with this value of $c$, we have that

$$
H=\frac{1}{\tanh ^{2}\left(\frac{r_{0}}{4}\right)} .
$$

Lemma 3.2. Let $\mathcal{S}$ be a hyperbolic Riemann surface and $\left\{B_{\mathcal{S}}\left(p, r_{0}\right)\right\}_{p \in I}$ be a disjoint family of simply connected balls in $\mathcal{S}$. If $\mathcal{R}=\mathcal{S} \backslash I$, then we have that

$$
A_{\mathcal{R}}\left(B_{\mathcal{S}}(p, r)^{*}\right) \leq \frac{2 \pi}{\log \frac{\tanh r_{0}}{\tanh \left(\frac{r}{2}\right)}}, \quad \text { for } 0<r \leq r_{0}
$$

Proof. Let us fix a point $p \in I$. Let us consider a universal covering map $F: \mathbb{D} \longrightarrow \mathcal{S}$ such that $F(0)=p$. Let $J$ be the preimage of $I$ by $F$. The intersection of the ball $F^{-1}\left(B_{\mathcal{S}}\left(p, 2 r_{0}\right)\right)=B_{\mathbb{D}}\left(0,2 r_{0}\right)=$ $B\left(0, \tanh r_{0}\right)$ with the set $J$ is exactly $\{0\}$. Since $F: \mathbb{D} \backslash J \longrightarrow \mathcal{R}$ is a covering map, it follows that for $0<r \leq r_{0}$

$$
\begin{aligned}
A_{\mathcal{R}}\left(B_{\mathcal{S}}(p, r)^{*}\right) & =A_{\mathbb{D} \backslash J}\left(B_{\mathbb{D}}(0, r)^{*}\right) \\
& \leq A_{B\left(0, \tanh r_{0}\right)^{*}}\left(B_{\mathbb{D}}(0, r)^{*}\right) \\
& =A_{B\left(0, \tanh r_{0}\right)^{*}}\left(B\left(0, \tanh \left(\frac{r}{2}\right)\right)^{*}\right) \\
& =A_{\mathbb{D}{ }^{*}}\left(B\left(0, \frac{\tanh \left(\frac{r}{2}\right)}{\tanh r_{0}}\right)^{*}\right) \\
& =\int_{B\left(0, \tanh (r / 2) / \tanh r_{0}\right)^{*}} \frac{d x d y}{(|z| \log |z|)^{2}} \\
& =\frac{2 \pi}{\log \frac{\tanh r_{0}}{\tanh \left(\frac{r}{2}\right)}} .
\end{aligned}
$$


This finishes the proof of Lemma 3.2.

The estimate (3.4) follows now from Lemma 3.2 with

$$
c=\frac{2 \pi}{\log \frac{\tanh r_{0}}{\tanh \left(\frac{r_{0}}{4}\right)}},
$$

and therefore (3.7) and (3.8) give the inequality in Theorem 1, if

$$
H=\frac{1}{\tanh ^{2}\left(\frac{r_{0}}{4}\right)} .
$$

In order to prove this, we only need to check that

$$
\frac{1}{\tanh ^{2}\left(\frac{r_{0}}{4}\right)} \geq \frac{1}{2 \sinh ^{2}\left(\frac{r_{0}}{4}\right) \log \frac{\tanh r_{0}}{\tanh \left(\frac{r_{0}}{4}\right)}}
$$

and this follows from the fact that

$$
G(x)=2 \log \frac{\tanh x}{\tanh \left(\frac{x}{4}\right)}-\frac{1}{\cosh ^{2}\left(\frac{x}{4}\right)} \geq 0, \quad \text { for } x>0 .
$$

This inequality is a consequence of the fact that

$$
\begin{aligned}
G^{\prime}(x) & =\frac{4}{2 \sinh x \cosh x}-\frac{1}{2 \sinh \left(\frac{x}{4}\right) \cosh ^{3}\left(\frac{x}{4}\right)} \\
& =\frac{4}{\sinh (2 x)}-\frac{4}{2 \sinh \left(\frac{x}{2}\right)+\sinh x} \\
& \leq 0
\end{aligned}
$$

and

$$
\lim _{x \rightarrow \infty} G(x)=0
$$

REMARK. The inequality (3.4) can be obtained alternatively from Proposition 1. This proposition will be stated and proved in Section 
6. We have used here Lemma 3.2 since it gives best estimates in this context.

\section{Proof of Theorem 3.}

Theorem 3. Let $\Omega$ be an admissible Denjoy domain, let I be the set of isolated points of $\partial \Omega$ and $\Omega_{0}=\Omega \cup I$.

a) If $\Omega$ has HII, then there exists a positive constant $c$ such that for any border set of $\partial \Omega_{0}, B=\left\{b_{1}, \ldots, b_{2 n}\right\}$ with $n \geq 3$, we have that

$$
\frac{1}{n} \sum_{j=1}^{n} \Phi_{1}\left(r\left(\left\{b_{2 j-1}, b_{2 j}, b_{2 j+1}, b_{2 j+2}\right\}\right)\right)>c .
$$

b) If there exists a positive constant $c$ such that for any border set of $\partial \Omega_{0}, B=\left\{b_{1}, \ldots, b_{2 n}\right\}$ with $n \geq 3$, we have that

$$
\frac{1}{n} \sum_{j=1}^{n} \Phi_{2}\left(r\left(\left\{b_{2 j-1}, b_{2 j}, b_{2 j+1}, b_{2 j+2}\right\}\right)\right)>c,
$$

then $\Omega$ has HII.

Theorem 3 is a direct consequence of Theorem 1 and the following result.

Theorem 2. Let $\Omega$ be a Denjoy domain such that $\partial \Omega$ has no isolated points. Then

1) If $\Omega$ has HII, then there exists a positive constant $c$ such that for any border set of $\partial \Omega, B=\left\{b_{1}, \ldots, b_{2 n}\right\}(n \geq 3)$, we have that

$$
\frac{1}{n} \sum_{j=1}^{n} \Phi_{1}\left(r\left(\left\{b_{2 j-1}, b_{2 j}, b_{2 j+1}, b_{2 j+2}\right\}\right)\right)>c .
$$

2) If there exists a positive constant $c$ such that for any border set of $\partial \Omega, B=\left\{b_{1}, \ldots, b_{2 n}\right\}(n \geq 3)$, we have that

$$
\frac{1}{n} \sum_{j=1}^{n} \Phi_{2}\left(r\left(\left\{b_{2 j-1}, b_{2 j}, b_{2 j+1}, b_{2 j+2}\right\}\right)\right)>c,
$$


then $\Omega$ has HII.

The proof of Theorem 2 has three main ideas. The first one (see Lemma 4.1) is to reduce dramatically the set of domains in which we must check (1.1). Secondly, we will establish a bijective correspondence between these domains and border sets (see Lemma 4.2). Finally, we relate the length of each boundary curve of these domains with the length of some curves in some extremal domains which is given by the functions $\Phi_{1}$ and $\Phi_{2}$ (see Lemmas 4.3 and 4.5).

A geodesic domain in a Riemann surface $\mathcal{S}$ is a domain $G \subset \mathcal{S}$ (which is not simply or doubly connected) such that $\partial G$ consists of finitely many simple closed geodesics, and $A_{\mathcal{S}}(G)$ is finite. $G$ does not have to be relatively compact since it may "surround" finitely many punctures (isolated points in $\partial \mathcal{S}$ in the case that $\mathcal{S} \subset \hat{\mathbb{C}}$ ). We can think of a puncture as a boundary geodesic of zero length. Recall that if $\gamma$ is a closed curve in $\mathcal{S}$ and $[\gamma]$ denotes its free homotopy class in $\mathcal{S}$, then there is a unique simple closed geodesic of minimal length in the class, unless $\gamma$ is homotopic to zero or surrounds only a puncture; in these cases it is not possible to find such geodesic because there are curves in the class with arbitrary small length.

In [FR, Lemma 1.2] it was proved that if $\mathcal{S}$ verifies (1.1) for geodesic domains, then it verifies HII. In fact, if $h_{g}(\mathcal{S})$ is the infimum of the constants $h$ such that the inequality

$$
A_{\mathcal{S}}(G) \leq h L_{\mathcal{S}}(\partial G)
$$

is true for any geodesic domain $G$, we have that

$$
h(\mathcal{S}) \leq h_{g}(\mathcal{S})+2
$$

We shall prove now that if a Denjoy domain $\Omega$ verifies (1.1) for geodesic domains which are symmetric with respect to the real axis (SGdomains), then $\Omega$ verifies (1.1) for any geodesic domain and therefore it verifies HII.

In fact, we have the following result, which is true even if $\partial \Omega$ has isolated points.

Lemma 4.1. Let $\Omega$ be a Denjoy domain satisfying the inequality

$$
A_{\Omega}(G) \leq h L_{\Omega}(\partial G)
$$

for every SG-domain $G$ in $\Omega$ and for a positive constant $h$. 
Then $\Omega$ satisfies HII with

$$
h_{g}(\Omega) \leq 2 h \quad \text { and } \quad h(\Omega) \leq 2 h+2 .
$$

Proof. Let $G$ be a geodesic domain in $\Omega$. Without loss of generality we can suppose that $G$ contains the point at infinity. Let us consider the family $\mathcal{F}_{1}$ of subarcs of $\partial G$ which joins two points of the real axis and are contained either in $\{z: \operatorname{Im} z \geq 0\}$ or in $\{z: \operatorname{Im} z \leq 0\}$ and reflect each of them with respect to the real axis. We obtain in this way a family of closed curves $\mathcal{F}_{2}$. Let $\mathcal{F}_{3}$ be the family constituted by all simple closed geodesics in $\Omega$ which are freely homotopic to some curve of $\mathcal{F}_{2}$. We construct now a new family $\mathcal{F}_{4}$ from $\mathcal{F}_{3}$ in the following way: a curve $\gamma$ of $\mathcal{F}_{3}$ belongs to $\mathcal{F}_{4}$ if and only if the bounded (in the euclidean sense) Jordan domain $\mathcal{J}$ such that $\partial \mathcal{J}=\gamma$ does not contain any other curve in $\mathcal{F}_{3}$ and $\mathcal{J} \cap \partial \Omega$ is not a finite set. Observe that the negative curvature implies that any two geodesics $\gamma_{1}, \gamma_{2}$ in $\mathcal{F}_{3}$ are disjoint; therefore either $\mathcal{J}_{1}$ and $\mathcal{J}_{2}$ are disjoint, either $\mathcal{J}_{1} \subset \mathcal{J}_{2}$ or $\mathcal{J}_{2} \subset \mathcal{J}_{1}$. Let $G_{0}$ be the SG-domain whose boundary is constituted by the curves in $\mathcal{F}_{4}$.

To illustrate this construction, let us consider for example the geodesic domain $G$ shown as the exterior of the curves in this picture.
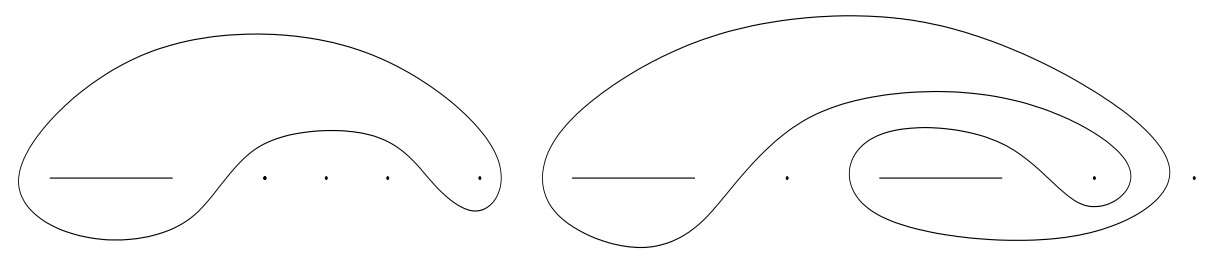

Then, the family of curves $\mathcal{F}_{2}$ looks like the following
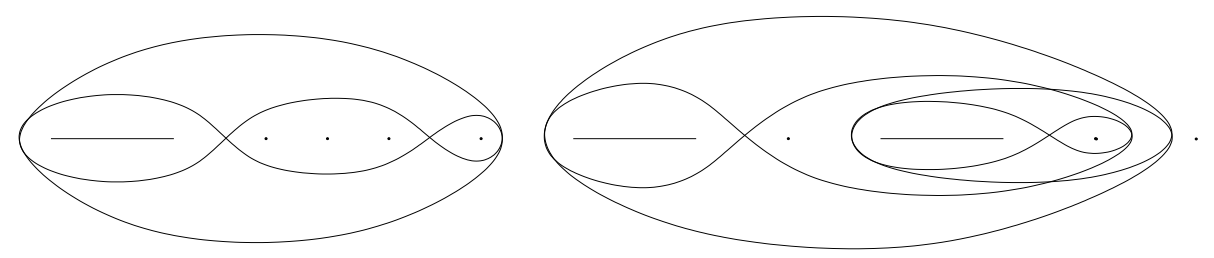

The family of simple closed geodesics $\mathcal{F}_{3}$ is shown by 

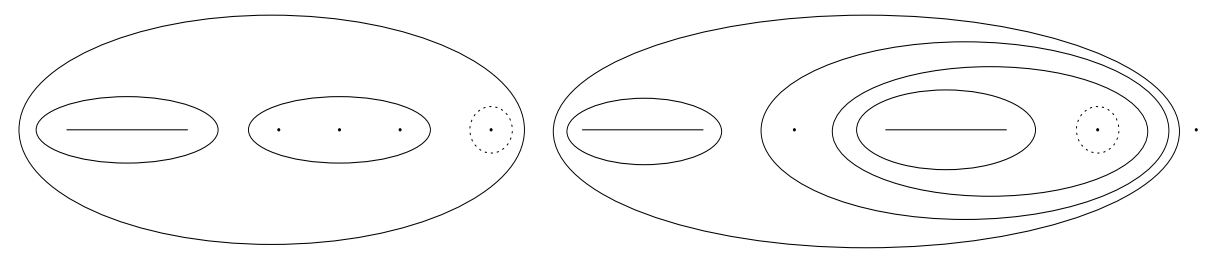

Note that the dotted curves in the last picture represent the free homotopy classes without geodesics; they are not in $\mathcal{F}_{3}$. Finally, the geodesic domain $G_{0}$ is the exterior of the geodesics in
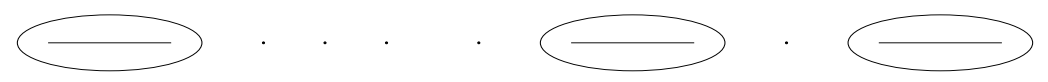

It is clear that

$$
L_{\Omega}\left(\partial G_{0}\right) \leq 2 L_{\Omega}(\partial G)
$$

Let now $n, p$ be, respectively, the number of simple closed geodesics limiting $G$ and the number of punctures in $G$. Let also $n_{0}, p_{0}$ be the corresponding numbers for $G_{0}$. Observe that $n_{0}+p_{0} \geq n+p$. To see this, let us consider the set $\Gamma(G)$ of generalized geodesics limiting $G$, i.e. the union of the set of $n$ geodesics in $\partial G$ and the set of $p$ punctures "surrounded" by $G$. We want to show that

$$
\operatorname{card} \Gamma(G) \leq \operatorname{card} \Gamma\left(G_{0}\right)
$$

If a puncture is surrounded by $G$ it is also surrounded by $G_{0}$. On the other hand, given a geodesic $\gamma$ of $\partial G$ let us consider the bounded (in the euclidean sense) Jordan domain $\mathcal{J}$ with $\partial \mathcal{J}=\gamma$; if the intersection of $\mathcal{J}$ with the real axis has $m$ connected components, the geodesic $\gamma$ "generates" at least $m$ generalized geodesics of $\partial G_{0}$. This gives that $n_{0}+p_{0} \geq n+p$.

Gauss-Bonnet theorem gives that

$$
A_{\Omega}\left(G_{0}\right)=2 \pi\left(n_{0}+p_{0}-2\right) \geq 2 \pi(n+p-2)=A_{\Omega}(G),
$$

since the hyperbolic metric of $\Omega$ has curvature -1 .

Therefore

$$
A_{\Omega}(G) \leq A_{\Omega}\left(G_{0}\right) \leq h L_{\Omega}\left(\partial G_{0}\right) \leq 2 h L_{\Omega}(\partial G)
$$

and so we have proved the first inequality in Lemma 4.1. The second inequality is a consequence of the first one and [FR, Lemma 1.2]. 
Given a border set of $\partial \Omega$ with four points, $B=\left\{b_{1}, b_{2}, b_{3}, b_{4}\right\}$, we denote by $\gamma(B)$ the unique simple closed geodesic in $\Omega$ which separates $\left[b_{2}, b_{3}\right]$ from $\left[b_{4}, b_{1}\right]$.

Lemma 4.2. A Denjoy domain $\Omega$ such that $\partial \Omega$ has no isolated points has HII if and only if there exists a positive constant $c$ such that for any border set of $\partial \Omega, B=\left\{b_{1}, \ldots, b_{2 n}\right\}$ with $n \geq 3$, we have that

$$
\frac{1}{n} \sum_{j=1}^{n} L_{\Omega}\left(\gamma\left(\left\{b_{2 j-1}, b_{2 j}, b_{2 j+1}, b_{2 j+2}\right\}\right)\right)>c .
$$

Proof. Observe that we can establish a one to one correspondence between border sets of $\partial \Omega$ with $n \geq 3$ and SG-domains in $\Omega$. Given a border set $B$ of $\partial \Omega, B=\left\{b_{1}, \ldots, b_{2 n}\right\}$, let us consider the set of $n$ geodesics $(n \geq 3)$

$$
\mathcal{G}=\left\{\gamma\left(\left\{b_{2 j-1}, b_{2 j}, b_{2 j+1}, b_{2 j+2}\right\}\right): j=1, \ldots, n\right\} .
$$

The curves in $\mathcal{G}$ limit a geodesic domain $G$ associated to $B$. Observe that if $n=2$, both geodesics are the same and then obviously they do not limit a geodesic domain.

It is clear that this process has a well defined inverse. GaussBonnet theorem gives that $A(G)=2 \pi(n-2)$. Therefore, we have that $A(G) \asymp n$. This fact and Lemma 4.1 give Lemma 4.2.

It is clear that if we define

$$
\begin{aligned}
& \Omega_{1}=\hat{\mathbb{C}} \backslash\left(\left[b_{2 j}, b_{2 j+1}\right] \cup\left[b_{2 j+2}, b_{2 j-1}\right]\right), \\
& \Omega_{2}=\hat{\mathbb{C}} \backslash\left\{b_{2 j-1}, b_{2 j}, b_{2 j+1}, b_{2 j+2}\right\},
\end{aligned}
$$

then we have

$$
\begin{aligned}
L_{\Omega_{2}}\left(\gamma\left(\left\{b_{2 j-1}, b_{2 j}, b_{2 j+1}, b_{2 j+2}\right\}\right)\right) & \leq L_{\Omega}\left(\gamma\left(\left\{b_{2 j-1}, b_{2 j}, b_{2 j+1}, b_{2 j+2}\right\}\right)\right) \\
& \leq L_{\Omega_{1}}\left(\gamma\left(\left\{b_{2 j-1}, b_{2 j}, b_{2 j+1}, b_{2 j+2}\right\}\right)\right)
\end{aligned}
$$

since $\Omega_{1} \subset \Omega \subset \Omega_{2}$.

In order to prove Theorem 2 we only need to relate the length in $\Omega_{1}$ and $\Omega_{2}$ of the geodesic

$$
\gamma\left(\left\{b_{2 j-1}, b_{2 j}, b_{2 j+1}, b_{2 j+2}\right\}\right)
$$


with the functions $\Phi_{1}$ and $\Phi_{2}$ (see their definitions after (2.1)).

The following result gives an estimate of the hyperbolic length of the imaginary axis in some normalized Denjoy domains. This curve is important because it is the geodesic (in many symmetric cases (see, e.g. Lemma 4.5)) whose length we want to estimate.

We recall that $[x]$ denotes the greatest natural number which is less or equal than $x$.

Lemma 4.3. Let us fix a number $0<a<1$ and let $0<t<1$. For each natural number $m$ such that

$$
m \leq N=\left[\frac{\log \frac{a}{t}}{\log \frac{1}{a}}\right]
$$

let us consider the closed set

$D_{m}=D_{m}(t)=\left\{z \in \mathbb{C}: a^{m+1} \leq|z+t| \leq a^{m}\right.$ or $\left.a^{m+1} \leq|z-t| \leq a^{m}\right\}$.

Let $\Omega$ be a Denjoy domain such that $\{-1,-t, t, 1\} \subset \partial \Omega \subset[-1,-t] \cup$ $[t, 1]$.

Let $n_{1}<n_{2}<\cdots<n_{\ell-1}$ be all the natural numbers in $(0, N)$ satisfying $D_{n_{j}} \cap \partial \Omega \neq \varnothing, n_{0}=0$ and $n_{\ell}=N$.

Then we have that there exists a universal constant $0<t_{0}<1$ such that if we denote by $\sigma$ the imaginary axis with the point at infinity included, then

$$
L_{\Omega}(\sigma) \asymp \sum_{j=1}^{\ell}\left(1+\log \left(n_{j}-n_{j-1}\right)\right), \quad \text { for } 0<t \leq t_{0}
$$

Here the constant in $\asymp$ depends only on a but neither on $\Omega$ nor $t$.

Proof. The idea of the proof is to estimate the length of "dyadic" segments of the curve. Over each one of these segments we shall have a precise estimate of the distance to the boundary of the domain and the function $\beta_{\Omega}$ (see (2.2)). These facts and [BP, Theorem 1] will give the lemma up to a technical detail involving the point at infinity which we solve in Lemma 4.4. 
Let $I_{m}=\sigma \cap D_{m}, 0 \leq m \leq N$. We are going to estimate $L_{\Omega}\left(I_{m}\right)$, the length in $\Omega$ of $I_{m}$, under the assumptions that

$$
\begin{gathered}
\left(D_{m-k} \cup D_{m+k}\right) \cap \partial \Omega \neq \varnothing, \\
\left(D_{m-k+1} \cup D_{m-k+2} \cup \cdots \cup D_{m-1}\right. \\
\left.\cup D_{m} \cup D_{m+1} \cup \cdots \cup D_{m+k-1}\right) \cap \partial \Omega=\varnothing,
\end{gathered}
$$

for $0 \leq k \leq \min \{m, N-m\}$ (obviously the second condition does not appear if $k=0$ ).

Let $\Omega^{*}:=\Omega \backslash\{\infty\}$; the computations in $\Omega^{*}$ are easier than in $\Omega$ because we can apply $[\mathrm{BP}$, Theorem 1$]$ since $\Omega^{*} \subset \mathbb{C}$.

Let us consider a point $b \in\left(D_{m-k} \cup D_{m+k}\right) \cap \partial \Omega$. We have four possibilities:

i) $b \in D_{m+k}$ and $a^{m+k+1} \leq|b+t| \leq a^{m+k}$,

ii) $b \in D_{m+k}$ and $a^{m+k+1} \leq|b-t| \leq a^{m+k}$,

iii) $b \in D_{m-k}$ and $a^{m-k+1} \leq|b+t| \leq a^{m-k}$,

iv) $b \in D_{m-k}$ and $a^{m-k+1} \leq|b-t| \leq a^{m-k}$.

We consider now the case i). If $z \in I_{m}$, it satisfies inequalities $a^{m+1} \leq|z+t| \leq a^{m}$ (in fact, $z$ satisfies both inequalities in the definition of $\left.D_{m}\right)$, and then

$$
\frac{1}{a^{k-1}}=\frac{a^{m+1}}{a^{m+k}} \leq \frac{|z+t|}{|b+t|} \leq \frac{a^{m}}{a^{m+k+1}}=\frac{1}{a^{k+1}} .
$$

This implies that

$$
1+\beta_{\Omega^{*}}(z) \asymp(k+1) \log \frac{1}{a} .
$$

The same result can be deduced, with similar arguments, in the cases ii), iii) and iv).

Using (4.2) and [BP, Theorem 1] we obtain that

$$
\lambda_{\Omega^{*}}(z) \asymp \frac{1}{a^{m}(k+1)}, \quad \text { for } k \geq 0 .
$$

Next we are going to estimate the euclidean length of $I_{m}$

$$
\begin{aligned}
\left|I_{m}\right| & =\sqrt{a^{2 m}-t^{2}}-\sqrt{a^{2 m+2}-t^{2}} \\
& =\frac{a^{2 m}\left(1-a^{2}\right)}{\sqrt{a^{2 m}-t^{2}}+\sqrt{a^{2 m+2}-t^{2}}} .
\end{aligned}
$$


Observe that (4.1) gives $t^{2} \leq a^{2 m+2}$. This fact and (4.4) imply that $\left|I_{m}\right| \asymp a^{m}$, and therefore

$$
L_{\Omega^{*}}\left(I_{m}\right)=\int_{I_{m}} \lambda_{\Omega^{*}}(z)|d z| \asymp \int_{I_{m}} \frac{|d z|}{a^{m}(k+1)} \asymp \frac{1}{k+1} .
$$

In order to estimate $L_{\Omega}\left(I_{m}\right)$ we only need to prove that $\lambda_{\Omega^{*}}(z) \asymp \lambda_{\Omega}(z)$ for $|z| \leq 1$.

This last relation would be easy to prove (see Lemma 3.1 with $C=$ $\{\infty\}$ ) if we were not interested in obtaining constants independent of $\Omega$ and $t$. But, to obtain universal constants, we need a more sophisticated argument.

Lemma 4.4. Let $E$ be a closed subset of the closed unit disk such that $\{-1,-t, t, 1\} \subset E$. Then, for each $\rho>1$ there exist constants $t_{0} \in(0,1)$ and $c>0$ which only depend on $\rho$ such that

$$
\lambda_{\widehat{\mathbb{C}} \backslash E}(z) \geq c \lambda_{\mathbb{C} \backslash E}(z),
$$

for every $0<t \leq t_{0}$ and $|z| \leq \rho$.

Proof. By [He, Theorem 1] we have that

$$
\lambda_{\widehat{\mathbb{C}} \backslash\{-1,-t, t, 1\}}(z) \longrightarrow \lambda_{\widehat{\mathbb{C}} \backslash\{-1,0,1\}}(z), \quad \text { as } t \longrightarrow 0,
$$

uniformly over compact subsets of $\hat{\mathbb{C}} \backslash\{-1,0,1\}$. Therefore, for each $\rho>1$, there exist constants $t_{0}, c_{1}$ which only depend on $\rho$, such that if $0<t \leq t_{0}$ and $\gamma$ is a curve contained in $\{w \in \hat{\mathbb{C}}:|w| \geq \rho\}$, then

$$
L_{\widehat{\mathbb{C}} \backslash\{-1,-t, t, 1\}}(\gamma) \geq c_{1} L_{\widehat{\mathbb{C}} \backslash\{-1,0,1\}}(\gamma) .
$$

On the other hand, by $[\mathrm{Br}$, Theorem 1], the set $\{w \in \hat{\mathbb{C}}:|w| \geq \rho\}$ is hyperbolically convex in every hyperbolic plane domain containing it. Hence,

$$
d_{\hat{\mathbb{C}} \backslash E}(w, \infty) \geq d_{\hat{\mathbb{C}} \backslash\{-1,-t, t, 1\}}(w, \infty) \geq c_{1} d_{\hat{\mathbb{C}} \backslash\{-1,0,1\}}(w, \infty),
$$

if $|w| \geq \rho$. Now, it is clear that there exists a positive constant $r$ which only depends on $\rho$ such that

$$
B_{\hat{\mathbb{C}} \backslash\{-1,0,1\}}\left(\infty, \frac{r}{c_{1}}\right) \subset\{w \in \hat{\mathbb{C}}:|w|>\rho\} .
$$


This fact and (4.6) says that

$$
B_{\widehat{\mathbb{C}} \backslash E}(\infty, r) \subset\{w \in \hat{\mathbb{C}}:|w|>\rho\},
$$

and so, if $|z| \leq \rho$, we have that $d_{\widehat{\mathbb{C}} \backslash E}(\infty, z) \geq r$. Therefore Lemma 3.1 (with $C=\{\infty\}$ ) gives that

$$
c=\tanh \left(\frac{r}{2}\right)<\frac{\lambda_{\hat{\mathbb{C}} \backslash E}(z)}{\lambda_{\mathbb{C} \backslash E}(z)}<1 .
$$

This finishes the proof of Lemma 4.4.

In what follows we will take the fixed value $\rho=2$ and we will consider the corresponding $c$ and $t_{0}$. This $t_{0}$ is the constant that works in Lemma 4.3.

Now Lemma 4.4 and (4.5) give

$$
L_{\Omega}\left(I_{m}\right) \asymp \frac{1}{k+1}, \quad \text { if } 0<t \leq t_{0} .
$$

Therefore

$L_{\Omega}\left(I_{n_{j-1}} \cup \cdots \cup I_{n_{j}}\right) \asymp 2\left(1+\frac{1}{2}+\frac{1}{3}+\cdots+\frac{2}{n_{j}-n_{j-1}}\right) \asymp 1+\log \left(n_{j}-n_{j-1}\right)$

and

$$
L_{\Omega}\left(I_{0} \cup \cdots \cup I_{N}\right) \asymp \sum_{j=1}^{\ell}\left(1+\log \left(n_{j}-n_{j-1}\right)\right) .
$$

In order to finish the proof of Lemma 4.3 it is enough to check that

$$
L_{\Omega}(\sigma) \asymp L_{\Omega}\left(I_{0} \cup \cdots \cup I_{N}\right),
$$

where the constant in $\asymp$ depends only on $a$. 
This is a consequence of the following facts.

$$
\begin{aligned}
& L_{\Omega}\left(\sigma \cap\left\{w \in \hat{\mathbb{C}}:|w| \leq \sqrt{a^{2 N+2}-t^{2}}\right\}\right) \\
& \leq L_{\hat{\mathbb{C}} \backslash[t,-t]}\left(\sigma \cap\left\{w \in \hat{\mathbb{C}}:|w| \leq a^{N+1}\right\}\right) \\
& \leq L_{\hat{\mathbb{C}} \backslash[t,-t]}\left(\sigma \cap\left\{w \in \hat{\mathbb{C}}:|w| \leq \frac{t}{a}\right\}\right) \\
& =L_{\widehat{\mathbb{C}} \backslash[1,-1]}\left(\sigma \cap\left\{w \in \hat{\mathbb{C}}:|w| \leq \frac{1}{a}\right\}\right), \\
& L_{\Omega}\left(\sigma \cap\left\{w \in \hat{\mathbb{C}}:|w| \geq \sqrt{1-t^{2}}\right\}\right) \\
& \leq L_{\hat{\mathbb{C}} \backslash[-1,1]}\left(\sigma \cap\left\{w \in \hat{\mathbb{C}}:|w| \geq \sqrt{1-t_{0}^{2}}\right\}\right), \\
& \sum_{j=1}^{\ell}\left(1+\log \left(n_{j}-n_{j-1}\right)\right) \geq 1+\log N \asymp \log \log \left(\frac{1}{t}\right), \\
& \sigma \subset I_{0} \cup \cdots \cup I_{N} \cup\left\{w \in \hat{\mathbb{C}}:|w| \leq \sqrt{a^{2 N+2}-t^{2}}\right\} \\
& \cup\left\{w \in \hat{\mathbb{C}}:|w| \geq \sqrt{1-t^{2}}\right\} .
\end{aligned}
$$

This finishes the proof of Lemma 4.3.

For a border set $B=\left\{b_{1}, b_{2}, b_{3}, b_{4}\right\}$, in order to obtain a more symmetric situation, as in Lemma 4.3 , we consider the Möbius transformations

$$
\begin{aligned}
& T(z)=T_{B}(z)=\frac{\left(b_{2}-b_{1}\right)\left(z-b_{3}\right)}{\left(b_{3}-b_{2}\right)\left(z-b_{1}\right)} \\
& T_{B}^{-1}(z)=\frac{b_{1}\left(b_{3}-b_{2}\right) z-b_{3}\left(b_{2}-b_{1}\right)}{\left(b_{3}-b_{2}\right) z-\left(b_{2}-b_{1}\right)} \\
& S(z)=S_{B}(z)=\frac{z+1-\sqrt{1+r(B)}}{z+1+\sqrt{1+r(B)}} \\
& S_{B}^{-1}(z)=\frac{-2}{1-t(B)} \frac{z+t(B)}{z-1} \\
& \quad=\frac{(-1-\sqrt{1+r(B)}) z+1-\sqrt{1+r(B)}}{z-1}, \\
& U(z)=U_{B}(z)=\left(T^{-1} \circ S^{-1}\right)(z)
\end{aligned}
$$


where $r(B)$ is defined by $(2.1)$ (observe that $\left.r(B)=T_{B}\left(b_{4}\right)\right)$ and $t(B)$ is defined by

$$
t(B)=\frac{\sqrt{1+r(B)}-1}{\sqrt{1+r(B)}+1}, \quad r(B)=\frac{4 t(B)}{(1-t(B))^{2}} .
$$

Observe that the images by $T$ of $b_{1}, b_{2}, b_{3}, b_{4}$ are $\infty,-1,0, r$ in this order, the images by $S$ of $\infty,-1,0, r$ are $1,-1,-t, t$ also in this order and therefore the images by $U$ of $1,-1,-t, t$ are $b_{1}, b_{2}, b_{3}, b_{4}$.

Lemma 4.5. For $r>0$ let $T_{r}$ be the Teichmüller annulus, i.e. $T_{r}=\mathbb{C} \backslash$ $([-1,0] \cup[r, \infty))$ and $S_{r}=\mathbb{C} \backslash\{-1,0, r\}$. Then we have that the simple closed geodesic $\sigma_{r}$ which surrounds $\{-1,0\}$ and does not surround $\{r\}$ is equal to $\{z \in \mathbb{C}:|z+1|=\sqrt{1+r}\}$ in both domains. Moreover,

$$
L_{T_{r}}\left(\sigma_{r}\right) \asymp \Phi_{1}(r), \quad L_{S_{r}}\left(\sigma_{r}\right) \asymp \Phi_{2}(r), \quad r>0 .
$$

Proof. Let us consider the images of the domains $T_{r}$ and $S_{r}$ under the Möbius transformation $S(z)$ (see (4.7)) which maps the points $-1,0, r, \infty$ to $-1,-t, t, 1$ in this order (if $r$ and $t$ are related by (4.8)). It is clear, by symmetry, that the simple closed geodesic in $S\left(T_{r}\right)$ and $S\left(S_{r}\right)$ corresponding to $\sigma_{r}$ is in both cases the imaginary axis (with the point at infinity included $)$. Therefore, $\sigma_{r}=S^{-1}(\{w \in \mathbb{C}: \operatorname{Re} w=$ $0\} \cup\{\infty\})=\{z \in \mathbb{C}:|z+1|=\sqrt{1+r}\}$.

To finish the proof we have to prove the following four facts:

1) $L_{T_{r}}\left(\sigma_{r}\right) \asymp \log (1 / r)$ as $r \longrightarrow 0$,

2) $L_{S_{r}}\left(\sigma_{r}\right) \asymp \log \log (1 / r)$ as $r \longrightarrow 0$,

3) $L_{T_{r}}\left(\sigma_{r}\right) \asymp 1 / \log r$ as $r \longrightarrow \infty$,

4) $L_{S_{r}}\left(\sigma_{r}\right) \asymp 1 / \log r$ as $r \longrightarrow \infty$.

1) follows as a direct consequence of Lemma 4.3 by observing that $\left\{n_{0}, n_{1}, \ldots, n_{\ell}\right\}=\{0,1,2, \ldots, N\}$ and $\ell=N \asymp \log (1 / t) \asymp \log (1 / r)$. Similarly, 2) follows also as a direct consequence of Lemma 4.3 since in this case $\ell \leq 3$.

3 ) is a well-known fact (see sections 1 and 2 of [LV, Chapter II], where 1) is also proved; recall that the product of the modulus of an annulus by the length of its simple closed geodesic is constant). 4) 
follows from 3), [BP, Theorem 1] and the fact that, as $r \geq 3$, the $\beta$ functions defined by (2.2) verify

$$
\beta_{T_{r}}(z)=\beta_{S_{r}}(z), \quad \text { for all } z \in \sigma_{r} .
$$

Lemma 4.5 has been proved.

Proof of TheOrem 2. Let us consider a border set of $\partial \Omega, B=$ $\left\{b_{1}, \ldots, b_{2 n}\right\}(n \geq 3)$. We have that $\hat{\mathbb{C}} \backslash\left(\left[b_{2 j}, b_{2 j+1}\right] \cup\left[b_{2 j+2}, b_{2 j-1}\right]\right) \subset$ $\Omega \subset \hat{\mathbb{C}} \backslash\left\{b_{2 j-1}, b_{2 j}, b_{2 j+1}, b_{2 j+2}\right\}$. Therefore, if we denote by $r$ the positive number

$$
r=r\left(\left\{b_{2 j-1}, b_{2 j}, b_{2 j+1}, b_{2 j+2}\right\}\right)
$$

Lemma 4.5 gives that

$$
\begin{aligned}
c_{2} \Phi_{2}(r) & \leq L_{\widehat{\mathbb{C}} \backslash\{0, r, \infty,-1\}}(\gamma(\{0, r, \infty,-1\})) \\
& =L_{\widehat{\mathbb{C}} \backslash\left\{b_{2 j-1}, b_{2 j}, b_{2 j+1}, b_{2 j+2}\right\}}\left(\gamma\left(\left\{b_{2 j-1}, b_{2 j}, b_{2 j+1}, b_{2 j+2}\right\}\right)\right) \\
& \leq L_{\Omega}\left(\gamma\left(\left\{b_{2 j-1}, b_{2 j}, b_{2 j+1}, b_{2 j+2}\right\}\right)\right),
\end{aligned}
$$

where we should remark that in the second line of the last display, $\gamma\left(\left\{b_{2 j-1}, b_{2 j}, b_{2 j+1}, b_{2 j+2}\right\}\right)$ refers to the geodesic in the domain $\hat{\mathbb{C}} \backslash$ $\left\{b_{2 j-1}, b_{2 j}, b_{2 j+1}, b_{2 j+2}\right\}$, but the same symbol in the third line refers to the geodesic in the domain $\Omega$.

Lemma 4.5 also gives that

$$
\begin{aligned}
L_{\Omega}(\gamma( & \left.\left.\left\{b_{2 j-1}, b_{2 j}, b_{2 j+1}, b_{2 j+2}\right\}\right)\right) \\
& \leq L_{\hat{\mathbb{C}} \backslash\left\{\left[b_{2 j}, b_{2 j+1}\right] \cup\left[b_{2 j+2}, b_{2 j-1}\right]\right\}}\left(\gamma\left(\left\{b_{2 j-1}, b_{2 j}, b_{2 j+1}, b_{2 j+2}\right\}\right)\right) \\
& =L_{\hat{\mathbb{C}} \backslash([-1,0] \cup[r, \infty])}(\gamma(\{0, r, \infty,-1\})) \\
& \leq c_{1} \Phi_{1}(r),
\end{aligned}
$$

where we should make a remark similar to the one in the last paragraph.

These inequalities and Lemma 4.2 prove Theorem 2 . 


\section{Length of geodesics and characterization of the HII-proper- ty in Denjoy domains.}

In order to state the characterization of the HII-property for Denjoy domains we need a good estimate of the length of the simple closed geodesic $\gamma(B)$ associated to any border set $B$ of $\partial \Omega$ with four points. This estimate, which is interesting by itself, is the statement of Theorem 4 .

Let us fix a number $0<a<1$ and denote by $D_{m}$ the closed set

$$
\begin{aligned}
D_{m}=D_{m}(B)=U(\{z \in \mathbb{C}: & a^{m+1} \leq|z+t(B)| \leq a^{m} \\
& \text { or } \left.\left.a^{m+1} \leq|z-t(B)| \leq a^{m}\right\}\right),
\end{aligned}
$$

$m \in \mathbb{N}$. The intersection of $D_{m}$ with the real axis is, in fact, a union of at most four closed intervals. Observe that the definition of $D_{m}$ above is consistent with the one in Lemma 4.3.

We need also to define the following natural number

$$
N=N_{B}:=\left[\frac{\log \frac{a}{t(B)}}{\log \frac{1}{a}}\right],
$$

where $[x]$ is the greatest natural number which is less or equal than $x$.

Theorem 4. Let $\Omega$ be a Denjoy domain, $0<a<1$ and $B=$ $\left\{b_{1}, b_{2}, b_{3}, b_{4}\right\}$ be a border set of $\partial \Omega$.

Let $n_{1}<n_{2}<\cdots<n_{\ell-1}$ be the list of the natural numbers in $(0, N)$ satisfying $D_{n_{j}} \cap \partial \Omega \neq \varnothing, n_{0}=0$ and $n_{\ell}=N$.

Then there exists a universal constant $0<r_{0}<1$ such that

$$
\begin{aligned}
L_{\Omega}(\gamma(B)) \asymp \Psi_{\Omega}(B) & \begin{array}{ll}
\frac{1}{\log r(B)}, & \text { if } r(B)>e, \\
1, & \text { if } r_{0}<r(B) \leq e, \\
\sum_{j=1}^{\ell}\left(1+\log \left(n_{j}-n_{j-1}\right)\right), & \text { if } r(B) \leq r_{0} .
\end{array}
\end{aligned}
$$

Here the constant in $\asymp$ depends only on a and neither on $\Omega$ nor $B$. 
Observe that Theorem 4 gives a general procedure to obtain the length of a symmetric simple closed geodesic in a Denjoy domain. This theorem is a useful tool in order to study the asymptotic behaviour of the length of geodesics in domains which depend on a parameter. Also, note that the condition of admissibility of $\Omega$ does not appear in the hypotheses.

In the proof of Theorem 5 we use Theorem 4 and some of the ingredients of the proof of Theorem 2. Theorem 4 allows to relate the previous ideas with euclidean conditions on the size of $\partial \Omega$; this is the most delicate part of our argument.

We start proving an analogue of Lemma 4.3 but now for the "authentic" geodesics. This result will be the basic tool in the proof of Theorem 4 .

Lemma 5.1. Let us fix a number $0<a<1$ and let $0<t<1$. For each natural number $m$ such that

$$
m \leq N=\left[\frac{\log \frac{a}{t}}{\log \frac{1}{a}}\right],
$$

let us consider the closed set

$D_{m}=D_{m}(t)=\left\{z \in \mathbb{C}: a^{m+1} \leq|z+t| \leq a^{m}\right.$ or $\left.a^{m+1} \leq|z-t| \leq a^{m}\right\}$.

Let $\Omega$ be a Denjoy domain such that $B=\{-t, t, 1,-1\} \subset \partial \Omega \subset$ $[-1,-t] \cup[t, 1]$.

Let $n_{1}<n_{2}<\cdots<n_{\ell-1}$ be all the natural numbers in $(0, N)$ satisfying $D_{n_{j}} \cap \partial \Omega \neq \varnothing, n_{0}=0$ and $n_{\ell}=N$.

Then we have that there exists a universal constant $0<t_{0}<1$ (the same constant that in Lemma 4.3) such that

$$
L_{\Omega}(\gamma(B)) \asymp \sum_{j=1}^{\ell}\left(1+\log \left(n_{j}-n_{j-1}\right)\right), \quad \text { for } 0<t \leq t_{0}
$$

Here the constant in $\asymp$ depends only on a and neither on $\Omega$ nor $t$.

The main ideas of the proof of this lemma are the following. First, we shall use a polarization argument (see below) in order to reduce our problem to some extremal cases (Lemma 5.2). Secondly, observe that 
we do not know where is the geodesic $\gamma(B)$. So, in order to obtain lower bounds for its length, we shall study the length of any curve in the same homotopy class in $\Omega$ by using again a "dyadic" argument (Lemma 5.3). We should remark that we have already upper bounds of the length of $\gamma(B)$ (Lemma 4.3).

In order to prove Lemma 5.1 it is convenient to introduce some concepts.

If $z$ is a complex number, we consider its symmetric point with respect to the imaginary axis $z^{\#}=-\bar{z}$, with $\infty^{\#}=\infty$. The symmetric $A^{\#}$ of a set $A \subset \hat{\mathbb{C}}$ is defined as $A^{\#}=\left\{z^{\#}: z \in A\right\}$. The positive and negative parts of $A$ are

$$
A^{+}=A \cap\{z: \operatorname{Re} z \geq 0\}, \quad A^{-}=A \cap\{z: \operatorname{Re} z \leq 0\} .
$$

Let us consider a domain $\Omega$ as in Lemma 5.1. The polarization $\Omega_{p}$ of the Denjoy domain $\Omega$ is defined as

$$
\Omega_{p}=\left(\Omega \cup \Omega^{\#}\right)^{+} \cup\left(\Omega \cap \Omega^{\#}\right)^{-}
$$

and the antisymmetric $\Omega_{a s}$ of the domain $\Omega$ as

$$
\Omega_{a s}=(\{z: \operatorname{Re} z \geq 0\} \backslash\{t, 1\}) \cup\left(\Omega \cap \Omega^{\#}\right)^{-} .
$$

Observe that $\left(\Omega_{p}\right)_{p}=\Omega_{p},\left(\Omega_{a s}\right)_{a s}=\Omega_{a s},\left(\Omega_{p}\right)_{a s}=\left(\Omega_{a s}\right)_{p}=\Omega_{a s}$ and $\Omega_{p} \subset \Omega_{a s}$.

The concept of polarization appeared in a paper by Wolontis [W], who proved results on the behavior of certain extremal lengths under polarization and also symmetrization results by repeated application of polarization. rem 9]

We shall need the following result about polarization [So, Theo-

$$
\lambda_{\Omega_{p}}(z) \leq \min \left\{\lambda_{\Omega}(z), \lambda_{\Omega}\left(z^{\#}\right)\right\}, \quad \text { if } \operatorname{Re} z \geq 0
$$

In particular, we have that

$$
\lambda_{\Omega_{p}}(z) \leq \lambda_{\Omega_{p}}\left(z^{\#}\right), \quad \lambda_{\Omega_{a s}}(z) \leq \lambda_{\Omega_{a s}}\left(z^{\#}\right), \quad \text { if } \operatorname{Re} z \geq 0 .
$$

This last result is well-known [M, Theorem 3].

The results concerning the Poincaré metric that appear in $[\mathrm{M}]$ and [So] use as symmetry axis the real axis instead of the imaginary one, 
but it is obvious (as Solynin comments in [So]) that the result is true for polarization with respect to any fixed straight line.

We can prove now

Lemma 5.2. In order to prove Lemma 5.1 it is enough to consider the sets $\Omega_{a s}$ instead of $\Omega$.

Proof. If $\sigma$ denotes the imaginary axis with the point at infinity, we have that

$$
L_{\Omega_{a s}}(\sigma) \asymp L_{\Omega_{p}}(\sigma) \asymp L_{\Omega}(\sigma), \quad \text { for } 0<t \leq t_{0},
$$

where $t_{0}$ is the constant in Lemma 4.3. This fact is a direct consequence of Lemma 4.3 , since the expression $\sum_{j=1}^{\ell}\left(1+\log \left(n_{j}-n_{j-1}\right)\right)$ is exactly the same for $\Omega_{a s}, \Omega_{p}$ and $\Omega$.

Let us consider now the simple closed geodesic $\gamma$, (respectively $\gamma_{p}, \gamma_{a s}$ ) in $\Omega$ (respectively $\Omega_{p}, \Omega_{a s}$ ) which is freely homotopic to $\sigma$. By the definition of geodesic it follows that

$$
L_{\Omega}(\gamma) \leq L_{\Omega}(\sigma), \quad L_{\Omega_{p}}\left(\gamma_{p}\right) \leq L_{\Omega_{p}}(\sigma), \quad L_{\Omega_{a s}}\left(\gamma_{a s}\right) \leq L_{\Omega_{a s}}(\sigma)
$$

We also have that $\lambda_{\Omega_{a s}}(z) \leq \lambda_{\Omega_{p}}(z)$ for all $z \in \Omega_{p}$, since $\Omega_{p} \subset \Omega_{a s}$. Therefore $L_{\Omega_{a s}}\left(\gamma_{a s}\right) \leq L_{\Omega_{p}}\left(\gamma_{p}\right)$.

In order to finish the proof of Lemma 5.2, it is enough to see that $L_{\Omega_{p}}\left(\gamma_{p}\right) \leq L_{\Omega}(\gamma)$.

Let us consider the curve $\tilde{\gamma}=\gamma^{+} \cup\left(\gamma^{-}\right)^{\#}$. Obviously, $\tilde{\gamma}$ is freely homotopic to $\gamma$ in $\Omega$. Therefore

$$
L_{\Omega_{p}}\left(\gamma_{p}\right) \leq L_{\Omega_{p}}(\tilde{\gamma}) \leq L_{\Omega}(\gamma)
$$

where the first inequality follows from the fact that $\tilde{\gamma}$ is also freely homotopic to $\gamma_{p}$ in $\Omega_{p}$, and we have the second one by (5.1).

This finishes the proof of Lemma 5.2.

Lemma 5.3. Let us fix a number $0<a<1$. Let $\Omega$ be a Denjoy domain such that $\{-1,-t, t, 1\} \subset \partial \Omega \subset[-1,-t] \cup[t, 1]$, with $0<t \leq t_{0}$, where $t_{0}$ is the constant in Lemma 4.3. Let us consider the antisymmetric set $\Omega_{a s}$ of $\Omega$. Let $\mu_{m}$ be a curve contained in $B_{m}=\{z \in \mathbb{C}: 0 \leq \operatorname{Re} z \leq$ $\left.(1+t) / 2, a^{m+1} \leq|z-t| \leq a^{m}\right\}$ which joins $S_{m}=\left\{z \in \mathbb{C}:|z-t|=a^{m}\right\}$ 
with $S_{m+1}$. Then, there exists a positive constant $c$, which only depends on a, such that if

$$
\begin{gathered}
\left(D_{m-k} \cup D_{m+k}\right) \cap \partial \Omega_{a s} \neq \varnothing, \\
\left(D_{m-k+1} \cup D_{m-k+2} \cup \cdots \cup D_{m-1}\right. \\
\left.\cup D_{m} \cup D_{m+1} \cup \cdots \cup D_{m+k-1}\right) \cap \partial \Omega_{a s}=\varnothing,
\end{gathered}
$$

then we have

$$
L_{\Omega_{a s}}\left(\mu_{m}\right) \geq \frac{c}{k+1},
$$

for $0 \leq k \leq \min \{m, N-m\}$ (obviously the second condition over $\partial \Omega_{a s}$ does not appear if $k=0$ ).

Proof. Let $\Omega_{a s}^{*}:=\Omega_{a s} \backslash\{\infty\}$; the computations in $\Omega_{a s}^{*}$ are easier than in $\Omega_{a s}$ because we can apply [BP, Theorem 1] since $\Omega_{a s}^{*} \subset \mathbb{C}$. We are going to find bounds for $\beta_{\Omega_{a s}^{*}}(z)$, in order to estimate $\lambda_{\Omega_{a s}^{*}}(z)$ for $z \in B_{m}$.

We have that $\delta_{\Omega_{a s}^{*}}(z)=|z-t|$ and (4.1) gives that

$$
t \leq a^{m+1} \leq|z-t|, \quad \text { for all } z \in B_{m} \text { and } m \leq N
$$

Let us consider a point $b \in\left(D_{m-k} \cup D_{m+k}\right) \cap \partial \Omega_{a s}^{*}$. We have four possibilities:

i) $b \in D_{m+k}$ and $a^{m+k+1} \leq|b+t| \leq a^{m+k}$,

ii) $b \in D_{m+k}$ and $a^{m+k+1} \leq|b-t| \leq a^{m+k}$,

iii) $b \in D_{m-k}$ and $a^{m-k+1} \leq|b+t| \leq a^{m-k}$,

iv) $b \in D_{m-k}$ and $a^{m-k+1} \leq|b-t| \leq a^{m-k}$.

We consider first the cases ii) and iv). The conditions which define these possibilities and (5.3) give that

$$
\frac{1}{a^{k-1}}=\frac{a^{m+1}}{a^{m+k}} \leq \frac{|z-t|}{|b-t|} \leq \frac{a^{m}}{a^{m+k+1}}=\frac{1}{a^{k+1}},
$$

in the case ii), and

$$
a^{k+1}=\frac{a^{m+1}}{a^{m-k}} \leq \frac{|z-t|}{|b-t|} \leq \frac{a^{m}}{a^{m-k+1}}=a^{k-1},
$$


in the case iv). In both cases, this implies that

$$
\beta_{\Omega_{a s}^{*}}(z) \leq(k+1) \log \frac{1}{a} .
$$

We consider now the cases i) and iii). If $b=1$ or $b=t$ we can take $-b$ instead of $b$ (since $-b$ also belongs to $\left.\left(D_{m-k} \cup D_{m+k}\right) \cap \partial \Omega_{a s}^{*}\right)$ and we are in the cases ii) or iv); obviously, $b \neq-t$. Therefore, without loss of generality we can assume that $b<-t$. In both possibilities i) and iii) we have that

$$
|b-t| \geq|b+t|
$$

In order to obtain upper bounds for $|b-t|$, we study separately the cases i) and iii).

In the case iii) we have that

$$
|b-t|=2 t+|b+t| \leq 2 a^{m+1}+a^{m-k} \leq 3 a^{m-k},
$$

and

$$
\frac{a^{k+1}}{3}=\frac{a^{m+1}}{3 a^{m-k}} \leq \frac{|z-t|}{|b-t|} \leq \frac{a^{m}}{a^{m-k+1}}=a^{k-1} .
$$

This fact implies that

$$
\beta_{\Omega_{a s}^{*}}(z) \leq \log 3+(k+1) \log \frac{1}{a} .
$$

In the case i) the condition $m+k \leq N$ gives that

$$
|b-t|=2 t+|b+t| \leq 2 a^{m+k+1}+a^{m+k} \leq 3 a^{m+k},
$$

and

$$
\frac{1}{3 a^{k-1}}=\frac{a^{m+1}}{3 a^{m+k}} \leq \frac{|z-t|}{|b-t|} \leq \frac{a^{m}}{a^{m+k+1}}=\frac{1}{a^{k+1}} .
$$

This fact implies that

$$
\beta_{\Omega_{a s}^{*}}(z) \leq \max \left\{\left|\log \frac{1}{3 a^{k-1}}\right|, \log \frac{1}{a^{k+1}}\right\} .
$$

Therefore, there is a constant $c_{1}$, only depending on $a$, such that

$$
\beta_{\Omega_{a s}^{*}}(z) \leq c_{1}(k+1) .
$$


Consequently, we have in any case

$$
\beta_{\Omega_{a s}^{*}}(z) \leq c_{2}(k+1)
$$

Therefore [BP, Theorem 1] gives that

$$
\lambda_{\Omega_{a s}^{*}}(z) \geq \frac{c_{3}}{|z-t|(k+1) \log \left(\frac{1}{a}\right)},
$$

and we deduce that

$$
\begin{aligned}
L_{\Omega_{a s}^{*}}\left(\mu_{m}\right) & =\int_{\mu_{m}} \lambda_{\Omega_{a s}^{*}}(z)|d z| \\
& \geq \int_{\mu_{m}} \frac{c_{3}|d z|}{|z-t|(k+1) \log \left(\frac{1}{a}\right)} \\
& \geq \int_{a^{m+1}}^{a^{m}} \frac{c_{3} d r}{r(k+1) \log \left(\frac{1}{a}\right)} \\
& =\frac{c_{3}}{k+1} .
\end{aligned}
$$

Observe that $|z-t| \leq a^{m} \leq 1$ and $t<1$. These facts imply that $|z|<2$. Lemma 4.4 (recall that we have chosen $\rho=2$ ) gives that

$$
L_{\Omega_{a s}}\left(\mu_{m}\right) \geq \frac{c}{k+1}, \quad \text { if } 0<t \leq t_{0} .
$$

This finishes the proof of Lemma 5.3.

Proof of Lemma 5.1. As Lemma 5.2 states, we only need to prove Lemma 5.1 for the domains $\Omega_{a s}$.

Let us consider any curve $\mu$ freely homotopic to $\gamma(B)$ in $\Omega_{a s}$.

We want to prove that there exists a positive constant $c_{1}$, which only depends on $a$, such that

$$
L_{\Omega_{a s}}(\mu) \geq c_{1} \sum_{j=1}^{\ell}\left(1+\log \left(n_{j}-n_{j-1}\right)\right) .
$$


If we prove this inequality, then Lemma 5.1 is true since $\gamma(B)$ is one of the curves $\mu$ above. The upper bound of $L_{\Omega_{a s}}(\mu)$ is a consequence of Lemma 4.3, since

$$
L_{\Omega_{a s}}(\gamma(B)) \leq L_{\Omega_{a s}}(\sigma) \leq c_{2} \sum_{j=1}^{\ell}\left(1+\log \left(n_{j}-n_{j-1}\right)\right)
$$

and the $n_{j}$ 's are the same for $\Omega_{a s}$ and $\Omega$.

Let us consider now the curve $\tilde{\mu}=\mu^{+} \cup\left(\mu^{-}\right)^{\#}$. Obviously $\tilde{\mu}$ is freely homotopic to $\mu$ in $\Omega_{a s}$, and (5.2) gives that $L_{\Omega_{a s}}(\tilde{\mu}) \leq L_{\Omega_{a s}}(\mu)$.

Let $\mu^{0}$ be a connected component of $\tilde{\mu}$ contained in $\{z: 0 \leq$ $\operatorname{Re} z \leq(1+t) / 2\}$ which joins the interval $[0, t)$ with $\{z: \operatorname{Re} z=$ $(1+t) / 2, \operatorname{Im} z \geq 0\}$. The curve $\mu^{0}$ meets the vertical line $\{z: \operatorname{Re} z=$ $(1+t) / 2\}$ at a point with the form $i b_{2}+(1+t) / 2$. We have that

$$
\left|\frac{1+t}{2}+i b_{2}-t\right| \geq \frac{1-t}{2} \geq \frac{1-t_{0}}{2} .
$$

If $m$ satisfies

$$
\frac{\log \frac{2}{1-t_{0}}}{\log \frac{1}{a}} \leq m \leq \frac{\log \frac{a}{t}}{\log \frac{1}{a}}
$$

then we have that $a^{m} \leq\left(1-t_{0}\right) / 2$ and $a^{m+1} \geq t$ and so $\mu_{m}=\mu^{0} \cap\{z$ : $\left.a^{m+1} \leq|z-t| \leq a^{m}\right\}$ joins $S_{m}=\left\{z:|z-t|=a^{m}\right\}$ with $S_{m+1}$. Therefore Lemma 5.3 and the same argument used at the end of the proof of Lemma 4.3 give that

$$
L_{\Omega_{a s}}(\mu) \geq L_{\Omega_{a s}}\left(\mu^{0}\right) \geq c_{1} \sum_{j=1}^{\ell}\left(1+\log \left(n_{j}-n_{j-1}\right)\right)
$$

since the terms in the last sum corresponding to

$$
0 \leq m \leq \frac{\log \frac{2}{1-t_{0}}}{\log \frac{1}{a}}
$$

have bounded length. 
Proof of Theorem 4. If we apply the Möbius transformation $U^{-1}$ (which preserves the hyperbolic metric) to $\Omega$ we obtain a new domain $\Omega^{\prime}$ with

$$
\{-1,-t, t, 1\} \subset \partial \Omega^{\prime} \subset[-1,-t] \cup[t, 1]
$$

Therefore, without loss of generality we can assume that $\Omega$ satisfies (5.5) and so

$$
D_{m}=\left\{z \in \mathbb{C}: a^{m+1} \leq|z+t| \leq a^{m} \text { or } a^{m+1} \leq|z-t| \leq a^{m}\right\} .
$$

Let $\gamma$ be the simple closed geodesic in $\Omega$ given by $\gamma=\gamma(\{-t, t, 1,-1\})$.

Let us consider first the case $0<t \leq t_{0}$. Lemma 5.1 gives that

$$
L_{\Omega}(\gamma) \asymp \sum_{j=1}^{\ell}\left(1+\log \left(n_{j}-n_{j-1}\right)\right) .
$$

For $t_{0} \leq t<1$, observe that $\Omega_{1}=\hat{\mathbb{C}} \backslash([-1,-t] \cup[t, 1]) \subset \Omega \subset \Omega_{2}=$ $\hat{\mathbb{C}} \backslash\{-1,-t, t, 1\}$. Then we have that

$$
\begin{aligned}
& \lambda_{\Omega_{1}}(z) \geq \lambda_{\Omega}(z), \quad \text { for all } z \in \Omega_{1}, \\
& \lambda_{\Omega}(z) \geq \lambda_{\Omega_{2}}(z), \quad \text { for all } z \in \Omega,
\end{aligned}
$$

and consequently Lemma 4.5 gives that

$$
\Phi_{1}(r) \asymp L_{\Omega_{1}}(\sigma) \geq L_{\Omega}(\gamma) \geq L_{\Omega_{2}}(\sigma) \asymp \Phi_{2}(r), \quad \text { with } r=\frac{4 t}{(1-t)^{2}},
$$

and we have that

$$
\Phi_{1}(r) \asymp \Phi_{2}(r) \asymp \begin{cases}\frac{1}{\log r}, & \text { if } r>e, \\ 1, & \text { if } r_{0} \leq r \leq e,\end{cases}
$$

with $r_{0}=4 t_{0} /\left(1-t_{0}\right)^{2}$. Here the constant in $\asymp$ depends only on $a$ but neither on $\Omega$ nor $r$. This finishes the proof of Theorem 4 .

Theorem 5. Let $\Omega$ be a Denjoy domain, let $I$ be the set of isolated points of $\partial \Omega$ and let $\Omega_{0}=\Omega \cup I$. Then, $\Omega$ has HII if and only if $\Omega$ 
is admissible and there exists a positive constant $c$ such that for any border set of $\partial \Omega_{0}, B=\left\{b_{1}, \ldots, b_{2 n}\right\}$ with $n \geq 3$, we have that

$$
\frac{1}{n} \sum_{j=1}^{n} \Psi_{\Omega_{0}}\left(\left\{b_{2 j-1}, b_{2 j}, b_{2 j+1}, b_{2 j+2}\right\}\right)>c .
$$

Proof of TheOrem 5. If $\partial \Omega_{0}$ has isolated points, then $\Omega$ is not admissible and Theorem 1 gives that $\Omega$ has not HII. Let us assume now that $\partial \Omega_{0}$ has not isolated points. Theorem 1 reduces the proof of Theorem 5 to the following:

$\Omega_{0}$ has HII if and only if there exists a positive constant $c$ such that for any border set of $\partial \Omega_{0}, B=\left\{b_{1}, \ldots, b_{2 n}\right\}$ with $n \geq 3$, we have that

$$
\frac{1}{n} \sum_{j=1}^{n} \Psi_{\Omega_{0}}\left(\left\{b_{2 j-1}, b_{2 j}, b_{2 j+1}, b_{2 j+2}\right\}\right)>c .
$$

This fact is a consequence of Lemma 4.2 and Theorem 4 .

\section{Collars and balls.}

Let $\mathcal{R}$ be a hyperbolic Riemann surface with a puncture $p$. A collar in $\mathcal{R}$ about $p$ is a doubly connected domain in $\mathcal{R}$ "bounded" by $p$ and a Jordan curve (called the boundary curve of the collar) orthogonal to the pencil of geodesics emanating from $p$. It is well known that the length of the boundary curve is equal to the area of the collar.

A collar in $\mathcal{R}$ about $p$ of area $\alpha$ will be called an $\alpha$-collar and it will be denoted by $C_{\mathcal{R}}(p, \alpha)$. A theorem of Shimizu [S] gives that for every puncture in any hyperbolic Riemann surface, there exists an $\alpha$-collar for every $0<\alpha \leq 1$ (see also [K, p. 60-61]).

Next, we will prove a relationship (involving universal constants) between collars in $\mathcal{R}$ and balls in $\mathcal{R} \cup\{p\}$.

Proposition 1. Let $\mathcal{S}$ be a hyperbolic Riemann surface and let $\left\{B_{\mathcal{S}}\left(p, r_{0}\right)\right\}_{p \in I}$ be a family of simply connected and pairwise disjoint balls. Let us denote by $\mathcal{R}$ the Riemann surface $\mathcal{R}=\mathcal{S} \backslash$ I. Let $k=4.76$ and $K=e^{k}$. 
a) We have that

$$
C_{\mathcal{R}}\left(p, \frac{2 \pi}{k-\log \left(1-e^{-r}\right)}\right) \subset B_{\mathcal{S}}(p, r)^{*}
$$

for $p \in I$ and

$$
0<r<\min \left\{\log \frac{1}{1-K e^{-2 \pi}}, \log \frac{2}{1+e^{-2 r_{0}}}\right\}
$$

b) We have that

$$
B_{\mathcal{S}}(p, r)^{*} \subset C_{\mathcal{R}}\left(p, \frac{2 \pi}{\log \left(1-e^{-2 r_{0}}\right)-\log \left(e^{r}-1\right)}\right)
$$

for $p \in I$ and

$$
0<r<\log \left(1+\left(1-e^{-2 r_{0}}\right) e^{-2 \pi}\right) .
$$

Observe that, in both cases, the conditions on $r$ imply that $0<$ $r<r_{0}$.

Proof. Let $F: \mathbb{U} \longrightarrow \mathcal{S}$ be a universal covering map and $J=F^{-1}(I)$. The balls in $\left\{B_{\mathbb{U}}\left(z, r_{0}\right)\right\}_{z \in J}=\left\{F^{-1}\left(B_{\mathcal{S}}\left(p, r_{0}\right)\right)\right\}_{p \in I}$ are obviously simply connected (every ball in $\mathbb{U}$ is simply connected). We also remark that these balls are pairwise disjoint. If we have that for some $z, w \in J$

$$
B_{\mathbb{U}}\left(z, r_{0}\right) \cap B_{\mathbb{U}}\left(w, r_{0}\right) \neq \varnothing,
$$

this implies that $B_{\mathcal{S}}\left(F(z), r_{0}\right.$ ) is not simply connected (if $F(z)=F(w)$ ) or $B_{\mathcal{S}}\left(F(z), r_{0}\right) \cap B_{\mathcal{S}}\left(F(w), r_{0}\right) \neq \varnothing$ (if $F(z) \neq F(w)$ ), and both conclusions contradict the hypothesis on $\left\{B_{\mathcal{S}}\left(p, r_{0}\right)\right\}_{p \in I}$.

Since

$$
F\left(B_{\mathbb{U}}(z, r)\right)=B_{\mathcal{S}}(F(z), r), \quad \text { for } z \in J, 0<r \leq r_{0},
$$

and

$$
F\left(C_{\mathbb{U} \backslash J}(z, \alpha)\right)=C_{\mathcal{S} \backslash I}(F(z), \alpha), \quad \text { for } z \in J, 0<\alpha<1,
$$

we have that Proposition 1 is true for all hyperbolic Riemann surface $\mathcal{S}$ if and only if it is true for the case $\mathcal{S}=\mathbb{U}$ (with the same constants). 
Therefore, without loss of generality we can assume that $\mathcal{S}=\mathbb{U}$. Let $\mathbb{V}$ be the Riemann surface $\mathbb{V}=\mathbb{U} \backslash I$.

In the following we need a precise version of (2.3). It is well known that if $\Omega \subset \mathbb{C}$ is a hyperbolic plane domain then

$$
\lambda_{\Omega}(z) \geq \frac{1}{\delta_{\Omega}(z)\left(4.76+\beta_{\Omega}(z)\right)}, \quad \text { for } z \in \Omega .
$$

Lemma 6.1. Let $r>0, z_{1}, z_{2} \in \mathbb{U}$. If $d_{\mathbb{U}}\left(z_{1}, z_{2}\right) \geq 2 r$ and $z \in$ $B_{\mathbb{U}}\left(z_{1}, a(r)\right)$, we have that

$$
\left|z-z_{1}\right|<\left|z-z_{2}\right|
$$

where

$$
a(r)=\log \frac{2}{1+e^{-2 r}}
$$

PROOF. Since this statement is invariant under conformal automorphisms of $\mathbb{U}$, we can assume without loss of generality that $z_{1}=i$ and $d_{\mathbb{U}}\left(i, z_{2}\right)=2 r$.

A computation gives that Lemma 6.1 is true if (6.2) holds for $z_{2}=$ $i e^{-2 r}$ and $z$ belongs to the segment joining $i e^{-a(r)}$ with $i$ (this is the worse case) and this follows from our election of $a(r)$.

Using (6.1) and Lemma 6.1 we can prove the following result.

Lemma 6.2. Let $\left\{B_{\mathbb{U}}\left(p, r_{0}\right)\right\}_{p \in I}$ be a family of pairwise disjoint balls. Then we have, for $p \in I$, that

$$
\lambda_{\mathbb{V}}(z) \leq \lambda_{B\left(p,\left(1-e^{-2 r_{0}}\right) \operatorname{Im} p\right)^{*}}(z),
$$

for $z \in B\left(p,\left(1-e^{-2 r_{0}}\right) \operatorname{Im} p\right)^{*}$, and

$$
\lambda_{B(p, K \operatorname{Im} p)^{*}}(z) \leq \lambda_{\mathbb{V}}(z), \quad \text { for } \quad z \in B_{\mathbb{U}}\left(p, a\left(r_{0}\right)\right),
$$

where $a(r)$ is the function defined in Lemma 6.1 .

Proof. The following relationship between hyperbolic and euclidean balls is well-known.

$$
B_{\mathbb{U}}(x+i y, r)=B(x+i y \cosh r, y \sinh r), \quad \text { for } x \in \mathbb{R}, y, r>0 .
$$


This implies that

$$
B\left(z,\left(1-e^{-r}\right) \operatorname{Im} z\right) \subset B_{\mathbb{U}}(z, r) \subset B\left(z,\left(e^{r}-1\right) \operatorname{Im} z\right),
$$

for $z \in \mathbb{U}, r>0$. We deduce that

$$
B\left(p,\left(1-e^{-2 r_{0}}\right) \operatorname{Im} p\right) \subset B_{\mathbb{U}}\left(p, 2 r_{0}\right), \quad \text { for } p \in I .
$$

Since $d_{\mathbb{U}}(p, q) \geq 2 r_{0}$ for all $p, q \in I, p \neq q$, we have that $B(p,(1-$ $\left.\left.e^{-2 r_{0}}\right) \operatorname{Im} p\right)^{*} \subset \mathbb{V}$. This implies (6.3).

Using again that $d_{\mathbb{U}}(p, q) \geq 2 r_{0}$ for all $p, q \in I, p \neq q$, and Lemma 6.1 we deduce that

$$
|z-p|<|z-q|, \quad \text { for } z \in B_{\mathbb{U}}\left(p, a\left(r_{0}\right)\right) \text {. }
$$

A computation gives that

$$
|z-p| \leq \operatorname{Im} z, \quad \text { for } z \in B_{\mathbb{U}}\left(p, a\left(r_{0}\right)\right),
$$

since $e^{a\left(r_{0}\right)}<2$. Hence, (6.6) and (6.7) imply that

$$
\delta_{\mathbb{V}}(z)=|z-p|, \quad \text { for } z \in B_{\mathbb{U}}\left(p, a\left(r_{0}\right)\right) \text {. }
$$

Consequently,

$$
\beta_{\mathbb{V}}(z) \leq \min \left\{|\log | \frac{z-p}{w-p}||: w \in \partial \mathbb{V}\right\} \leq \log \frac{\operatorname{Im} p}{|z-p|},
$$

since $|z-p| \leq \operatorname{Im} p$, for $z \in B_{\mathbb{U}}\left(p, a\left(r_{0}\right)\right.$ ) (to see this it is enough to change the roles of $z$ and $p$ in (6.7)).

Now, (6.1) and (6.8) imply that

$$
\lambda_{\mathbb{V}}(z) \geq \frac{1}{|z-p| \log \frac{K \operatorname{Im} p}{|z-p|}}, \quad \text { for } z \in B_{\mathbb{U}}\left(p, a\left(r_{0}\right)\right) .
$$

This inequality and the well known fact

$$
\lambda_{B(w, c)^{*}}(z)=\frac{1}{|z-w| \log \frac{c}{|z-w|}}, \quad \text { for } z \in B(w, c)^{*}
$$


give (6.4). This finishes the proof of Lemma 6.2.

Next we will prove Proposition 1, part a). First of all we observe that $K e^{-2 \pi}<1$, since $k<2 \pi$. Secondly, the condition $r<-\log (1-$ $\left.K e^{-2 \pi}\right)$ implies that

$$
0<\frac{2 \pi}{k-\log \left(1-e^{-r}\right)}<1
$$

and then we can assure that there exists the collar in $\mathcal{R}$ [K, p. 60-61].

On the other hand, (6.4) and (6.5) give, for $p \in I$, that

$$
\lambda_{B(p, K \operatorname{Im} p)^{*}}(z) \leq \lambda_{\mathbb{V}}(z),
$$

for $z \in B\left(p,\left(1-e^{-a\left(r_{0}\right)}\right) \operatorname{Im} p\right)^{*} \subset B_{\mathbb{U}}\left(p, a\left(r_{0}\right)\right)^{*}$. A straightforward computation shows that, for $w \in \mathbb{C}$ and $\rho>0$,

$$
\begin{aligned}
& C_{B(w, \rho)^{*}}(w, \alpha)=B\left(w, \rho e^{-2 \pi / \alpha}\right)^{*}, \quad \text { for } \alpha>0, \\
& B(w, r)^{*}=C_{B(w, \rho)^{*}}\left(w, \frac{2 \pi}{\log \rho-\log r}\right), \quad \text { for } 0<r<\rho .
\end{aligned}
$$

Therefore (6.9) and (6.10) imply that

$$
C_{\mathbb{V}}(p, \alpha) \subset C_{B(p, K \operatorname{Im} p)^{*}}(p, \alpha)=B\left(p, K e^{-2 \pi / \alpha} \operatorname{Im} p\right)^{*},
$$

if

$$
B\left(p, K e^{-2 \pi / \alpha} \operatorname{Im} p\right)^{*} \subset B\left(p,\left(1-e^{-a\left(r_{0}\right)}\right) \operatorname{Im} p\right)^{*} \subset B_{\mathbb{U}}\left(p, a\left(r_{0}\right)\right)^{*}
$$

If we choose

$$
\alpha=\frac{2 \pi}{k-\log \left(1-e^{-r}\right)},
$$

we obtain that

$$
\begin{aligned}
C_{\mathbb{V}}\left(p, \frac{2 \pi}{k-\log \left(1-e^{-r}\right)}\right) & \subset B\left(p, K e^{-2 \pi / \alpha} \operatorname{Im} p\right)^{*} \\
& =B\left(p,\left(1-e^{-r}\right) \operatorname{Im} p\right)^{*} \\
& \subset B_{\mathbb{U}}(p, r)^{*} \\
& \subset B_{\mathbb{U}}\left(p, a\left(r_{0}\right)\right)^{*},
\end{aligned}
$$


if

$$
r \leq a\left(r_{0}\right)=\log \frac{2}{1+e^{-2 r_{0}}} .
$$

This finishes the proof of Proposition 1, part a).

Finally, to prove part b), observe that the condition $r<\log (1+$ $\left.\left(1-e^{-2 r_{0}}\right) e^{-2 \pi}\right)$ implies that

$$
0<\frac{2 \pi}{\log \left(1-e^{-2 r_{0}}\right)-\log \left(e^{r}-1\right)}<1
$$

and then, as above, we can assure that there exists the collar in $\mathcal{R}$.

Now, for any $p \in I,(6.10)$ and (6.3) give that

$$
B\left(p,\left(1-e^{-2 r_{0}}\right) e^{-2 \pi / \alpha} \operatorname{Im} p\right)^{*}=C_{B\left(p,\left(1-e^{-2 r_{0}}\right) \operatorname{Im} p\right)^{*}}(p, \alpha) \subset C_{\mathbb{V}}(p, \alpha),
$$

for $0<\alpha<1$. In particular, if we choose

$$
\alpha=\frac{2 \pi}{\log \left(1-e^{-2 r_{0}}\right)-\log \left(e^{r}-1\right)}
$$

we obtain that

$$
B\left(p,\left(e^{r}-1\right) \operatorname{Im} p\right)^{*} \subset C_{\mathbb{V}}\left(p, \frac{2 \pi}{\log \left(1-e^{-2 r_{0}}\right)-\log \left(e^{r}-1\right)}\right) .
$$

Therefore (6.5) gives that

$$
B_{\mathbb{U}}(p, r)^{*} \subset C_{\mathbb{V}}\left(p, \frac{2 \pi}{\log \left(1-e^{-2 r_{0}}\right)-\log \left(e^{r}-1\right)}\right) .
$$

This finishes the proof of Proposition 1.

We define a generalized collar in a hyperbolic Riemann surface $\mathcal{R}$ about a puncture $p$ as a domain (not necessarily doubly connected) in $\mathcal{R}$ "bounded" by $p$ and a finite number of curves (if the collar is not equal to $\mathcal{R}$ ) orthogonal to the pencil of geodesics emanating from $p$. Observe that if $\mathcal{R}$ is a punctured compact surface (with only a puncture $p$ ), when the collar "grows" it is eventually equal to $\mathcal{R}$ and then there are not such boundary curves.

In the punctured disk, $\mathcal{R}=B(z, r)^{*}$ we have that

$$
d_{\mathcal{R}}\left(\partial C_{\mathcal{R}}\left(z, \alpha_{1}\right), \partial C_{\mathcal{R}}\left(z, \alpha_{2}\right)\right)=\left|\log \frac{\alpha_{1}}{\alpha_{2}}\right| .
$$


Then, we can define for $\alpha \geq 1$ the generalized $\alpha$-collar in $\mathcal{R}$ about $p$ as the set

$$
C_{\mathcal{R}}(p, \alpha)=C_{\mathcal{R}}\left(p, \frac{1}{2}\right) \cup\left\{q \in \mathcal{R}: d_{\mathcal{R}}\left(q, \partial C_{\mathcal{R}}\left(p, \frac{1}{2}\right)\right)<\log (2 \alpha)\right\} .
$$

Obviously this definition coincides with the original one if there exists the $\alpha$-collar. The number $1 / 2$ can be changed for any number $0<\eta<1$, if $\log (2 \alpha)$ is substituted by $\log (\alpha / \eta)$.

If $\mathcal{R}$ is not a punctured disk, it is obvious that there exists an $\alpha_{0}$ such that there is an $\alpha$-collar only for $0<\alpha \leq \alpha_{0}$. However there always are generalized $\alpha$-collars.

With this definition we can extend part b) of Proposition 1.

Corollary 3. Let $\mathcal{S}$ be a hyperbolic Riemann surface and let $\left\{B_{\mathcal{S}}\left(p, r_{0}\right)\right\}_{p \in I}$ be a family of simply connected and pairwise disjoint balls. Let us denote by $\mathcal{R}$ the Riemann surface $\mathcal{R}=\mathcal{S} \backslash$ I. If we denote the generalized $\alpha$-collar by $C_{\mathcal{R}}(p, \alpha)$, then we have that

$$
B_{\mathcal{S}}(p, r)^{*} \subset C_{\mathcal{R}}\left(p, \frac{2 \pi}{\log \left(1-e^{-2 r_{0}}\right)-\log \left(e^{r}-1\right)}\right),
$$

for $p \in I$ and

$$
0<r<\min \left\{\log \left(2-e^{-2 r_{0}}\right), r_{0}\right\}
$$

The proof of Corollary 3 is the same as the proof of Proposition 1 , part b). We do not need now the condition $\alpha<1$ but we also need $\alpha>0$; the condition on $r$ guarantees this fact.

A computation and (6.10) give that

$$
B_{B(w, \rho)}(w, r)^{*}=B\left(w, \rho \tanh \left(\frac{r}{2}\right)\right)^{*}=C_{B(w, \rho)^{*}}\left(w, \frac{2 \pi}{\log \operatorname{cotanh}\left(\frac{r}{2}\right)}\right)
$$

for $w \in \mathbb{C}$ and $\rho, r>0$.

We want to remark that Proposition 1 is sharp for $r \rightarrow 0$ in the following sense

$$
\lim _{r \rightarrow 0^{+}} \frac{\frac{2 \pi}{\frac{k-\log \left(1-e^{-r}\right)}{2 \pi}}}{-\log \tanh \left(\frac{r}{2}\right)}=\lim _{r \rightarrow 0^{+}} \frac{\frac{2 \pi}{\log \left(1-e^{-2 r_{0}}\right)-\log \left(e^{r}-1\right)}}{\frac{2 \pi}{-\log \tanh \left(\frac{r}{2}\right)}}=1 .
$$


Proposition 1 also gives the following result.

Corollary 4. Let $\mathcal{S}$ be a hyperbolic Riemann surface and let $\left\{B_{\mathcal{S}}\left(p, r_{0}\right)\right\}_{p \in I}$ be a family of simply connected and pairwise disjoint balls. Let us denote by $\mathcal{R}$ the Riemann surface $\mathcal{R}=\mathcal{S} \backslash I$. Let $k=4.76$ and $K=e^{k}$.

a) We have that

$$
C_{\mathcal{R}}(p, \alpha) \subset B_{\mathcal{S}}\left(p, \log \frac{1}{1-K e^{-2 \pi / \alpha}}\right)^{*}
$$

for $p \in I, 0<\alpha<1$ and

$$
\alpha \leq \frac{2 \pi}{k+\log \frac{2}{1-e^{-2 r_{0}}}} .
$$

b) If we denote the generalized $\alpha$-collar by $C_{\mathcal{R}}(p, \alpha)$, then we have that

$$
B_{\mathcal{S}}\left(p, \log \left(1+\left(1-e^{-2 r_{0}}\right) e^{-2 \pi / \alpha}\right)\right)^{*} \subset C_{\mathcal{R}}(p, \alpha),
$$

for $p \in I$ and

$$
0<\alpha \leq \frac{2 \pi}{\log \left(1+e^{-r_{0}}\right)-r_{0}}
$$

\section{Further results.}

We will generalize theorems 3 and 5 in this section. To do this, we shall comment some remarks:

1 ) If the set $I$ in theorems 3 and 5 is not contained in $\hat{\mathbb{R}}$, these theorems are also true since Theorem 1 is a general result about hyperbolic Riemann surfaces.

2) If $\partial \Omega_{0}$ is contained in a quasicircle $Q$ (the image of a straight line by a quasiconformal mapping of the Riemann sphere onto itself) our characterization of the HII-property for Denjoy domains can be yet applied (if we know the quasiconformal mapping which applies $\hat{\mathbb{R}}$ in $Q$ ) since the HII-property is preserved by quasiconformal mappings [FR, Theorem 1].

We can define in an obvious way a border set of a closed subset of a quasicircle. In this context we can generalize Theorem 3 . 
Theorem 6. Let $\Omega_{0}$ be a hyperbolic plane domain whose boundary is contained in a quasicircle and has not isolated points, let I be a strongly uniformly separated set in $\Omega_{0}$, and let $\Omega=\Omega_{0} \backslash I$. Then

a) If $\Omega$ has HII, then there exists a positive constant $c$ such that for any border set of $\partial \Omega_{0}, B=\left\{b_{1}, \ldots, b_{2 n}\right\}$ with $n \geq 3$, we have that

$$
\frac{1}{n} \sum_{j=1}^{n} \Phi_{1}\left(r\left(\left\{b_{2 j-1}, b_{2 j}, b_{2 j+1}, b_{2 j+2}\right\}\right)\right)>c .
$$

b) If there exists a positive constant $c$ such that for any border set of $\partial \Omega_{0}, B=\left\{b_{1}, \ldots, b_{2 n}\right\}$ with $n \geq 3$, we have that

$$
\frac{1}{n} \sum_{j=1}^{n} \Phi_{2}\left(r\left(\left\{b_{2 j-1}, b_{2 j}, b_{2 j+1}, b_{2 j+2}\right\}\right)\right)>c,
$$

then $\Omega$ has HII.

Observe that Theorem 6 follows directly from Theorem 3, [FR, Theorem 1] and the following facts: a) a quasiconformal map quasipreserves cross ratios; b) $\Phi_{i}(s) \asymp \Phi_{i}(r)$ for $s \asymp r$, with $0<r<\infty$ and $i=1,2$.

Theorem 6 gives a necessary and a sufficient condition for $\Omega$ to have HII. We shall improve this result in the remainder of the section.

If $\partial \Omega_{0}$ is contained in a finite union of quasicircles, we can also characterize the HII-property of $\Omega$ in many cases. We give now the details:

Let $\left\{E_{j}\right\}_{j=1}^{n}$ be a collection of pairwise disjoint closed subsets of $\hat{\mathbb{C}}$ such that each $E_{j}$ is contained in a quasicircle and $\Omega_{0}=\hat{\mathbb{C}} \backslash \cup_{j} E_{j}$ is connected. Let $I$ be a strongly uniformly separated set in $\Omega_{0}$ and let $\Omega=\Omega_{0} \backslash I$. A necessary and sufficient condition for $\Omega$ to have HII is that each $\hat{\mathbb{C}} \backslash E_{j}$ has HII (see Theorem 8 below). By using remark 2) or Theorem 6 as a test, we can verify if each one of these last domains has HII or not.

Although we are interested in plane domains and closed subsets of quasicircles, many results in this section are true for general Riemann surfaces instead of $\hat{\mathbb{C}}$ and general closed sets $E_{j}$. We start with some definitions. 
Definition. Let $\mathcal{S}$ be a Riemann surface and $\varepsilon>0$. Let $E_{1}, E_{2}$ be two closed disjoint subsets of $\mathcal{S}$. We say that $E_{1}$ and $E_{2}$ are weakly $\varepsilon$-separated in $\mathcal{S}$ if $\mathcal{S}_{1}=\mathcal{S} \backslash E_{1}, \mathcal{S}_{2}=\mathcal{S} \backslash E_{2}$ are (connected) hyperbolic Riemann surfaces and the two following subsets are disjoint:

$$
\begin{aligned}
& E_{1, \varepsilon}=\left\{q \in \mathcal{S}_{2}: d_{\mathcal{S}_{2}}\left(q, E_{1}\right)<2 \varepsilon\right\}, \\
& E_{2, \varepsilon}=\left\{q \in \mathcal{S}_{1}: d_{\mathcal{S}_{1}}\left(q, E_{2}\right)<2 \varepsilon\right\} .
\end{aligned}
$$

We say that $E_{1}$ and $E_{2}$ are weakly separated in $\mathcal{S}$ if they are weakly $\varepsilon$-separated in $\mathcal{S}$ for some $\varepsilon>0$.

We say that the closed sets $E_{1}, E_{2}, \ldots, E_{n}$ are weakly separated in $\mathcal{S}$ if the $n-1$ pairs of sets $\left(E_{1}, E_{2}\right),\left(E_{1} \cup E_{2}, E_{3}\right), \ldots,\left(E_{1} \cup E_{2} \cup \cdots \cup\right.$ $\left.E_{n-1}, E_{n}\right)$ are weakly separated in $\mathcal{S}$.

REMARK 1. It is clear that if $E_{1}, E_{2}$ are disjoint closed subsets of $\mathcal{S}, E_{2}$ is compact and $\mathcal{S} \backslash\left(E_{1} \cup E_{2}\right)$ is connected, then $E_{1}, E_{2}$ are weakly separated in $\mathcal{S}$. It is also clear that if $E_{1}, E_{2}, \ldots, E_{n}$ are pairwise disjoint compact subsets of $\mathcal{S}$ and $\mathcal{S} \backslash \cup_{j=1}^{n} E_{j}$ is connected, then $E_{1}, E_{2}, \ldots, E_{n}$ are weakly separated in $\mathcal{S}$.

REMARK 2. If $E_{1}, E_{2}$ are disjoint closed subsets of a plane domain $\Omega$, it is possible that they are not weakly separated in $\Omega$. Let $\Omega$ be the plane domain $\Omega=\mathbb{C} \backslash\{0\}$. Let us consider as $E_{1}$ a sequence $\left\{x_{n}\right\}$ of real numbers decreasing to 0 . Let $E_{2}$ be a sequence $\left\{y_{n}\right\}$ such that:

a) $0<x_{n+1}<y_{n}<x_{n}$,

b) $\lim _{n \rightarrow \infty}\left(x_{n}-y_{n}\right) /\left(y_{n}-x_{n+1}\right)=\lim _{n \rightarrow \infty}\left(x_{n}-y_{n}\right) /\left(y_{n-1}-x_{n}\right)=$ 0 .

Then $E_{1}, E_{2}$ are not weakly separated in $\mathbb{C} \backslash\{0\}$.

REMARK 3. Let $E_{1}, E_{2}$ be closed sets in a domain $\Omega \subset \mathbb{C} \backslash\left\{z_{0}\right\}$. Let us assume that there is a positive constant $\delta_{0}$ such that

$$
\left|z_{1}-z_{2}\right| \geq \delta_{0}\left|z_{1}-z_{0}\right|, \quad \text { for all } z_{1} \in E_{1}, z_{2} \in E_{2} .
$$

Then $E_{1}, E_{2}$ are weakly separated in $\Omega$.

Proof OF REMARK 3. Without loss of generality we can suppose $z_{0}=0$. For $w \in \mathbb{C} \backslash\{0,1\}$, we define the function

$$
e(w):=\max \left\{\varepsilon>0: B_{\mathbb{C} \backslash\{0,1\}}(w, \varepsilon) \cap B_{\mathbb{C} \backslash\{0, w\}}(1, \varepsilon)=\varnothing\right\} .
$$


Observe that $\partial B_{\mathbb{C} \backslash\{0,1\}}(w, \varepsilon)$ and $\partial B_{\mathbb{C} \backslash\{0, w\}}(1, \varepsilon)$ vary continuously with $w$, since

$$
\lambda_{\mathbb{C} \backslash\{0, w\}}(z)=\lambda_{\mathbb{C} \backslash\{0,1\}}\left(\frac{z}{w}\right) \frac{1}{|w|}
$$

is a real analytic function on $w$.

Therefore, $e$ is a continuous function $e: \mathbb{C} \backslash\{0,1\} \longrightarrow(0, \infty)$. On the other hand, we can deduce of (6.1) that

$$
\lambda_{\mathbb{C} \backslash\{0,1\}}(z) \geq \frac{1}{|z|(k+|\log | z||)}, \quad \text { for } z \in \mathbb{C} \backslash\{0,1\},
$$

where $k=4.76$ is the constant in Section 6 . This is a bad estimate if $z$ is near 1 , but it is good for $z$ in a neighborhood of 0 or $\infty$. This inequality gives

$$
B_{\mathbb{C} \backslash\{0,1\}}(w, \varepsilon) \subset\left\{|z|>\exp \left((k+\log |w|) e^{-\varepsilon}-k\right)\right\},
$$

and consequently,

$$
B_{\mathbb{C} \backslash\{0, w\}}(1, \varepsilon) \subset\left\{|z|<|w| \exp \left(k-(k+\log |w|) e^{-\varepsilon}\right)\right\},
$$

for

$$
|w|>1 \quad \text { and } \quad 0<\varepsilon \leq \log \frac{k+\log |w|}{k} .
$$

Therefore, $B_{\mathbb{C} \backslash\{0,1\}}(w, \varepsilon) \cap B_{\mathbb{C} \backslash\{0, w\}}(1, \varepsilon)=\varnothing$ for

$$
|w|>1 \quad \text { and } \quad 0<\varepsilon \leq \log \frac{k+\log |w|}{k+\frac{1}{2} \log |w|} .
$$

Then, for any $M>1$, there is a positive constant $c_{0}$ such that $e(w) \geq c_{0}$ if $|w| \geq M$.

Observe that $e(1 / w)=e(w)$ since the conformal map $T(z)=1 / z$ is an isometry of $\mathbb{C} \backslash\{0,1\}$ onto itself. Consequently, $e(w) \geq c_{0}$ if $|w| \leq 1 / M$. These facts imply that, for any $\delta>0$, there exists $\varepsilon>0$ such that $e(w) \geq \varepsilon$ if $|w-1| \geq \delta$.

For $z_{1}, z_{2} \in \mathbb{C} \backslash\{0\}$ with $z_{1} \neq z_{2}$, we define now the function

$$
E\left(z_{1}, z_{2}\right):=\max \left\{\varepsilon>0: B_{\mathbb{C} \backslash\left\{0, z_{1}\right\}}\left(z_{2}, \varepsilon\right) \cap B_{\mathbb{C} \backslash\left\{0, z_{2}\right\}}\left(z_{1}, \varepsilon\right)=\varnothing\right\} .
$$

It is clear that

$$
E\left(z_{1}, z_{2}\right)=E\left(1, \frac{z_{2}}{z_{1}}\right)=e\left(\frac{z_{2}}{z_{1}}\right)
$$


The hypothesis on $E_{1}$ and $E_{2}$ give that there is $\delta_{0}>0$ such that $\left|z_{2}-z_{1}\right| \geq \delta_{0}\left|z_{1}\right|$ for all $z_{1} \in E_{1}$ and $z_{2} \in E_{2}$, i.e., $\left|z_{2} / z_{1}-1\right| \geq \delta_{0}$ for all $z_{1} \in E_{1}$ and $z_{2} \in E_{2}$. Consequently, there is $\varepsilon_{0}>0$ such that

$$
E\left(z_{1}, z_{2}\right) \geq \varepsilon_{0}, \quad \text { for all } z_{1} \in E_{1} \text { and } z_{2} \in E_{2} .
$$

Then we have that

$B_{\mathbb{C} \backslash\left\{0, z_{1}\right\}}\left(z_{2}, \varepsilon_{0}\right) \cap B_{\mathbb{C} \backslash\left\{0, z_{2}\right\}}\left(z_{1}, \varepsilon_{0}\right)=\varnothing, \quad$ for all $z_{1} \in E_{1}$ and $z_{2} \in E_{2}$

In the following we will use the notation $B_{\Omega}(A, r):=\cup_{p \in A} B_{\Omega}(p, r)$ for a set $A$ and a positive number $r$.

Let us fix $z_{1} \in E_{1}$. We have that

$$
B_{\left.\mathbb{C} \backslash 0, z_{1}\right\}}\left(E_{2}, \varepsilon_{0}\right):=\cup_{z_{2} \in E_{2}} B_{\mathbb{C} \backslash\left\{0, z_{1}\right\}}\left(z_{2}, \varepsilon_{0}\right)
$$

and

$$
B_{\mathbb{C} \backslash\left\{0, E_{2}\right\}}\left(z_{1}, \varepsilon_{0}\right) \subset \cap_{z_{2} \in E_{2}} B_{\mathbb{C} \backslash\left\{0, z_{2}\right\}}\left(z_{1}, \varepsilon_{0}\right) .
$$

Therefore

$$
B_{\mathbb{C} \backslash\left\{0, z_{1}\right\}}\left(E_{2}, \varepsilon_{0}\right) \cap B_{\mathbb{C} \backslash\left\{0, E_{2}\right\}}\left(z_{1}, \varepsilon_{0}\right)=\varnothing, \quad \text { for all } z_{1} \in E_{1} .
$$

Now, we have that

$$
B_{\mathbb{C} \backslash\left\{0, E_{1}\right\}}\left(E_{2}, \varepsilon_{0}\right) \subset \cap_{z_{1} \in E_{1}} B_{\mathbb{C} \backslash\left\{0, z_{1}\right\}}\left(E_{2}, \varepsilon_{0}\right)
$$

and

$$
B_{\mathbb{C} \backslash\left\{0, E_{2}\right\}}\left(E_{1}, \varepsilon_{0}\right)=\cup_{z_{1} \in E_{1}} B_{\mathbb{C} \backslash\left\{0, E_{2}\right\}}\left(z_{1}, \varepsilon_{0}\right) .
$$

Then

$$
B_{\mathbb{C} \backslash\left\{0, E_{1}\right\}}\left(E_{2}, \varepsilon_{0}\right) \cap B_{\mathbb{C} \backslash\left\{0, E_{2}\right\}}\left(E_{1}, \varepsilon_{0}\right)=\varnothing
$$

REMARK 4. Let $E_{1}, E_{2}$ be closed sets in a domain $\Omega \subset \mathbb{C}$ with $z_{0} \in \partial \Omega$. Let $C_{1}, C_{2}$ be closed sets in $\mathbb{C}$, such that each $C_{j}$ is a finite union of cones with vertex in $z_{0}, E_{j} \subset C_{j}$ and $C_{1} \cap C_{2}=\left\{z_{0}\right\}$. Then

$$
\left|z_{1}-z_{2}\right| \geq \delta_{0}\left|z_{1}-z_{0}\right|, \quad \text { for all } z_{1} \in E_{1}, z_{2} \in E_{2} \text {, }
$$

and therefore, $E_{1}, E_{2}$ are weakly separated in $\Omega$.

In order to prove Theorem 8 we shall state some previous results. 
Lemma 7.1. Let $\mathcal{S}$ be a Riemann surface and $\varepsilon>0$. Let $E_{1}, E_{2}$ be two closed weakly $\varepsilon$-separated subsets in $\mathcal{S}$. Let $\mathcal{S}_{k}=S \backslash E_{k}$ be (connected) hyperbolic Riemann surfaces for $k=1,2$ and let $\mathcal{R}$ be a connected component of $\mathcal{S}_{1} \cap \mathcal{S}_{2}=\mathcal{S} \backslash\left(E_{1} \cup E_{2}\right)$. Then,

$$
b(\mathcal{R}) \geq \frac{1}{2} \tanh ^{2} \varepsilon \min \left\{b\left(\mathcal{S}_{1}\right), b\left(\mathcal{S}_{2}\right)\right\} .
$$

Proof. Let $\varphi \in C_{c}^{\infty}(\mathcal{R})$. Obviously $\varphi \in C_{c}^{\infty}\left(\mathcal{S}_{1}\right) \cap C_{c}^{\infty}\left(\mathcal{S}_{2}\right)$ and

$$
\frac{\iint_{\mathcal{S}_{k}}\|\nabla \varphi\|^{2} d w_{k}}{\iint_{\mathcal{S}_{k}} \varphi^{2} d w_{k}} \geq b\left(\mathcal{S}_{k}\right), \quad k=1,2,
$$

where $d w_{1}$ and $d w_{2}$ denote, respectively, the area element in $\mathcal{S}_{1}$ and $\mathcal{S}_{2}$. Recall that $\|\cdot\|$ and $\nabla$ refer also to the corresponding Poincaré metrics.

Let us consider now the open sets

$$
\begin{aligned}
& E_{1, \varepsilon}=\left\{q \in \mathcal{S}_{2}: d_{\mathcal{S}_{2}}\left(q, E_{1}\right)<2 \varepsilon\right\}, \\
& E_{2, \varepsilon}=\left\{q \in \mathcal{S}_{1}: d_{\mathcal{S}_{1}}\left(q, E_{2}\right)<2 \varepsilon\right\} .
\end{aligned}
$$

By hypothesis we have that $E_{1, \varepsilon} \cap E_{2, \varepsilon}=\varnothing$ and therefore $\left(\mathcal{S} \backslash E_{1, \varepsilon}\right) \cup$ $\left(\mathcal{S} \backslash E_{2, \varepsilon}\right)=\mathcal{S}$. On the other hand, we also have as a consequence of (3.2) that

$$
\iint_{\mathcal{S} \backslash E_{1, \varepsilon}} \varphi^{2} d w \leq \operatorname{cotanh}^{2} \varepsilon \iint_{\mathcal{S} \backslash E_{1, \varepsilon}} \varphi^{2} d w_{2}
$$

and

$$
\iint_{\mathcal{S} \backslash E_{2, \varepsilon}} \varphi^{2} d w \leq \operatorname{cotanh}^{2} \varepsilon \iint_{\mathcal{S} \backslash E_{2, \varepsilon}} \varphi^{2} d w_{1},
$$

where $d w$ is the area element in $\mathcal{R}$.

Therefore, we deduce that

$$
\begin{aligned}
\iint_{\mathcal{R}} \varphi^{2} d w & \leq \iint_{\mathcal{S} \backslash E_{1, \varepsilon}} \varphi^{2} d w+\iint_{\mathcal{S} \backslash E_{2, \varepsilon}} \varphi^{2} d w \\
& \leq \operatorname{cotanh}^{2} \varepsilon\left(\iint_{\mathcal{S} \backslash E_{1, \varepsilon}} \varphi^{2} d w_{2}+\iint_{\mathcal{S} \backslash E_{2, \varepsilon}} \varphi^{2} d w_{1}\right) \\
& \leq \operatorname{cotanh}^{2} \varepsilon\left(\iint_{\mathcal{S}_{2}} \varphi^{2} d w_{2}+\iint_{\mathcal{S}_{1}} \varphi^{2} d w_{1}\right) .
\end{aligned}
$$


Recall that $\iint\|\nabla \varphi\|^{2} d w$ is a conformal invariant, i.e.

$$
\iint_{\mathcal{R}}\|\nabla \varphi\|^{2} d w=\iint_{\mathcal{S}_{1}}\|\nabla \varphi\|^{2} d w_{1}=\iint_{\mathcal{S}_{2}}\|\nabla \varphi\|^{2} d w_{2}
$$

We obtain from (7.1) and (7.2) that

$$
\begin{aligned}
\iint_{\mathcal{R}}\|\nabla \varphi\|^{2} d w & \geq \frac{1}{2} \tanh ^{2} \varepsilon \frac{\iint_{\mathcal{S}_{1}}\|\nabla \varphi\|^{2} d w_{1}+\iint_{\mathcal{S}_{2}}\|\nabla \varphi\|^{2} d w_{2}}{\iint_{\mathcal{R}} \varphi^{2} d w} \varphi^{2} d w_{1}+\iint_{\mathcal{S}_{2}} \varphi^{2} d w_{2} \\
& \geq \frac{1}{2} \tanh ^{2} \varepsilon \min \left\{b\left(\mathcal{S}_{1}\right), b\left(\mathcal{S}_{2}\right)\right\},
\end{aligned}
$$

for every $\varphi \in C_{c}^{\infty}(\mathcal{R})$. This finishes the proof of Lemma 7.1.

As a consequence of this lemma one obtains the following results.

Proposition 2. Let $\mathcal{S}$ be a Riemann surface. Let $E_{1}, E_{2}, \ldots, E_{n}$ be weakly separated closed sets in $\mathcal{S}$ such that $\mathcal{S}_{k}=S \backslash E_{k}(k=1, \ldots, n)$ are (connected) hyperbolic Riemann surfaces and let $\mathcal{R}$ be a connected component of $\cap_{k} \mathcal{S}_{k}=\mathcal{S} \backslash \cup_{k} E_{k}$. Then there exists a positive constant c such that

$$
b(\mathcal{R}) \geq c \min _{k} b\left(\mathcal{S}_{k}\right)
$$

Lemma 7.2. Let $\mathcal{S}$ be a hyperbolic Riemann surface. Let $E_{1}, E_{2}$ be two disjoint closed subsets of $\mathcal{S}$ such that $\mathcal{S}_{k}=S \backslash E_{k}$ are connected surfaces for $k=1,2$, let $\mathcal{R}$ be a connected component of $\mathcal{S}_{1} \cap \mathcal{S}_{2}=\mathcal{S} \backslash\left(E_{1} \cup E_{2}\right)$ and let $4 \varepsilon=d_{\mathcal{S}}\left(E_{1}, E_{2}\right)$. Then,

$$
b(\mathcal{R}) \geq \frac{1}{2} \tanh ^{2} \varepsilon \min \left\{b\left(\mathcal{S}_{1}\right), b\left(\mathcal{S}_{2}\right)\right\} .
$$

Lemma 7.2 is a direct consequence of Lemma 7.1 , since $d_{\mathcal{S}}\left(E_{1}, E_{2}\right)$ $=4 \varepsilon$ implies that $E_{1}, E_{2}$ are weakly $\varepsilon$-separated in $\mathcal{S}$.

Proposition 3. Let $\mathcal{S}$ be a hyperbolic Riemann surface. Let $\left\{E_{k}\right\}_{k=1}^{n}$ be a collection of pairwise disjoint closed subsets of $\mathcal{S}$ such that $\mathcal{S}_{k}=S \backslash E_{k}$ 
$(k=1, \ldots, n)$ are connected surfaces, let $\mathcal{R}$ be a connected component of $\cap_{k} \mathcal{S}_{k}=\mathcal{S} \backslash \cup_{k} E_{k}$ and let $\varepsilon=\min _{j \neq k} d_{\mathcal{S}}\left(E_{j}, E_{k}\right)$. Suppose that $\varepsilon>0$. Then, there exists a positive constant $c$, which only depends on $\varepsilon$ and $n$ (but not on $\mathcal{S}$ ), such that

$$
b(\mathcal{R}) \geq c \min _{k} b\left(\mathcal{S}_{k}\right)
$$

REMARK. Let $\left\{E_{k}\right\}_{k=1}^{n}$ be a collection of pairwise disjoint closed subsets of $\hat{\mathbb{C}}$ such that $\hat{\mathbb{C}} \backslash E_{k}$ is a (connected) hyperbolic plane domain for $k=1, \ldots, n$. Let $\Omega_{0}=\hat{\mathbb{C}} \backslash \cup_{k} E_{k}$. Let also $I$ be a strongly uniformly separated set in $\Omega_{0}$ and let $\Omega=\Omega_{0} \backslash I$. A sufficient condition for $\Omega$ to have HII is that each $\hat{\mathbb{C}} \backslash E_{k}$ has HII.

Definition. Let $\mathcal{S}$ be a hyperbolic Riemann surface and let $\gamma_{1}, \ldots, \gamma_{k}$ be simple closed geodesics in $\mathcal{S}$. We say that $G$ is a quasigeodesic domain in $\mathcal{S}$, relatively to $\gamma_{1}, \ldots, \gamma_{k}$, if $G$ is a domain of finite area in $\mathcal{S}$ and $\partial G$ consists of finitely many simple closed curves $\alpha_{1}, \ldots, \alpha_{r}$, where each $\alpha_{i}$ is either a simple closed geodesic or a finite union of subarcs of simple closed geodesics such that if two arcs meet at a point, one of these arcs is a subarc of some $\gamma_{j}$. We define $\partial_{0} G$ as $\partial_{0} G=\partial G \backslash\left\{\gamma_{1} \cup \cdots \cup \gamma_{k}\right\}$.

Obviously, we can have $\partial_{0} G=\varnothing$.

Quasigeodesic domains appear in a natural way as intersection of geodesics domains: If $G_{1}, G_{2}$ are geodesic domains in $\mathcal{S}$, then $G_{1} \cap G_{2}$ is a quasigeodesic domain relatively to $\partial G_{1}$.

We need to talk about collars of geodesics in any hyperbolic Riemann surface $\mathcal{S}$.

Given a simple closed geodesic $\gamma$ in $\mathcal{S}$, a collar about $\gamma$ is a doubly connected domain on $\mathcal{S}$ bounded by two simple closed curves (the boundary curves of the collar) each point of which has the same distance $d$ from $\gamma$. The distance $d$ is called the width of the collar. A collar about $\gamma$ of area $2 \beta$ is called a $\beta$-collar.

Randol $[\mathrm{R}]$ proved that there exists a collar $C_{\gamma}$ of $\gamma$ with width $d_{0}$, such that

$$
\cosh d_{0} \geq \operatorname{coth} \frac{L_{\mathcal{S}}(\gamma)}{2}, \quad A_{\mathcal{S}}\left(C_{\gamma}\right) \geq 2 L_{\mathcal{S}}(\gamma) \operatorname{cosech} \frac{L_{\mathcal{S}}(\gamma)}{2}
$$

Moreover, if $\gamma^{\prime}$ is a geodesic such that $\gamma \cap \gamma^{\prime}=\varnothing$, we also have that $C_{\gamma} \cap \gamma^{\prime}=\varnothing$. 
Randol $[R]$ states the Collar Lemma under the hypothesis that the surface is compact, but the same proof, without any change, works for any hyperbolic Riemann surface.

Lemma 7.3. Let $\mathcal{S}$ be a hyperbolic Riemann surface satisfying HII and let $\left\{\gamma_{1}, \ldots, \gamma_{k}\right\}$ be a collection of pairwise disjoint simple closed geodesics in $\mathcal{S}$. Then, there exists a positive constant $c$ such that

$$
A_{\mathcal{S}}(G) \leq c L_{\mathcal{S}}\left(\partial_{0} G\right)
$$

for any quasigeodesic domain $G$ in $\mathcal{S}$, relatively to $\gamma_{1}, \ldots, \gamma_{k}$, with $L_{\mathcal{S}}\left(\partial_{0} G\right)>0$.

Proof. By the isoperimetric inequality of $\mathcal{S}$, we only need to check (7.3) for quasigeodesic domains $G$ in $\mathcal{S}$, such that $0<L_{\mathcal{S}}\left(\partial_{0} G\right)<$ $L_{\mathcal{S}}(\partial G)$.

First of all, let us consider the compact sets $C_{t, i}=\{p \in \mathcal{S}$ : $\left.d_{\mathcal{S}}\left(p, \gamma_{i}\right) \leq t\right\}$ for positive $t$ and $i \in\{1, \ldots, k\}$. Given a geodesic $\gamma_{i}$ we choose a positive and a negative side of $\gamma_{i}$, denoted respectively by $\gamma_{i}^{+}$ and $\gamma_{i}^{-}$. We denote by $C_{t, i}^{+}$(respectively $C_{t, i}^{-}$) the set of points in $\mathcal{S}$ which are in some geodesic of length $t$ which starts orthogonally to $\gamma_{i}^{+}$ (respectively $\gamma_{i}^{-}$). Obviously, we have that $C_{t, i}=C_{t, i}^{+} \cup C_{t, i}^{-}$. It can happen that $C_{t, i}^{+} \cap C_{t, i}^{-} \neq \gamma_{i}$ if the Riemann surface $\mathcal{S}$ has positive genus (of course, if $\mathcal{S} \backslash \gamma$ is connected).

Let $G_{t, i}^{+}$(respectively $G_{t, i}^{-}$) be the geodesic domain "corresponding" to $C_{t, i}^{+}$(respectively $C_{t, i}^{-}$): each puncture or boundary curve of $G_{t, i}^{+}$is freely homotopic to a boundary curve of $C_{t, i}^{+}$. Denote by $G_{t, i}$ the union $G_{t, i}=\gamma_{i} \cup G_{t, i}^{+} \cup G_{t, i}^{-}$. If for some $i \in\{1, \ldots, k\}$ we have that $G_{t, i}=\gamma_{i}$ for all positive $t$ (the two boundary curves of $C_{t, i}$ are freely homotopic to $\gamma_{i}$ ), then $k=1$ and $\mathcal{S}$ is a doubly connected domain (an annulus), and (7.3) is true since there are not quasigeodesic domains in $\mathcal{S}$. Therefore we can assume without loss of generality that $G_{t, i}$ is not empty for $t \geq t_{0}$ and $i \in\{1, \ldots, k\}$. Observe that $G_{t, i}^{+}$is non decreasing in $t$. In fact, if $t_{1}<t_{2}$ are such that $A_{\mathcal{S}}\left(G_{t_{1}, i}^{+}\right)<A_{\mathcal{S}}\left(G_{t_{2}, i}^{+}\right)$, the constant curvature -1 and Gauss-Bonnet theorem give $A_{\mathcal{S}}\left(G_{t_{1}, i}^{+}\right)+2 \pi \leq$ $A_{\mathcal{S}}\left(G_{t_{2}, i}^{+}\right)$. The same is true for $G_{t, i}^{-}$.

This implies that for each $i \in\{1, \ldots, k\}$ either there exists a positive number $T_{i}^{+}$such that $G_{t, i}^{+}=G_{T_{i}^{+}, i}^{+}$for all $t \geq T_{i}^{+}$, or $A_{\mathcal{S}}\left(G_{t, i}^{+}\right) \rightarrow \infty$ as $t \rightarrow \infty$. The same is true for $G_{t, i}^{-}$with $T_{i}^{-}$. 
Now, let $G$ be a quasigeodesic domain in $\mathcal{S}$, such that $0<L_{\mathcal{S}}\left(\partial_{0} G\right)$ $<L_{\mathcal{S}}(\partial G)$. Therefore, there exists $j \in\{1, \ldots, k\}$ with $\partial G \cap \gamma_{j} \neq \varnothing$. We consider three possibilities:

Case 1. $A_{\mathcal{S}}(G) \geq 2 h(\mathcal{S}) \ell$, with $\ell:=\sum_{i=1}^{k} L_{\mathcal{S}}\left(\gamma_{i}\right)$. In this case,

$$
2 h(\mathcal{S}) \ell \leq A_{\mathcal{S}}(G) \leq h(\mathcal{S}) L_{\mathcal{S}}(\partial G) \leq h(\mathcal{S})\left(L_{\mathcal{S}}\left(\partial_{0} G\right)+\ell\right)
$$

and we obtain that

$$
\ell \leq L_{\mathcal{S}}\left(\partial_{0} G\right)
$$

Therefore,

$$
A_{\mathcal{S}}(G) \leq 2 h(\mathcal{S}) L_{\mathcal{S}}\left(\partial_{0} G\right) .
$$

Case 2. $A_{\mathcal{S}}(G)<2 h(\mathcal{S}) \ell$.

For each $i \in\{1, \ldots, k\}$, let $t_{i}$ be a positive number verifying the two following conditions:

a) $t_{i} \geq T_{i}^{+}$(if there exists $T_{i}^{+}$) or $A_{\mathcal{S}}\left(G_{t_{i}, i}^{+}\right) \geq 2 h(\mathcal{S}) \ell$,

b) $t_{i} \geq T_{i}^{-}$(if there exists $\left.T_{i}^{-}\right)$or $A_{\mathcal{S}}\left(G_{t_{i}, i}^{-}\right) \geq 2 h(\mathcal{S}) \ell$.

Let $\Omega_{i}$ be the geodesic domain $\Omega_{i}:=G_{t_{i}, i}$. We define the following positive numbers

$$
\begin{gathered}
a:=\min \left\{L_{\mathcal{S}}(\gamma): \gamma \text { simple closed geodesic, } \gamma \subset \cup_{i} \overline{\Omega_{i}}\right\}, \\
b:=\max \left\{L_{\mathcal{S}}(\gamma): \gamma \text { simple closed geodesic, } \gamma \subset \cup_{i=1}^{k}\left\{\gamma_{i} \cup \partial \Omega_{i}\right\}\right\} .
\end{gathered}
$$

Recall that $\partial G \cap \gamma_{j} \neq \varnothing$. This fact, the inequalities, $A_{\mathcal{S}}(G)<2 h(\mathcal{S}) \ell$, $L_{\mathcal{S}}\left(\partial_{0} G\right)>0$, and the definition of $t_{j}$ give that one of the two next possibilities holds:

Case 2.1. There exists a simple closed geodesic $\gamma \subset \overline{\Omega_{j}} \cap \partial_{0} G$. Then

$$
L_{\mathcal{S}}\left(\partial_{0} G\right) \geq L_{\mathcal{S}}(\gamma) \geq a .
$$

Case 2.2. here exists a geodesic arc $\eta$ in $\partial_{0} G$ which meets some simple closed geodesic $\gamma \subset \partial \Omega_{j} \cup\left(\cup_{i=1}^{k} \gamma_{i}\right)$.

Observe that if $G$ is not a geodesic domain we are in this situation; in fact, there is a geodesic arc $\eta$ in $\partial G_{0}$ which meets some $\gamma_{i}$.

Collar Lemma $[\mathrm{R}]$ says that $L_{\mathcal{S}}(\eta) \geq d_{0}$, where $d_{0}$ (the width of the collar $C_{\gamma}$ ) satisfies

$$
\cosh d_{0} \geq \operatorname{coth} \frac{L_{\mathcal{S}}(\gamma)}{2} \geq \operatorname{coth} \frac{b}{2}
$$


and

$$
d_{0} \geq D:=\operatorname{Arg} \cosh \left(\operatorname{coth} \frac{b}{2}\right)
$$

(recall that if a geodesic $\gamma^{\prime}$ does not intersect $\gamma$ then $\gamma^{\prime}$ does not intersect $\left.C_{\gamma}\right)$.

Therefore,

$$
L_{\mathcal{S}}\left(\partial_{0} G\right) \geq L_{\mathcal{S}}(\eta) \geq D .
$$

In both cases $(2.1$ and 2.2$) L_{\mathcal{S}}\left(\partial_{0} G\right) \geq \min \{a, D\}=: c_{0}$. Then

$$
A_{\mathcal{S}}(G) \leq h(\mathcal{S})\left(L_{\mathcal{S}}\left(\partial_{0} G\right)+\ell\right) \leq h(\mathcal{S})\left(L_{\mathcal{S}}\left(\partial_{0} G\right)+\ell \frac{L_{\mathcal{S}}\left(\partial_{0} G\right)}{c_{0}}\right)
$$

and

$$
A_{\mathcal{S}}(G) \leq h(\mathcal{S})\left(1+\frac{\ell}{c_{0}}\right) L_{\mathcal{S}}\left(\partial_{0} G\right)
$$

Obviously, $\ell \geq a \geq c_{0}$ and $1+\ell / c_{0} \geq 2$. Therefore, in any case,

$$
A_{\mathcal{S}}(G) \leq h(\mathcal{S})\left(1+\frac{\ell}{c_{0}}\right) L_{\mathcal{S}}\left(\partial_{0} G\right)
$$

Consequently, Lemma 7.3 is true with

$$
c=h(\mathcal{S})\left(1+\frac{\ell}{c_{0}}\right) .
$$

If $\mathcal{S}$ is a hyperbolic Riemann surface, we have considered (open and connected) subsurfaces $\mathcal{S}_{1} \subset \mathcal{S}$, endowed with its own hyperbolic metric. Of course, $\mathcal{S}_{1}$ is a geodesically complete Riemannian manifold with this metric. In the following we will consider also bordered (connected) Riemann subsurfaces $\mathcal{S}_{2} \subset \mathcal{S}$, endowed with the restriction to $\mathcal{S}_{2}$ of the hyperbolic metric of $\mathcal{S}$. Therefore $\mathcal{S}_{2}$ is not a geodesically complete Riemannian manifold with this metric.

Lemma 7.3 and [FR, Lemma 1.2] have the following consequences.

Corollary 5. Let $\mathcal{S}_{1}, \ldots, \mathcal{S}_{m}$ be hyperbolic Riemann surfaces satisfying HII. For $j=1, \ldots, m$, let $\mathcal{S}_{j}^{0}$ be a bordered subsurface of $\mathcal{S}_{j}$ whose border is a set of $n_{j}\left(1 \leq n_{j}<\infty\right)$ pairwise disjoint simple closed geodesics. Let us assume that we can paste $\mathcal{S}_{1}^{0}, \ldots, \mathcal{S}_{m}^{0}$ along their boundaries, obtaining a complete (without boundary) hyperbolic Riemann surface $\mathcal{R}$ 
(recall that we can join two surfaces identifying two boundary geodesics if and only if they have the same length). Then, $\mathcal{R}$ satisfies HII if and only if there exists $1 \leq j \leq m$ such that $A_{\mathcal{S}_{j}}\left(\mathcal{S}_{j}^{0}\right)=\infty$.

Proof. If $A_{\mathcal{S}_{j}}\left(\mathcal{S}_{j}^{0}\right)$ is finite for $j=1, \ldots, m$, then $\mathcal{R}$ also has finite area (since $A_{\mathcal{R}}\left(\mathcal{S}_{j}^{0}\right)=A_{\mathcal{S}_{j}}\left(\mathcal{S}_{j}^{0}\right)$ ) and therefore, it does not satisfy HII.

Let us assume now that $A_{\mathcal{S}_{1}}\left(\mathcal{S}_{1}^{0}\right)=\infty$. Let $\Lambda$ be the union (for $j=1, \ldots, m)$ of the $n_{j}$ geodesics in the boundary of $\mathcal{S}_{j}^{0}$.

Let $G$ be a geodesic domain in $\mathcal{R}$. If $G$ was already a geodesic domain in some $\mathcal{S}_{j}^{0}$, it satisfies (1.1) with constant

$$
h_{1}=\max \left\{h_{g}\left(\mathcal{S}_{1}\right), \ldots, h_{g}\left(\mathcal{S}_{m}\right)\right\} .
$$

In other case, we consider the sets $G_{j}=G \cap \mathcal{S}_{j}^{0}$, for $j=1, \ldots, m$. Let $\partial_{0} G=\partial G \backslash \Lambda$ and $\partial_{j} G=\partial_{0} G \cap \mathcal{S}_{j}^{0}$, for $j=1, \ldots, m$. Let us consider now the set $J$ of the indices $j \in\{1, \ldots, m\}$ such that $L_{\mathcal{R}}\left(\partial_{j} G\right)>0$.

If $J=\varnothing$ then $\partial G$ is contained in $\Lambda$, and there are only a finite number of such $G$. These domains satisfy (1.1) with a fixed constant $h_{2}$, which only depends on $\mathcal{R}$.

If $j \in J$, then Lemma 7.3 gives that

$$
A_{\mathcal{R}}\left(G_{j}\right) \leq c_{j} L_{\mathcal{R}}\left(\partial_{j} G\right) \leq h_{3} L_{\mathcal{R}}\left(\partial_{j} G\right)
$$

where

$$
h_{3}:=\max \left\{c_{1}, \ldots, c_{m}\right\},
$$

since $A_{\mathcal{R}}\left(G_{j}\right)=A_{\mathcal{S}_{j}}\left(G_{j}\right)$ and $L_{\mathcal{R}}\left(\partial_{j} G\right)=L_{\mathcal{S}_{j}}\left(\partial_{j} G\right)$.

Otherwise, Gauss-Bonnet theorem gives that

$$
\sum_{j \in J} A_{\mathcal{R}}\left(G_{j}\right) \geq 2 \pi
$$

Consequently, (7.4) and (7.5) give that

$$
L_{\mathcal{R}}(\partial G) \geq \sum_{j \in J} L_{\mathcal{R}}\left(\partial_{j} G\right) \geq \frac{1}{h_{3}} \sum_{j \in J} A_{\mathcal{R}}\left(G_{j}\right) \geq \frac{2 \pi}{h_{3}} .
$$

Let

$$
A:=\sum_{A_{\mathcal{R}}\left(S_{j}^{0}\right)<\infty} A_{\mathcal{R}}\left(S_{j}^{0}\right)
$$


As a consequence of (7.4) and (7.6), one deduces that

$$
A_{\mathcal{R}}(G) \leq A+\sum_{j \in J} A_{\mathcal{R}}\left(G_{j}\right) \leq \frac{A h_{3}}{2 \pi} L_{\mathcal{R}}(\partial G)+h_{3} L_{\mathcal{R}}\left(\partial_{0} G\right)
$$

Therefore,

$$
h_{g}(\mathcal{R}) \leq \min \left\{h_{1}, h_{2}, h_{3}\left(1+\frac{A}{2 \pi}\right)\right\} .
$$

Now [FR, Lemma 1.2] finishes the proof of Corollary 5.

Corollary 6. Let $\mathcal{S}$ be a hyperbolic Riemann surface satisfying HII. Let $\gamma_{1}, \ldots, \gamma_{k}$ be pairwise disjoint simple closed geodesics in $\mathcal{S}$. Let $\mathcal{S}_{1}$ be any connected component of $\mathcal{S} \backslash\left\{\gamma_{1} \cup \cdots \cup \gamma_{k}\right\}$ with $A_{\mathcal{S}}\left(\mathcal{S}_{1}\right)=\infty$, and let $\mathcal{S}_{0}$ be the Schottky double of $\mathcal{S}_{1}$. Then, $\mathcal{S}_{0}$ satisfies HII.

The Schottky double of $\mathcal{S}_{1}$ is the union of $\overline{\mathcal{S}_{1}}$ and its "reflection" with respect to $\partial \mathcal{S}_{1}$. See [AS, p. 26] for a precise definition.

This corollary was proved in [Ro1, p. 245-248] with similar arguments that those in Lemma 7.3. However, we need the precise statements of Lemma 7.3 and Corollary 5, which are more general than Corollary 6.

We need some additional results. The first one is well-known (see e.g. $[\mathrm{Be}])$.

Lemma 7.4. Let $\mathcal{S}$ be a hyperbolic Riemann surface with a puncture p. Then, we have that

$$
C_{\mathcal{S}}(p, 1) \cap \gamma=\varnothing
$$

for any simple closed geodesic $\gamma$ in $\mathcal{S}$.

We say that a function $f$ is in the class $C^{k}(F)$, where $1 \leq k \leq \infty$ and $F$ is a closed set, if the derivatives of $f$ up to the order $k$ are continuous in $F$ where we define the derivative of $f$ in a point $z \in F$ as the usual limit when we approach to $z$ by taking points in $F$. We just consider with this purpose closed sets $F$ which are closures of open sets with smooth boundaries.

Lemma 7.5. Let $\mathcal{S}$ be a Riemann surface and let $\mathcal{J}$ be a simply connected domain in $\mathcal{S}$ whose boundary is an analytic simple closed curve 
$\eta$. Given a compact subset $K$ of $\mathcal{J}$, an open subset $V$ of $\mathcal{J}$ and a point $q \in V$, there exists a quasiconformal automorphism $f$ of $\mathcal{S}$ such that $\left.f\right|_{\mathcal{S} \backslash \mathcal{J}}$ is the identity map, $f(K) \subset V$ and $f(q)=q$.

Proof. Let us consider a universal covering map $\pi: \mathbb{D} \longrightarrow \mathcal{S}$. Let $\mathcal{J}_{0}$ be a connected component of $\pi^{-1}(\mathcal{J})$. In what follows by $\pi^{-1}$ we mean the inverse function of $\left.\pi\right|_{\mathcal{J}_{0}}$. Let $F_{1}$ (respectively $F_{2}$ ) be a conformal map of $\hat{\mathbb{C}} \backslash \overline{\mathcal{J}_{0}}$ (respectively $\mathcal{J}_{0}$ ) on $\{z \in \hat{\mathbb{C}}:|z|>1\}$ (respectively $\mathbb{D}$ ). Observe that $F_{1}$ and $F_{2}$ have an analytic extension in a neighbourhood of $\eta_{0}=\partial \mathcal{J}_{0}$ since $\eta_{0}$ and $\partial \mathbb{D}$ are analytic curves. Therefore $h=F_{2} \circ F_{1}^{-1}$ is a homeomorphism of $\partial \mathbb{D}$ on itself which has an analytic extension.

It is well known that in this case there is a quasiconformal automorphism $H$ of $\hat{\mathbb{C}}$ such that $H(\overline{\mathbb{D}})=\overline{\mathbb{D}},\left.H\right|_{\partial \mathbb{D}}=h$ and $H \in C^{\infty}(\overline{\mathbb{D}})$. This fact is a consequence of the Beurling-Ahlfors theorem (see $[\mathrm{BA}]$ or $[\mathrm{A}$, p. 69], where they construct a quasiconformal extension $H_{0}: \hat{\mathbb{C}} \longrightarrow \hat{\mathbb{C}}$ of a quasisymmetric map $h_{0}: \hat{\mathbb{R}} \longrightarrow \hat{\mathbb{R}}$, which preserves the differentiability properties of $h_{0}$ ).

We define a bijection $u$ of $\hat{\mathbb{C}}$ on itself by

$$
u(z):= \begin{cases}F_{2}(z), & z \in \mathcal{J}_{0}, \\ \left(H \circ F_{1}\right)(z), & z \notin \mathcal{J}_{0} .\end{cases}
$$

This function is continuous in $\hat{\mathbb{C}}$ since

$$
\left.H \circ F_{1}\right|_{\eta_{0}}=\left.\left.H\right|_{\partial \mathbb{D}} \circ F_{1}\right|_{\eta_{0}}=\left.h \circ F_{1}\right|_{\eta_{0}}=\left.F_{2} \circ F_{1}^{-1} \circ F_{1}\right|_{\eta_{0}}=\left.F_{2}\right|_{\eta_{0}},
$$

and we have that $u \in C^{\infty}\left(\hat{\mathbb{C}} \backslash \eta_{0}\right) \cap C(\hat{\mathbb{C}})$. The regularity properties of $F_{2}$ and $H \circ F_{1}$ in $\eta_{0}$ gives that the distributional derivatives of $u$ in a neighborhood of $\eta_{0}$ are equal to the classical derivatives (we use the differentiability properties only for this argument). Therefore $u$ is a quasiconformal map on $\hat{\mathbb{C}}$ with the same quasiconformality constant than $H$.

Let $M$ be a Möbius map which fixes $\mathbb{D}$, and such that $M(0)=$ $u\left(\pi^{-1}(q)\right) \in \mathbb{D}$. For any $\alpha>0$, let us consider the following quasiconformal automorphism of $\hat{\mathbb{C}}$

$$
v_{\alpha}(z)= \begin{cases}z, & z \notin \mathbb{D} \\ z|z|^{\alpha-1}, & z \in \mathbb{D} .\end{cases}
$$

Let $f_{\alpha}$ be the following homeomorphism from $\mathcal{S}$ on itself.

$$
f_{\alpha}(p)= \begin{cases}p, & p \notin \mathcal{J}, \\ \left(\pi \circ u^{-1} \circ M \circ v_{\alpha} \circ M^{-1} \circ u \circ \pi^{-1}\right)(p), & p \in \mathcal{J} .\end{cases}
$$


Obviously, $\left.f_{\alpha}\right|_{\mathcal{S} \backslash \mathcal{J}}=\left.\mathrm{id}\right|_{\mathcal{S} \backslash \mathcal{J}}$. Observe that $f_{\alpha}$ is continuous in $\mathcal{S}$, since $\left.v_{\alpha}\right|_{\partial \mathbb{D}}=\left.\mathrm{id}\right|_{\partial \mathbb{D}}$ implies that

$$
\left.\left.\left.\left(\pi \circ u^{-1} \circ M\right)\right|_{\partial \mathbb{D}} \circ v_{\alpha}\right|_{\partial \mathbb{D}} \circ\left(M^{-1} \circ u \circ \pi^{-1}\right)\right|_{\eta}=\left.\mathrm{id}\right|_{\eta}
$$

The same argument used to see that $u$ is a quasiconformal map gives that $f_{\alpha}$ is a quasiconformal automorphism of $\mathcal{S}$ for any $\alpha>0$. Observe that $f_{\alpha}(q)=q$ since $\left(M^{-1} \circ u \circ \pi^{-1}\right)(q)=0$.

For a small $\varepsilon>0$ we have that $\left(\pi \circ u^{-1} \circ M\right)(\{z \in \mathbb{C}:|z| \leq \varepsilon\}) \subset V$ since $\left(\pi \circ u^{-1} \circ M\right)(0)=q \in V$. Given the compact set $K \subset \mathcal{J}$ we can choose $\alpha$ such that $\left(v_{\alpha} \circ M^{-1} \circ u \circ \pi^{-1}\right)(K) \subset\{z \in \mathbb{C}:|z| \leq \varepsilon\}$, since $\left(M^{-1} \circ u \circ \pi^{-1}\right)(K)$ is a compact subset of $\mathbb{D}$.

Therefore we obtain that $f_{\alpha}(K) \subset V$ for this $\alpha$.

Lemma 7.6. Let $w$ be a $C^{1}$ homeomorphism of $\partial \mathbb{D}$ on itself. For each $0<r<1$ there exists a quasiconformal automorphism $f$ of $A=$ $\{r \leq|z| \leq 1\}$ such that $\left.f\right|_{\{|z|=r\}}$ is the identity map, $\left.f\right|_{\partial \mathbb{D}}=w$ and $f \in C^{1}(A)$.

Proof. For each $0<r<1$, let us consider the positive number

$$
a=\frac{1}{2 \pi} \log \frac{1}{r}
$$

and the universal covering map

$$
\pi: B=\{0 \leq \operatorname{Im} z \leq a\} \longrightarrow A, \quad \pi(z)=r e^{-2 \pi i z} .
$$

The map $\pi$ is a periodic function with period 1 and satisfies $\pi(\{z: \operatorname{Im} z=0\})=\{z:|z|=r\}, \quad \pi(\{z: \operatorname{Im} z=a\})=\{z:|z|=1\}$.

Therefore, we only need to prove that if $v$ is a $C^{1}$ homeomorphism of $\{z: \operatorname{Im} z=a\}$ on itself with

$$
v(x+1+i a)=v(x+i a)+1, \quad x \in \mathbb{R},
$$

then there exists a $C^{1}$ quasiconformal automorphism $g$ of $B$ on itself such that

$$
\left.g\right|_{\{\operatorname{Im} z=0\}}=\left.\mathrm{id}\right|_{\{\operatorname{Im} z=0\}},\left.\quad g\right|_{\{\operatorname{Im} z=a\}}=v,
$$


and

$$
g(z+1)=g(z)+1, \quad z \in B .
$$

Such a function $g$ can be constructed explicitly. For example, let us consider

$$
g(x+i y)=x\left(1-\frac{y}{a}\right)+\frac{y}{a} v(x+i a) .
$$

It is clear that $g(z+1)=g(z)+1$ for $z \in B$, that $g$ satisfies the boundary conditions, and that $g$ is a $C^{1}$ homeomorphism from $B$ on itself. It is easy to check that $g$ is a quasiconformal map since it is a $C^{1}$ sense-preserving map and $g(z+1)=g(z)+1$ for $z \in B$.

In order to state the following lemma we need a definition. Recall that any bordered Riemann surface $\mathcal{S}$ with a finitely generated fundamental group may be obtained from a compact Riemann surface of genus $g$ by removing $p$ distinct points (the punctures of $\mathcal{S}$ ), $n$ closed disks (whose boundaries represent the ideal boundaries of $\mathcal{S}$ ) and $m$ open disks (whose boundaries are the border of $\mathcal{S}$ ). The vector $(g, p, n, m)$ is called the quasiconformal type of $\mathcal{S}$. It is well known that there exists a quasiconformal mapping between two bordered Riemann surfaces with the same quasiconformal type.

Lemma 7.7. Let $\mathcal{S}$ be a hyperbolic Riemann surface. Let $\left\{g_{1}, \ldots, g_{N}\right\}$ be a family of pairwise disjoint simple closed curves such that each $g_{i}$ is not homotopic to zero or to a puncture in $\mathcal{S}$ and they are pairwise not homotopic.

Let $S_{1}, \ldots, S_{r}, S_{r+1}, \ldots, S_{k}(1 \leq r \leq k-1)$ be the connected components of $\mathcal{S} \backslash\left(g_{1} \cup \cdots \cup g_{N}\right)$, where $S_{r+1}, \ldots, S_{k}$ are (open) surfaces of finite type. We also require that each $g_{j}$ is contained in the boundary of $\overline{S_{n}}$ and $\overline{S_{\ell}}$ with $n \leq r$ and $\ell>r$.

If $g_{j} \subset \overline{S_{m}}$, let $\gamma_{j}$ be the unique simple closed geodesic in $S_{m}$ freely homotopic to the ideal boundary $g_{j}$.

Let $R_{m}(m=1, \ldots, r)$ be the bordered surface obtained by deleting from $S_{m}$ the open funnel $F_{j}$ bounded by $\gamma_{j}$ and the ideal curve $g_{j}$, for every $\gamma_{j} \subset S_{m}$.

Let $R_{m}(m=r+1, \ldots, k)$ be a bordered surface with the same quasiconformal type than $\overline{S_{m}}$ such that the border of $R_{m}$ is constituted by simple closed geodesics with the following condition: if $g_{j}$ is an ideal boundary curve of $S_{n}$ and $S_{m}(n \leq r)$ and $\eta_{i}$ is a boundary curve of $R_{m}$ corresponding to $g_{j}$, we have that

$$
L_{R_{m}}\left(\eta_{i}\right)=L_{S_{n}}\left(\gamma_{j}\right)
$$


Let $\mathcal{R}$ be a surface obtained by pasting $R_{1}, \ldots, R_{k}$ following the design of $S_{1}, \ldots, S_{k}$ (identifying geodesics of equal length).

Then $\mathcal{S}$ and $\mathcal{R}$ are quasiconformally equivalent.

Proof. Let us fix $m>r$ and let $g_{j_{1}}, \ldots, g_{j_{i}}$ be the boundary curves of $\overline{S_{m}}$. Let us consider $M_{m}=\overline{S_{m}} \cup \overline{F_{j_{1}}} \cup \cdots \cup \overline{F_{j_{i}}} \subset S$.

It is well-known that there is a $C^{1}$ quasiconformal map $f_{m}$ of $R_{m}$ on $M_{m}$, since $R_{m}$ and $M_{m}$ have the same quasiconformal type.

If $\gamma_{j}$ is contained in $S_{n}(n \leq r)$, let us consider a fixed closed collar $C_{j}$ about $\gamma_{j}$ in $S_{n}$ and let $K_{j}$ be the set $K_{j}=C_{j} \cap R_{n}$. The curve $\gamma_{j}$ is contained in the border of $R_{n}$ and $M_{m}$ for some $m>r$.

Lemma 7.6 gives that there exists a $C^{1}$ quasiconformal automorphism $h_{j}$ of $K_{j}$ such that $\left.h_{j}\right|_{\gamma_{j}}=\left.f_{m}\right|_{\gamma_{j}}$ and $\left.h_{j}\right|_{\partial K_{j} \backslash \gamma_{j}}=\left.\mathrm{id}\right|_{\partial K_{j} \backslash \gamma_{j}}$.

Let us consider the homeomorphism $f$ of $\mathcal{R}$ on $\mathcal{S}$ given by $\left.f\right|_{R_{m}}=$ $f_{m}$ for $m>r,\left.f\right|_{K_{j}}=h_{j}$ for $1 \leq j \leq N$, and $f=$ id otherwise.

It is easy to check that $f$ is a quasiconformal map.

We will need the two following well known facts (see for example [C, Theorem 5.1] or [FR, Lemma 4.2]).

Proposition A. Let $\mathcal{S}$ be a Riemann surface and let $I$ and $J$ be closed subsets of $\mathcal{S}$ such that $\mathcal{S} \backslash I$ is a hyperbolic Riemann surface and every connected component of $J$ has a non-empty intersection with I. If $\mathcal{R}$ is a connected component of $\mathcal{S} \backslash(I \cup J)$ then we have that $\delta(\mathcal{R}) \leq \delta(\mathcal{S} \backslash I)$.

Proposition B. Let $\mathcal{S}_{1}, \mathcal{S}_{2}$ be two hyperbolic Riemann surfaces such that $\mathcal{S}_{1} \subset \mathcal{S}_{2}$ and $\Pi_{1}\left(q, \mathcal{S}_{1}\right) \leq \Pi_{1}\left(q, \mathcal{S}_{2}\right)$ for some $q \in \mathcal{S}_{1}$. Then we have that $\delta\left(\mathcal{S}_{1}\right) \leq \delta\left(\mathcal{S}_{2}\right)$.

Observe that Proposition A is a particular case of Proposition B. The proof of this last one is elementary; it is enough to remark that in $\mathcal{S}_{1}$ there are fewer curves and they are longer.

Proposition 4. Let $\mathcal{S}$ be a hyperbolic Riemann surface with infinite area. Let $C_{1}, \ldots, C_{n}$ be pairwise disjoint compact simply connected subsets of $\mathcal{S}$. Then $\mathcal{S}$ satisfies $\mathrm{HII}$ if and only if $\mathcal{S} \backslash\left(C_{1} \cup \cdots \cup C_{n}\right)$ satisfies HII.

REMARK. It is easy to find examples showing that the conclusion of Proposition 4 is not true if some $C_{j}$ is not compact. 
Proof. We can assume without loss of generality that $n=1$. Let $p$ be a point in $C_{1}$. Theorem 1 gives that the statement of Proposition 4 is equivalent to the following one: $\mathcal{S} \backslash\{p\}$ satisfies HII if and only if $\mathcal{S} \backslash C_{1}$ satisfies $\mathrm{HII}$.

This is trivially true if $C_{1}=p$. Therefore, we can assume that $C_{1}$ has infinitely many points.

Let us assume that $\mathcal{S} \backslash\{p\}$ satisfies HII. Observe that $\mathcal{S} \backslash C_{1} \subset \mathcal{S} \backslash$ $\{p\}$ and that the fundamental groups of the two surfaces are isomorphic. Therefore, Proposition B implies that $\mathcal{S} \backslash C_{1}$ satisfies HII.

Let us assume now that $\mathcal{S} \backslash C_{1}$ satisfies HII. Let $\eta_{1}$ be the simple closed geodesic freely homotopic in $\mathcal{S} \backslash C_{1}$ to the ideal boundary $\partial C_{1}$.

Let $F_{1}$ be the open funnel in $\mathcal{S} \backslash C_{1}$ bounded by $\eta_{1}$ and the ideal boundary $\partial C_{1}$, and let $J_{1}$ be the open set $J_{1}=C_{1} \cup F_{1} \subset \mathcal{S}$. Observe that $\partial J_{1}=\eta_{1}$ is an analytic curve.

Let us consider (in $\mathcal{S}$ ) the open set $V=\left(\{p\} \cup C_{\mathcal{S} \backslash\{p\}}(p, 1 / 4)\right) \cap J_{1}$ and the compact set $C_{1}$. Lemma 7.5 gives that there exists a quasiconformal automorphism $f$ of $\mathcal{S}$ such that $C=f\left(C_{1}\right) \subset V,\left.f\right|_{\mathcal{S} \backslash J_{1}}=\left.\mathrm{id}\right|_{\mathcal{S} \backslash J_{1}}$ and $f(p)=p$. Therefore, $f$ is a quasiconformal map of $\mathcal{S} \backslash C_{1}$ on $\mathcal{S} \backslash C$. [FR, Theorem 1] implies that $\mathcal{S} \backslash C$ satisfies HII. We will prove that $\mathcal{S} \backslash\{p\}$ also satisfies HII.

Let $\eta$ be the simple closed geodesic freely homotopic in $\mathcal{S} \backslash C$ to the ideal boundary $\partial C$. Let $F$ be the open funnel in $\mathcal{S} \backslash C$ bounded by $\eta$ and the ideal boundary $\partial C$, and let $J$ be the open set $J=C \cup F \subset \mathcal{S}$.

Let us consider a geodesic domain $G$ in $\mathcal{S} \backslash\{p\}$ and let $G^{\prime}$ be the corresponding geodesic domain in $\mathcal{S} \backslash C$ : each boundary curve of $G$ is freely homotopic in $\mathcal{S} \backslash\{p\}$ to a boundary curve of $G^{\prime}$; if $G$ contains a collar about the puncture $p$, the curve $\eta$ is a boundary curve of $G^{\prime}$ (observe that $\eta$ is freely homotopic to $p$ in $\mathcal{S} \backslash\{p\}$ ).

Gauss-Bonnet theorem gives that

$$
A_{\mathcal{S} \backslash\{p\}}(G)=A_{\mathcal{S} \backslash C}\left(G^{\prime}\right) .
$$

Lemma 7.3 gives that there exists a positive constant $c$, independent of $G$, such that

$$
A_{\mathcal{S} \backslash C}\left(G^{\prime}\right) \leq c L_{\mathcal{S} \backslash C}\left(\partial G^{\prime} \backslash \eta\right),
$$

since $\mathcal{S} \backslash C$ satisfies HII and $\partial G^{\prime} \neq \eta$. We have that $\partial G^{\prime} \neq \eta$ since there are only two domains in $\mathcal{S} \backslash C$ whose boundary is exactly $\eta$ : $F$ and $\mathcal{S} \backslash \bar{F}$, and both have infinite area in $\mathcal{S} \backslash C$. This last fact is a consequence of the hypothesis $A_{\mathcal{S}}(\mathcal{S})=\infty$. 
We have that $\partial G^{\prime} \subset \mathcal{S} \backslash J \subset \mathcal{S} \backslash C$. Lemma 7.4 implies that $\partial G \subset \mathcal{S} \backslash C_{\mathcal{S} \backslash\{p\}}(p, 1 / 2) \subset \mathcal{S} \backslash C$. These facts give that $\partial G^{\prime}$ and $\partial G$ are far from $C$.

Then, (3.1) implies that the hyperbolic metrics of $\mathcal{S} \backslash\{p\}$ and $\mathcal{S} \backslash C$ are comparable in $(\mathcal{S} \backslash J) \cup\left(\mathcal{S} \backslash C_{\mathcal{S} \backslash\{p\}}(p, 1 / 2)\right)$, since $p \in C$.

Therefore, $L_{\mathcal{S} \backslash C}\left(\partial G^{\prime} \backslash \eta\right)$ and $L_{\mathcal{S} \backslash\{p\}}(\partial G)$ are comparable. This fact, (7.7) and (7.8) give that there is a constant $c_{0}>0$, independent of $G$, such that

$$
A_{\mathcal{S} \backslash\{p\}}(G) \leq c_{0} L_{\mathcal{S} \backslash\{p\}}(\partial G),
$$

and then, [FR, Lemma 1.2] gives that $\mathcal{S} \backslash\{p\}$ satisfies HII.

Definition. We will say that a closed and connected subset $C$ of a Riemann surface $\mathcal{S}$ is of finite type if $C$ is a compact simply connected set or, if it has finitely generated fundamental group and $\partial C$ is a union of simple closed curves.

Proposition 5. Let $\mathcal{S}$ be a hyperbolic Riemann surface with infinite area. Let $C_{1}, \ldots, C_{n}$ be pairwise disjoint closed connected subsets of finite type of $\mathcal{S}$. Then, we have the following facts:

a) If $S_{0}$ is a connected component of $\mathcal{S} \backslash\left(C_{1} \cup \cdots \cup C_{n}\right)$ and $\mathcal{S}$ satisfies HII, then $S_{0}$ satisfies HII.

b) If $\mathcal{S} \backslash\left(C_{1} \cup \cdots \cup C_{n}\right)$ is connected and satisfies HII, then $\mathcal{S}$ satisfies HII.

REMARK. It is easy to construct examples showing that $b$ ) is not true if some $C_{j}$ is not of finite type.

Proof. We can assume without loss of generality that $n=1$ and $C_{1}$ is not a simply connected set (by Proposition 4).

Observe that Proposition 5 is trivial if $\mathcal{S}$ is either a simply or a doubly connected surface. Therefore, without loss of generality we can assume that $\mathcal{S}$ is neither a simply nor doubly connected surface.

Let us assume that $\mathcal{S}$ satisfies HII. Let $p$ be a point in $C_{1}$. Theorem 1 gives $\mathcal{S} \backslash\{p\}$ also satisfies HII.

We have that $S_{0} \subset \mathcal{S} \backslash\{p\}$ and the fundamental group of $S_{0}$ is a subgroup of the fundamental group of $\mathcal{S} \backslash\{p\}$. Therefore, Proposition B implies that $S_{0}$ satisfies HII since $\mathcal{S} \backslash\{p\}$ satisfies HII.

Let us assume now that $\mathcal{S} \backslash C_{1}$ satisfies HII. Let $g_{1}, \ldots, g_{N}$ be the simple closed curves in $\partial C_{1}$. 
Without loss of generality we can assume that each $g_{j}$ is not homotopic to zero. In other case, we have that $\mathcal{S} \backslash C_{1}$ is simply connected, since $\mathcal{S} \backslash C_{1}$ is connected and $C_{1}$ is not simply connected. Therefore, $\mathcal{S}$ is of finite type, since $C_{1}$ is of finite type; then $\mathcal{S}$ satisfies HII, since it has infinite area.

Without loss of generality we can assume that each $g_{j}$ is not homotopic to a puncture $p_{j}$ in $\mathcal{S}$. In other case, Theorem 1 allows us to consider the surface $\mathcal{S}_{1}=\mathcal{S} \cup\left\{p_{j}\right\}$ instead of $\mathcal{S}$. Therefore, we would have that $g_{j}$ is homotopic to zero in $\mathcal{S}_{1}$. Using again the last argument we obtain that $\mathcal{S}_{1}$, and consequently $\mathcal{S}$, satisfies HII.

Let us assume now that there exist two different curves $g_{i}, g_{j}$, freely homotopic in $\mathcal{S}$. In this case, there is a doubly connected domain $D$ in $\mathcal{S}$ such that $\partial D=g_{i} \cup g_{j}$. Then we have that $N=2$, since $\mathcal{S} \backslash C_{1}$ and $C_{1}$ are connected. Therefore, we have that either the set $C_{1}$ is equal to $\bar{D}$ or $\mathcal{S} \backslash C_{1}$ is equal to $D$.

The second possibility implies that $\mathcal{S} \backslash C_{1}$ is a doubly connected domain and therefore, $\mathcal{S}$ is of finite type, since $C_{1}$ is of finite type; then $\mathcal{S}$ satisfies HII, since it has infinite area.

If $C_{1}=\bar{D}$, we can take a closed subset $C$ of finite type of $\mathcal{S}$ such that $C_{1} \subset C$ and $C$ is not a doubly connected set (remember that $\mathcal{S}$ is neither a simply nor a doubly connected surface). Proposition B gives that $\mathcal{S} \backslash C$ satisfies HII, since $\mathcal{S} \backslash C_{1}$ satisfies HII.

Therefore, we can assume without loss of generality that there are not two different curves in $\partial C_{1}$ freely homotopic.

Let $\gamma_{1}, \ldots, \gamma_{N}$ be the simple closed geodesics in $\mathcal{S} \backslash C_{1}$ such that $\gamma_{j}$ is freely homotopic to the ideal boundary $g_{j}$.

Then, we can apply to $\mathcal{S}$ the construction of the surface $\mathcal{R}$ of Lemma 7.7, relative to $\left\{g_{1}, \ldots, g_{N}\right\}$ (with $r=1$ and $k=2$ ).

Corollary 5 implies that $\mathcal{R}$ satisfies HII since $\mathcal{S}$ has infinite area and $\mathcal{S} \backslash C_{1}$ satisfies HII. Finally, $\mathcal{S}$ satisfies HII since Lemma 7.7 implies that $\mathcal{R}$ and $\mathcal{S}$ are quasiconformally equivalent.

We can state now the following general version of theorems 9 and 10.

Theorem 7. Let $\mathcal{S}$ be a Riemann surface and let $E$ be a closed subset of $\mathcal{S}$ such that $\mathcal{S} \backslash E$ is a hyperbolic Riemann surface with $A_{\mathcal{S} \backslash E}(\mathcal{S} \backslash E)=$ $\infty$. Then, the following conditions are equivalent:

1) $\mathcal{S} \backslash E$ satisfies $\mathrm{HII}$. 
2) $\mathcal{S}_{0} \backslash E$ satisfies HII, for any subsurface $\mathcal{S}_{0}$ of $\mathcal{S}$ such that $E$ is contained in $\mathcal{S}_{0}, \mathcal{S}_{0} \backslash E$ is connected, and $\mathcal{S} \backslash \mathcal{S}_{0}$ is a finite union of closed sets of finite type.

3) $\mathcal{S}_{0} \backslash E$ satisfies HII, for some subsurface $\mathcal{S}_{0}$ of $\mathcal{S}$ such that $E$ is contained in $\mathcal{S}_{0}, \mathcal{S}_{0} \backslash E$ is connected, and $\mathcal{S} \backslash \mathcal{S}_{0}$ is a finite union of closed sets of finite type.

4) $\mathcal{S} \backslash(E \cup F)$ satisfies HII for any closed subset $F$ of $\mathcal{S}$ verifying: a) $\mathcal{S} \backslash F$ satisfies HII; b) there exists a set $M$, which is a finite union of pairwise disjoint closed sets of finite type, such that $F \subset M$ and $E \cap M=\varnothing$.

5) $\mathcal{S} \backslash(E \cup F)$ satisfies HII for some closed subset $F$ of $\mathcal{S}$ verifying: a) $\mathcal{S} \backslash F$ satisfies HII; b) there exists a set $M$, which is a finite union of pairwise disjoint closed sets of finite type, such that $F \subset M$ and $E \cap M=\varnothing$.

REMARK. If $E$ and $F$ are closed subsets of a Riemann surface $\mathcal{S}$ and there exists a set $M$ which is a finite union of pairwise disjoint closed sets of finite type such that $F \subset M$ and $E \cap M=\varnothing$, then $E$ and $F$ are weakly separated in $\mathcal{S}$.

Proof. Proposition 5 gives that 1), 2) and 3) are equivalent. Lemma 7.1 and the latest remark give that 1) implies 4). Therefore, since 5) follows directly from 4), we only need to prove that 5) implies 3 ). But this is a consequence of propositions B and 5: Proposition B gives that $(\mathcal{S} \backslash E) \backslash M$ satisfies HII and then Proposition 5 gives that $\mathcal{S} \backslash E$ satisfies HII.

Patterson proved in [P1, Theorem 4] a related result for Riemann surfaces $\mathcal{S}$ of finite area and discrete closed subsets $E$.

As a consequence of Theorem 7 we obtain the following result.

Corollary 7. Given a closed subset $E$ of $\hat{\mathbb{C}}$ with infinitely many points, the following conditions are equivalent:

1) $\hat{\mathbb{C}} \backslash$ E satisfies HII.

2) $\Omega \backslash E$ satisfies HII, for any subdomain $\Omega$ of $\hat{\mathbb{C}}$ of finite type such that $E$ is contained in $\Omega$. 
3) $\Omega \backslash E$ satisfies HII, for some subdomain $\Omega$ of $\hat{\mathbb{C}}$ of finite type such that $E$ is contained in $\Omega$.

4) $\hat{\mathbb{C}} \backslash(E \cup F)$ satisfies HII for any closed subset $F$ of $\hat{\mathbb{C}}$ such that $\hat{\mathbb{C}} \backslash F$ satisfies HII and $E \cap F=\varnothing$.

$5) \hat{\mathbb{C}} \backslash(E \cup F)$ satisfies HII for some closed subset $F$ of $\hat{\mathbb{C}}$ such that $\hat{\mathbb{C}} \backslash F$ satisfies HII and $E \cap F=\varnothing$.

Finally, if we apply $n-1$ times Corollary 7 (and Theorem 1), we obtain the following result which was announced at the beginning of this section.

Theorem 8. Let $E_{1}, \ldots, E_{n}$ be pairwise disjoint closed subsets in $\hat{\mathbb{C}}$ with infinitely many points such that $\Omega_{0}=\hat{\mathbb{C}} \backslash \cup_{k} E_{k}$ is connected. Let $I$ be a strongly uniformly separated set in $\Omega_{0}$ and let $\Omega=\Omega_{0} \backslash I$. Then, we have that $\Omega$ satisfies HII if and only if $\hat{\mathbb{C}} \backslash E_{k}$ satisfies HII for $k=1, \ldots, n$.

\section{Isoperimetric inequality, polarization and symmetrization.}

In general, symmetrization arguments are at the heart of isoperimetric inequalities in Riemannian manifolds of constant sectional curvature, which is the case of hyperbolic Riemann surfaces (see e.g. [Ch2, Chapter 6] and the references therein).

On the other hand, the ideas used in the proof of Theorem 4 (see Section 5) can suggest that there is a relation between the HII-property of a hyperbolic plane domain $\Omega$ and this property for its polarization $\Omega_{p}$. A similar question can be proposed for its circular symmetrization $\Omega_{c s}$ (see $[\mathrm{B}]$ or $[\mathrm{H}]$ for the definition and basic background), since polarization and circular symmetrization are very regular processes. Therefore one could expect that some of the following relations would be true:

a) If $\Omega$ satisfies HII, then $\Omega_{p}$ also satisfies HII.

b) If $\Omega_{p}$ satisfies HII, then $\Omega$ also satisfies HII.

c) If $\Omega$ satisfies HII, then $\Omega_{c s}$ also satisfies HII.

d) If $\Omega_{c s}$ satisfies HII, then $\Omega$ also satisfies HII.

In this section we will show that all these conjectures are false even for Denjoy domains. 
1) Let us consider $E=\left\{a_{n}\right\}$ and $F=\left\{b_{n}\right\}$ two increasing sequences of positive numbers converging to 1 such that $E \cap F=\varnothing$. Let $\Omega=\mathbb{C} \backslash((-\infty,-1] \cup[1, \infty) \cup E \cup(-F))$, where $-F=\left\{-b_{n}\right\}$. We have that $\Omega_{p}=\mathbb{C} \backslash((-\infty,-1] \cup[1, \infty) \cup(-E) \cup(-F))$ and $\Omega_{c s}=$ $\mathbb{C} \backslash((-\infty,-1] \cup(-E) \cup(-F))$. Let us assume also that $E$ and $F$ are strongly uniformly separated in $\mathbb{C} \backslash((-\infty,-1] \cup[1, \infty))$ and that $E \cup F$ is not. Theorem 1 gives that $\Omega$ satisfies HII but $\Omega_{p}$ and $\Omega_{c s}$ do not satisfy HII. This example shows that a) and c) are not true.

2) Let us consider $E=\cup_{k=0}^{\infty}\left[1-2^{-2 k}, 1-2^{-2 k-1}\right] \cup\{1\}$ and $F=$ $\cup_{k=0}^{\infty} I_{k} \cup\{-1\}$, where each $I_{k}$ is a closed interval centered in $-1+$ $3 \cdot 2^{-2 k-3}$ and contained in $\left(-1+2^{-2 k-2},-1+2^{-2 k-1}\right)$. Let $\Omega=$ $\hat{\mathbb{C}} \backslash(E \cup F)$. If $\lim _{k \rightarrow \infty} 2^{2 k}\left|I_{k}\right|=0$, one can check that $\Omega$ does not satisfies HII: It is enough to apply Theorem 2 to geodesic domains "surrounding" $I_{n}$ and $I_{n+1}$.

If $-E=\cup_{k=0}^{\infty}\left[-1+2^{-2 k-1},-1+2^{-2 k}\right] \cup\{-1\}$, we have that $\Omega_{c s}=$ $\hat{\mathbb{C}} \backslash((-E) \cup F)$ and $\Omega_{p}=\Omega_{c s} \backslash\{1\}$. The following argument as in the proof of Proposition 6 (see Section 9) gives that $\Omega_{c s}$ satisfies HII: $\hat{\mathbb{C}} \backslash(-E)$ satisfies HII since it is a modulated domain. Let $a_{k}$ be a point in $I_{k}$ for $k \geq 0$. Theorem 1 gives that $\hat{\mathbb{C}} \backslash\left((-E) \cup\left(\cup_{k=0}^{\infty}\left\{a_{k}\right\}\right)\right)$ satisfies HII. Therefore Proposition A implies that $\Omega_{c s}=\hat{\mathbb{C}} \backslash((-E) \cup F)$ satisfies HII. Theorem 1 gives that $\Omega_{p}=\Omega_{c s} \backslash\{1\}$ also satisfies HII. This example shows that b) and $d$ ) are not true.

\section{Geodesic domains.}

One can think that Theorem 5 could be improved by studying only border sets with six points, in the following way.

Let $\Omega$ be a Denjoy domain, let I be the set of isolated points of $\partial \Omega$ and let $\Omega_{0}=\Omega \cup I$. Then, $\Omega$ has HII if and only if $\Omega$ is admissible and there exists a positive constant $c$ such that for any border set of $\partial \Omega_{0}$ with six points, $B=\left\{b_{1}, \ldots, b_{6}\right\}$, we have that

$$
\sum_{j=1}^{3} \Psi_{\Omega_{0}}\left(\left\{b_{2 j-1}, b_{2 j}, b_{2 j+1}, b_{2 j+2}\right\}\right)>c .
$$


This statement seems to be reasonable since if we want to study a border set $B=\left\{b_{1}, \ldots, b_{2 n}\right\}$, we can "divide" it in border sets with six points.

We prove now by an example that this statement is not true.

EXAMPLE. Let $\Omega$ be the Denjoy domain defined as the complement of a dyadic Cantor set, $\Omega=\hat{\mathbb{C}} \backslash K$, where $K$ is constructed as follows.

Let $E_{0}:=[0,1]$ and suppose that $E_{n}$ has been defined and consists of $2^{n}$ closed disjoint subintervals of $E_{0}$, say $J_{j}$, each of them with length $d_{n}=r_{1} \cdots r_{n}$, with

$$
r_{n}:= \begin{cases}\frac{1}{3}, & \text { for odd } n \\ \frac{1}{n+1}, & \text { for even } n .\end{cases}
$$

We divide each subinterval $J_{j}$ in three intervals, obtaining two closed subintervals $J_{j}^{1}$ and $J_{j}^{2}$ (the children of $J_{j}$ ), each of them with length $d_{n+1}=d_{n} r_{n+1}$ and removing the central interval with length $d_{n}-$ $2 d_{n+1}$. If we denote by $E_{n+1}$ the union of the intervals with length $d_{n+1}$, the Cantor set $K$ is defined as $K:=\cap_{n} E_{n}$.

Let us consider an interval $J$ of $E_{n}$ and the unique simple closed geodesic $\gamma_{n}$ which "surrounds" $J$ in $\Omega$.

For odd $n$ we have

$$
L_{\Omega}\left(\gamma_{n}\right) \geq L_{\widehat{\mathbb{C}} \backslash\{0,1 / 3,2 / 3,1\}}(\gamma)
$$

where $\gamma$ is the geodesic in $\hat{\mathbb{C}} \backslash\{0,1 / 3,2 / 3,1\}$ given by $\gamma:=\{\operatorname{Re} z=1 / 2\}$.

We also have

$$
L_{\Omega}\left(\gamma_{n}\right) \leq L_{\mathbb{C} \backslash\{(-\infty,-1 / 3] \cup[0,1 / 3] \cup[2 / 3, \infty)\}}(\eta),
$$

where $\eta$ is the simple closed geodesic in $\mathbb{C} \backslash\{(-\infty,-1 / 3] \cup[0,1 / 3] \cup$ $[2 / 3, \infty)\}$.

If $B:=\{-1 / 3,0,1 / 3,2 / 3\}$, we have that $r(B)=1 / 3$. Therefore Lemma 4.5 gives

$$
\begin{aligned}
L_{\Omega}\left(\gamma_{n}\right) & \leq L_{\mathbb{C} \backslash\{(-\infty,-1 / 3] \cup[0,1 / 3] \cup[2 / 3, \infty)\}}(\eta) \\
& =L_{\mathbb{C} \backslash\{[-1,0] \cup[1 / 3, \infty)\}}(\sigma),
\end{aligned}
$$

where $\sigma$ is the simple closed geodesic in $\mathbb{C} \backslash\{[-1,0] \cup[1 / 3, \infty)\}$. 
For even $n$ we have

$$
\begin{aligned}
L_{\Omega}\left(\gamma_{n}\right) & \leq L_{\mathbb{C} \backslash\left\{\left(-\infty, 2-r_{n}^{-1}\right] \cup[0,1] \cup\left[r_{n}^{-1}-1, \infty\right)\right\}}\left(\eta_{n}\right) \\
& =L_{\mathbb{C} \backslash\{(-\infty, 1-n] \cup[0,1] \cup[n, \infty)\}}\left(\eta_{n}\right),
\end{aligned}
$$

where $\eta_{n}$ is the simple closed geodesic in $\mathbb{C} \backslash\{(-\infty, 1-n] \cup[0,1] \cup[n, \infty)\}$.

If $B_{n}:=\{1-n, 0,1, n\}$, we have that

$$
r\left(B_{n}\right)=\frac{(n-1)^{2}}{2 n-1} \asymp n .
$$

Therefore Lemma 4.5 gives

$$
\begin{aligned}
L_{\Omega}\left(\gamma_{n}\right) & \leq L_{\mathbb{C}\{\{(-\infty, 1-n] \cup[0,1] \cup[n, \infty)\}}\left(\eta_{n}\right) \\
& =L_{\mathbb{C}\left\{\left\{[-1,0] \cup\left[r\left(B_{n}\right), \infty\right)\right\}\right.}\left(\sigma_{n}\right) \\
& \asymp \Phi_{1}\left(r\left(B_{n}\right)\right) \\
& \asymp \frac{1}{\log n}
\end{aligned}
$$

where $\sigma_{n}$ is the simple closed geodesic in $\mathbb{C} \backslash\left\{[-1,0] \cup\left[r\left(B_{n}\right), \infty\right)\right\}$.

We say that a border set $B$ of $\partial \Omega$ is $n$-basic if it has six points and the three simple closed geodesics associated with it surround an interval $J \subset E_{n}$ and their two children $J^{1}, J^{2} \subset E_{n+1}$. We say that a border set $B$ of $\partial \Omega$ is basic if it is $n$-basic for some $n$.

For a $n$-basic border set $B$, we always have (9.1) since one (respectively two) of the three geodesics associated with $B$ verifies (9.2) if $n$ is odd (respectively even).

Inequalities (9.3) and (9.4) give that there is a finite upper bound $l$ for the length of the geodesics associated with any basic border set. Then, Collar Lemma $[R]$ gives that every geodesic which intersects a geodesic $\gamma$ associated with any basic border set has length at least twice the width $w$ of the collar $C_{\gamma}$ and

$$
w \geq \operatorname{Arg} \cosh \left(\operatorname{cotanh}\left(\frac{l}{2}\right)\right)
$$

Therefore, (9.1) is satisfied by every border set $B$ of $\partial \Omega$ with six points, since at least one of the three geodesics associated with $B$ intersects a geodesic associated with a basic border set. 
However, $\Omega$ does not satisfy HII. To see this, let us consider the geodesic domain $G_{k}$ in $\Omega$ bounded by the $2^{2 k}$ geodesics which surround each interval of $E_{2 k}$.

Gauss-Bonnet theorem gives $A_{\Omega}\left(G_{k}\right)=2 \pi\left(2^{2 k}-2\right)$. Inequality (9.4) gives, for some positive constant $c_{0}$,

$$
L_{\Omega}\left(\partial G_{k}\right) \leq c_{0} \frac{2^{2 k}}{\log (2 k)} .
$$

Therefore

$$
\frac{L_{\Omega}\left(\partial G_{k}\right)}{A_{\Omega}\left(G_{k}\right)} \leq \frac{c_{1}}{\log (2 k)} \longrightarrow 0, \quad \text { as } k \longrightarrow \infty
$$

and this fact gives that $\Omega$ does not satisfy HII.

\section{An open problem.}

In this section we want to discuss about the possibility to find a simpler characterization of the HII-property. In fact, we would like to have a result of the following type:

Conjecture. Let $\Omega$ be a Denjoy domain, let $I$ be the set of isolated points of $\partial \Omega \subset \hat{\mathbb{C}}$ and let $\Omega_{0}=\Omega \cup I$. There exists a function $\Phi$, independent of $\Omega$, such that $\Omega$ has HII if and only if $\Omega$ is admissible and there exists a positive constant $c$ such that for any border set of $\partial \Omega_{0}, B=\left\{b_{1}, \ldots, b_{2 n}\right\}$ with $n \geq 3$, we have that

$$
\frac{1}{n} \sum_{j=1}^{n} \Phi\left(r\left(\left\{b_{2 j-1}, b_{2 j}, b_{2 j+1}, b_{2 j+2}\right\}\right)\right)>c .
$$

We can say something about this function $\Phi$, if it exists.

Proposition 6. Let $\Phi$ be a function verifying the following condition:

If a Denjoy domain $\Omega$ has HII then there exists a positive constant c such that for any border set of $\partial \Omega_{0}, B=\left\{b_{1}, \ldots, b_{2 n}\right\}$ with $n \geq 3$, we have that

$$
\frac{1}{n} \sum_{j=1}^{n} \Phi\left(r\left(\left\{b_{2 j-1}, b_{2 j}, b_{2 j+1}, b_{2 j+2}\right\}\right)\right)>c .
$$


Then $\Phi$ must verify

$$
\limsup _{r \rightarrow 0} \frac{\Phi(r)}{\log \left(\frac{1}{r}\right)}>0 .
$$

Proof. Let us consider the following closed subset $E$ of $[0,1]$

$$
E=\cup_{n=0}^{\infty}\left\{\left[2^{-2 n-1}, 2^{-2 n}\right] \cup I_{n}\right\} \cup\{0\},
$$

where $I_{n}$ is the set of $2 n+1$ points $\left\{x_{n, k}\right\}_{k=-n}^{n}$ in $\left(2^{-2 n-2}, 2^{-2 n-1}\right)$, with $x_{n, \pm k}=\left(3 \pm\left(1-2^{-k}\right)\right) 2^{-2 n-3}$, for $k=0,1, \ldots, n$.

Let $I$ be the discrete set $I=\cup_{n=0}^{\infty} I_{n}$. Let $\Omega_{1}, \Omega_{2}$ be the Denjoy domains $\Omega_{1}=\hat{\mathbb{C}} \backslash E$ and $\Omega_{2}=\Omega_{1} \cup I$.

First we will see that $\Omega_{1}$ and $\Omega_{2}$ have HII:

The set $\Omega_{2}$ is modulated and so [FR, Theorem 3] implies that $\Omega_{2}$ has HII.

Therefore, [FR, Theorem 3] gives also that in order to prove that $\Omega_{1}$ has a HII, we only need to check that $I$ is uniformly separated in $\Omega_{2}$ :

The hyperbolic metrics in $\Omega_{2}$ and $\Omega_{2}^{*}=\Omega_{2} \cup\{\infty\}$ are comparable in each euclidean ball of the complex plane. We also have [BP, Corollary 1] that there is a positive constant $c$ such that

$$
\frac{2}{d(x, E \backslash I)} \geq \lambda_{\Omega_{2}^{*}}(x) \geq \frac{c}{d(x, E \backslash I)}, \quad \text { for } x \in[0,1] \cap \Omega_{2} .
$$

These two facts give that

$$
\lambda_{\Omega_{2}}(x) \asymp \frac{1}{d(x, E \backslash I)}, \quad \text { for } x \in[0,1] \cap \Omega_{2} .
$$

Then we have that

$$
\begin{aligned}
d_{\Omega_{2}}\left(x_{n, k}, x_{n, k+1}\right) & \asymp \int_{x_{n, k}}^{x_{n, k+1}} \frac{d x}{2^{-2 n-1}-x} \\
& =\log \frac{2^{-2 n-1}-x_{n, k}}{2^{-2 n-1}-x_{n, k+1}} \\
& =\log 2 .
\end{aligned}
$$


A similar argument gives the same estimate for $d_{\Omega_{2}}\left(x_{n,-k}, x_{n,-k-1}\right)$. This implies that $I$ is uniformly separated in $\Omega_{2}$, and consequently, that $\Omega_{1}$ has HII.

For each point $x_{n, k} \in I$, let us consider the interval $J_{n, k}=\left[a_{n, k}, b_{n, k}\right]$ such that $x_{n, k} \in J_{n, k}$ and $J_{n, k}$ does not meet any interval of the form $\left[2^{-2 m-1}, 2^{-2 m}\right]$ or another $J_{m, l}$. We also choose $a_{n,-n}=x_{n,-n}$ and $b_{n, n}=x_{n, n}$. Let $J=\cup_{n, k} J_{n, k}$ and $\Omega=\Omega_{1} \backslash J$. The length of these intervals $J_{n, k}$ have been chosen so small in such a way that the length of the geodesics $\gamma_{n, k}$ in $\Omega$ which surrounds only $J_{n, k}$ tends to zero as $n \longrightarrow \infty$ (uniformly in $k$ ).

The domain $\Omega$ has HII (in fact $\delta(\Omega) \leq \delta\left(\Omega_{1}\right)<1$ ) as a consequence of Proposition A (see Section 7).

Let us consider now the border set $B_{n}$ in $\Omega$ given by

$$
B_{n}=\left\{2^{-2 n-2}, a_{n,-n}, b_{n, n}, 2^{-2 n-1}\right\} .
$$

We have that

$$
r\left(B_{n}\right)=r\left(\left\{2^{-2 n-2}, x_{n,-n}, x_{n, n}, 2^{-2 n-1}\right\}\right)=\frac{2^{-2 n}}{1-2^{-n}} .
$$

Since $\Omega$ has HII, the property of $\Phi$, with the border set

$$
\left\{2^{-2 n-2}, a_{n,-n}, b_{n,-n}, \ldots, a_{n, 0}, b_{n, 0}, \ldots, a_{n, n}, b_{n, n}, \ldots, 2^{-2 n-1}\right\},
$$

implies

$$
\frac{1}{2 n+2} \Phi\left(\frac{2^{-2 n}}{1-2^{-n}}\right)+o(1)>c, \quad \text { for all } n \in \mathbb{N} .
$$

Then we have

$$
\limsup _{n \rightarrow \infty} \frac{\Phi\left(\frac{2^{-2 n}}{1-2^{-n}}\right)}{\log \frac{1-2^{-n}}{2^{-2 n}}}=\limsup _{n \rightarrow \infty} \frac{\Phi\left(\frac{2^{-2 n}}{1-2^{-n}}\right)}{2 n \log 2} \geq \frac{c}{\log 2} .
$$

This finishes the proof of Proposition 6 .

Proposition 6 implies that the conjecture is not true for any function $\Phi$ satisfying

$$
\limsup _{r \rightarrow 0} \frac{\Phi(r)}{\log \left(\frac{1}{r}\right)}=0 .
$$


In particular it is not true for the function $\Phi_{2}$ in Theorem 2, but it could be true for $\Phi_{1}$.

In any case, if the conjecture would be true for $\Phi=\Phi_{1}$, the proof should be more sophisticated that our arguments, because it is not true that

$$
\Phi_{1}(r(B)) \asymp L_{\Omega}(\gamma(B))
$$

for any border set $B$ of any Denjoy domain $\Omega$ as $r \rightarrow 0$ (if $\Omega=\mathbb{C} \backslash$ $([-1,-1+r] \cup[-r, 0] \cup[r, 2 r] \cup[2, \infty))$ and $B=\{0, r, \infty,-1\}$, then

$$
\begin{gathered}
r=r(B), \\
\Phi_{1}(r) \asymp \log \left(\frac{1}{r}\right)
\end{gathered}
$$

and Theorem 4 gives that $\left.L_{\Omega}(\gamma(B)) \asymp \log \log (1 / r)\right)$.

Acknowledgements. We would like to thank Professor J. L. Fernández for many useful discussions. Also, we would like to thank the referees for their careful reading of the manuscript and for some helpful suggestions.

\section{References.}

[AS] Ahlfors, L. V., Sario, L., Riemann surfaces. Princeton Univ. Press, 1960.

[B] Baernstein II, A., Integral means, univalent functions and circular symmetrization. Acta Math. 133 (1974), 139-169.

[BP] Beardon, A. F., Pommerenke, Ch., The Poincaré metric of a plane domain. J. London Math. Soc. 18 (1978), 475-483.

[Be] Bers, L., An inequality for Riemann Surfaces. Differential Geometry and Complex Analysis. H. E. Rauch Memorial Volume. Springer-Verlag, 1985.

[BA] Beurling, A., Ahlfors, L. V., The boundary correspondence under quasiconformal mappings. Acta Math. 96 (1956), 125-142.

[Br] Brown Flinn, B., Hyperbolic convexity and level sets of analytic functions. Indiana Univ. J. 32 (1983), 831-841.

[C] Carleson, L., On a class of meromorphic functions and its associated exceptional sets. Thesis. Uppsala (1950). 
[Ch1] Chavel, I., Eigenvalues in Riemannian Geometry. Academic Press, 1984.

[Ch2] Chavel, I., Riemannian Geometry: A Modern Introduction. Cambridge Univ. Press, 1993.

[Che] Cheeger, J., A lower bound for the smallest eigenvalue of the Laplacian. In Problems in Analysis, Princeton Univ. Press (1970), 195-199.

[FR] Fernández, J. L., Rodríguez, J. M., The exponent of convergence of Riemann surfaces. Bass Riemann surfaces. Ann. Acad. Sci. Fenn. Series AI 15 (1990), 165-183.

[H] Hayman, W. K., Subharmonic functions (Volume 2). Academic Press, 1989.

[He] Hejhal, D. A., Universal covering maps for variable regions. Math. Z. 137 (1974), 7-20.

[K] Kra, I., Automorphic forms and Kleinian groups. W. A. Benjamin, 1972.

[LV] Lehto, O., Virtanen, K. I., Quasiconformal mappings in the plane. Springer, 1973.

[M] Minda, A reflection principle for the Poincaré metric and applications to Geometric Function Theory. Complex Variables 8 (1987), 129-144.

[N] Nicholls, P. J., The Ergodic Theory of Discrete Groups. Lecture Notes Series 143, Cambridge Univ. Press, 1989.

[P1] Patterson, S. J., The exponent of convergence of Poincaré series. Monatsh. Math. 82 (1976), 297-315.

[P2] Patterson, S. J., Some examples of Fuchsian groups. Proc. London Math. Soc. 39 (1979), 276-298.

[R] Randol, B., Cylinders in Riemann surfaces. Comment. Math. Helv. 54 (1979), 1-5.

[Ro1] Rodríguez, J. M., Isoperimetric inequalities and Dirichlet functions of Riemann surfaces. Pub. Mat. 38 (1994), 243-253.

[Ro2] Rodríguez, J. M., Two remarks on Riemann surfaces. Pub. Mat. 38 (1994), 463-477.

[SN] Sario, L., Nakai, M., Classification theory of Riemann surfaces. Springer, 1970.

[S] Shimizu, H., On discontinuous groups operating on the product of upper half-planes. Ann. of Math. 77 (1963), 33-71.

[So] Solynin, A. Y., Functional inequalities via polarization. Algebra $i$ Analiz. English translation in St. Petersburg Math. J. 8 (1996), 148-185.

[Su] Sullivan, D., Related aspects of positivity in Riemannian Geometry. $J$. Diff. Geom. 25 (1987), 327-351.

[T] Tsuji, M., Potential Theory in Modern Function Theory. Chelsea, 1959. 
[W] Wolontis, V., Properties of conformal invariants. Amer. J. Math. 74 (1952), 587-606.

Recibido: 29 de octubre de 1.997

Revisado: 18 de marzo de 1.998

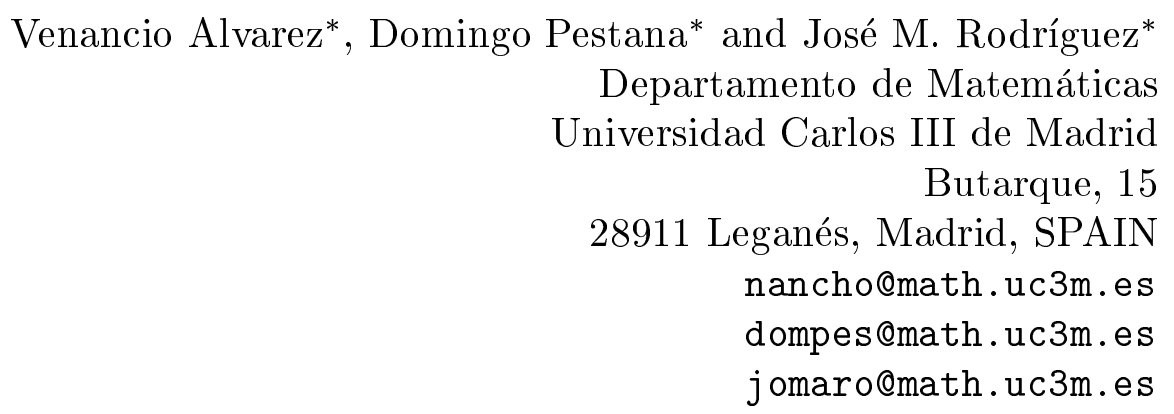

\footnotetext{
* Research partially supported by a grant from DGES (MEC), Spain
} 